\title{
Reconstruction and Local Extensions for Twisted Group Doubles, and Permutation Orbifolds
}

\author{
DAVID E. EVANS \\ School of Mathematics, Cardiff University, \\ Senghennydd Road, Cardiff CF24 4AG, Wales, U.K. \\ e-mail: EvansDE@cf.ac.uk \\ Terry Gannon \\ Department of Mathematics, University of Alberta, \\ Edmonton, Alberta, Canada T6G 2G1 \\ e-mail: tgannon@math.ualberta.ca
}

September 28, 2021

\begin{abstract}
We prove the first nontrivial reconstruction theorem for modular tensor categories: the category associated to any twisted Drinfeld double of any finite group, can be realised as the representation category of a completely rational conformal net. We also show that any twisted double of a solvable group is the category of modules of a completely rational vertex operator algebra. In the process of doing this, we identify the 3-cocycle twist for permutation orbifolds of holomorphic conformal nets: unexpectedly, it can be nontrivial, and depends on the value of the central charge modulo 24 . In addition, we determine the branching coefficients of all possible local (conformal) extensions of any finite group orbifold of holomorphic conformal nets, and identify their modular tensor categories.
\end{abstract}

\section{Contents}

1 Introduction 2

2 Finite group doubles $\quad \mathbf{5}$

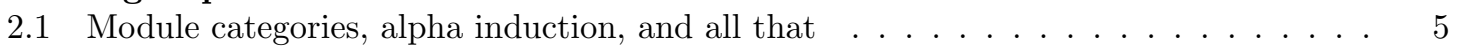

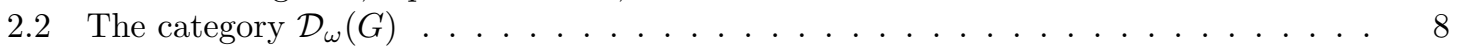

2.3 Bundles over groupoids and twisted group doubles $\ldots \ldots \ldots \ldots \ldots \ldots$

2010 Mathematics Subject Classification. Primary 46L80, 81T40, Secondary 81R05, 81R10, 81R15, 81T05 
3 Results and examples $\quad 14$

3.1 The type 1 module categories of twisted group doubles . . . . . . . . . . . . . 14

3.2 Conformal nets and finite group doubles . . . . . . . . . . . . . . 17

3.3 VOAs and finite group doubles . . . . . . . . . . . . . . . . 20

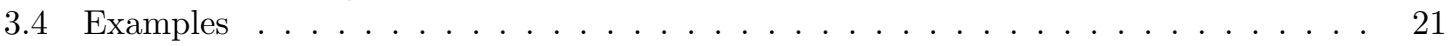

4 Proofs 23

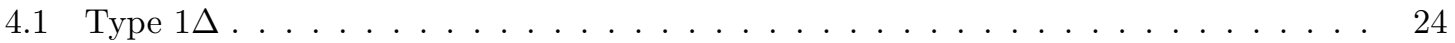

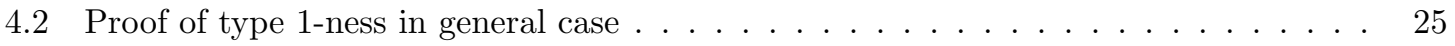

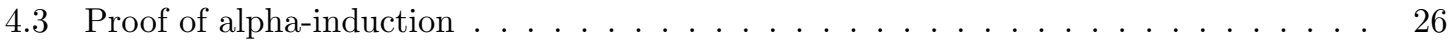

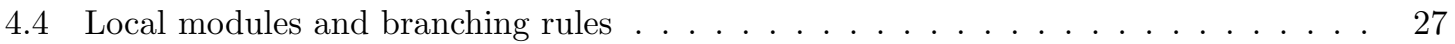

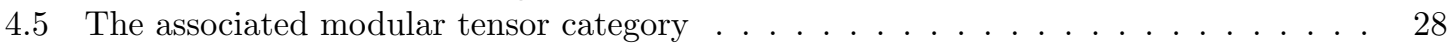

4.6 Proof of Corollary $1 \ldots \ldots \ldots \ldots \ldots$

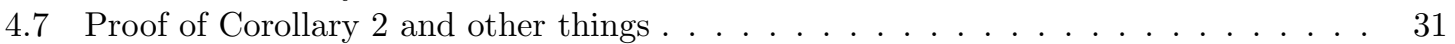

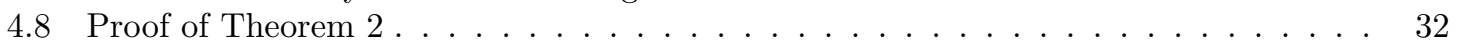

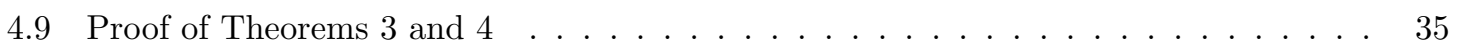

$\begin{array}{ll}\text { References } & 38\end{array}$

\section{Introduction}

The finite-dimensional complex representations of a finite group $G$ form a semi-simple rigid tensor category $\operatorname{Rep}(G)$. It has finitely many simples (one of which is the tensor unit), and the Hom-spaces are finite-dimensional vector spaces. Such categories are called fusion. Moreover, the tensor product is symmetric: the obvious isomorphism $c_{\rho, \phi}: \rho \otimes \phi \rightarrow \phi \otimes \rho$ satisfies $c_{\rho, \phi} \circ c_{\phi, \rho}=i d_{\phi \otimes \rho}$. A version of Tannaka-Krein duality due to Deligne (see e.g. Corollary 9.9.25 of [25]) says that any symmetric fusion category is $\operatorname{Rep}(G)$ (though possibly with the braidings $c_{\rho, \phi}$ twisted by an order 2 element $z$ in the centre of $G$ ) - this is called reconstruction - and $G, z$ are unique up to equivalence.

In section 2.1 we define complete rationality for vertex operator algebras (VOAs) and conformal nets of factors. The modules of completely rational VOAs $\mathcal{V}$ and conformal nets $\mathcal{A}$ also form fusion categories $\operatorname{Mod}(\mathcal{V})$ and $\operatorname{Rep}(\mathcal{A})$ respectively, but now the braiding isomorphisms $c_{M, N}$ are as far as possible from being symmetric. Such categories are called modular tensor categories (MTC). One can ask whether every MTC is braided tensor equivalent to $\operatorname{some} \operatorname{Mod}(\mathcal{V})$ or $\operatorname{Rep}(\mathcal{A})$ (this would be reconstruction), and if so, to what extent this realisation is unique. Little is known about either question, but it has been conjectured that any unitary MTC can be realised as both $\operatorname{Mod}(\mathcal{V})$ and $\operatorname{Rep}(\mathcal{A})$. If so, the realisation will be far from unique, and it is far from clear what plays the role of the central involution $z$ here.

An MTC is a remarkable structure. It carries representations of the modular group $\mathrm{SL}_{2}(\mathbb{Z})$ and indeed every surface mapping class group, gives link invariants for every closed 3-manifold, etc. The trivial MTC is the category $\mathrm{Vec}_{\mathbb{C}}$ of vector spaces, with only one simple object. Reconstruction is easy for $\mathrm{Vec}_{\mathbb{C}}$ : the VOAs and conformal nets with only one simple module are called holomorphic. There are infinitely many 
holomorphic VOAs and conformal nets (e.g. the Monstrous Moonshine VOA), and classifying all of them is hopeless.

The easiest classes of unitary MTC, as well as completely rational VOAs and conformal nets, are associated to even positive-definite lattices $L$. The simples are in natural bijection with the cosets $L^{*} / L$ of the dual lattice by $L$, so self-dual $L$ are holomorphic. All simples in these MTC are invertible (such categories are called pointed); it is not difficult to show that any unitary pointed MTC is the category of modules of both a lattice VOA and lattice conformal net (see e.g. [30]).

The class of unitary MTC we consider in this paper are the twisted Drinfeld doubles $\mathcal{D}_{\omega}(G)$ of finite groups, where $[\omega] \in H^{3}(G ; \mathbb{T})$. Because of their importance and ease of construction, they have a long history, starting with their introduction by Dijkgraaf-Witten [21]. The simple objects are pairs $[g, \chi]$ where $g \in G$ and $\chi$ is a projective irrep of the centraliser $C_{G}(g)$, whose multiplier 2-cocycle comes from $\omega$. These group doubles have a natural interpretation in terms of equivariant $G \times G$ bundles, and this geometric picture plays an important role in our arguments. The importance of twisted group doubles lies in the orbifold construction, one of the fundamental constructions in the theory. If $G$ is a finite group of automorphisms of a VOA or conformal net $\mathcal{A}$, then we define the orbifold $\mathcal{V}^{G}$ or $\mathcal{A}^{G}$ to be the space of points fixed by $G$. When $\mathcal{A}$ is holomorphic, the category $\operatorname{Rep}\left(\mathcal{A}^{G}\right)$ will be $\mathcal{D}_{\omega}(G)$ for some twist $\omega[73],[52]$ (the analogue is conjectured to hold for VOAs).

The easiest and best-studied class of orbifolds are the permutation orbifolds. Let $\mathcal{A}$ (or $\mathcal{V}$ ) be as above, and let $G$ be any subgroup of some symmetric group $S_{k}$. The permutation orbifold of $\mathcal{A}$ by $G$ is $(\mathcal{A} \otimes \cdots \otimes \mathcal{A})^{G}$ where $G$ acts by permuting the $k$ copies of $\mathcal{A}$. When $\mathcal{A}$ is holomorphic, so will be $\mathcal{A} \otimes \cdots \otimes \mathcal{A}$, and the MTC of the permutation orbifold will be $\mathcal{D}_{\omega}(G)$ for some twist $[\omega] \in H^{3}(G ; \mathbb{T})$. It has long been believed (see e.g. Conjecture 6.3 in [63], and section IV(B) in [18]) that the class $[\omega]$ must be trivial, because this construction doesn't see any structure in $\mathcal{A}$. In Theorem 2 we show that $[\omega]$ is not always trivial, though it depends only on the central charge $c$ (a basic numerical invariant) of the net. This shows that the $\mathrm{MTC} \operatorname{Rep}\left(\left(\mathcal{A}^{\otimes k}\right)^{G}\right)$ of permutation orbifolds does not depend only on $G$ and the $\mathrm{MTC} \operatorname{Rep}(\mathcal{A})$ (which here is trivial). The possible nontriviality of $\omega$ can be seen quite easily using characters, as we do at the end of section 3.2. Our proof of Theorem 2 relies on work by Nakaoka [65] on the cohomology of the symmetric group.

We use this to prove reconstruction (Theorem 3) for the twisted group doubles: for any group $G$ and twist $[\omega] \in H^{3}(G ; \mathbb{T}), \mathcal{D}_{\omega}(G)$ is $\operatorname{Rep}\left(\mathcal{A}^{G}\right)$ for some holomorphic net $\mathcal{A}$. This is the first nontrivial class of examples of reconstruction. An important part of our reconstruction proofs uses the 8-term restriction-inflation exact sequence in cohomology.

Extensions of all these results to completely rational VOAs are expected, but the structure theory of VOAs is still behind that of conformal nets. Nevertheless we prove reconstruction for VOAs for arbitrary twists of solvable groups.

A large part of group theory is to understand, and exploit, the subgroups $H$ in a group $G$. The relation between $\operatorname{Mod}(G)$ and $\operatorname{Mod}(H)$ is described by the induction 
and restriction functors. The analogous notion for VOAs and conformal nets is local or conformal extension. However, although the category $\operatorname{Mod}(H)$ is generally smaller the smaller $H$ gets, the category $\operatorname{Mod}(\mathcal{V})$ gets smaller the larger $\mathcal{V}$ gets. In categorical language, extensions $\mathcal{V}^{e}$ of $\mathcal{V}$ correspond to module categories of $\operatorname{Mod}(\mathcal{V})$ of local extension type, also called type 1. Again, induction and restriction functors describe things. For example, the conformal extensions of MTC, VOAs and conformal nets of a lattice $L$, correspond to even lattices $L^{\prime}$ containing $L$ but of the same dimension, so $L \subseteq L^{\prime} \subseteq L^{* *} \subseteq L^{*}$. For example, restriction takes the $\mathcal{A}\left(L^{\prime}\right)$-irrep corresponding to the coset $v+L^{\prime} \in L^{\prime *} / L^{\prime}$ to $v+L^{\prime} \subset L^{*} / L$, i.e. to a sum of $\left|L^{\prime} / L\right|$ irreps for $\mathcal{A}(L)$.

To prove reconstruction we need explicit control of the conformal extensions $\left(\mathcal{A}^{G}\right)^{e}$ of the holomorphic orbifolds $\mathcal{A}^{G}$ (and of $\mathcal{V}^{G}$ ). Equivalently, we need control on all type 1 module categories of any twisted double $\mathcal{D}_{\omega}(G)$. We do that in Theorem 1. We explicitly give the restriction and induction functors (for example the branching coefficients of $\left(\mathcal{A}^{G}\right)^{e}$-modules to $\mathcal{A}$-modules), as well as the MTC of the extension $\left(\mathcal{A}^{G}\right)^{e}$ (it itself is always a twisted group double). These extensions are built from two basic classes: the obvious class corresponds to the orbifold $\mathcal{A}^{K}$ by some subgroup $K \leq G$, and the MTC of the extension is simply $\mathcal{D}_{\omega}(K)$; the other is more difficult to describe, and has MTC $\mathcal{D}_{\omega^{\prime}}(G / N)$ for some normal subgroup $N$ of $G$. Every conformal extension is an extension (possibly trivial) from the first class, followed by one (possibly trivial) from the second. Determining the twist $\omega^{\prime}$ in the second class is quite subtle. Our proof builds on Davydov-Simmons [19] and the thesis [47] of Vaughan Jones. (Incidentally, we describe the remaining module categories of $\mathcal{D}_{\omega}(G)$, e.g. those of type 2 , in [31].)

For example, consider the untwisted case where $G$ is arbitrary but $\operatorname{Rep}\left(\mathcal{A}^{G}\right)$ is braided tensor equivalent to $\mathcal{D}_{1}(G)$. The extreme example of the first class is $K=$ 1: the resulting extension recovers the holomorphic net $\mathcal{A}$ itself, with branching coefficients $\operatorname{Res}(\mathcal{A})=\sum_{\chi} \operatorname{dim} \chi[1, \chi]$. The extreme example of the second class is $N=G$, also a holomorphic net, with branching coefficients $\sum_{g}[g, 1]$. The latter is what is commonly called the orbifold construction, in the physics literature.

The twisted doubles are certainly no less fundamental than the untwisted ones - e.g. Monstrous Moonshine concerns the orbifold of the Moonshine VOA by its full automorphism group, the Monster finite simple group $\mathbb{M}$, and the corresponding $\mathcal{D}_{\omega}(\mathbb{M})$ has order-24 twist $[\omega][45]$. The twists are also nontrivial in Conway and Mathieu Moonshine. The significance of the 3-cocycle $\omega$ is obstruction: the more nontrivial $\omega$ is, the fewer conformal extensions $\mathcal{A}^{G}$ and $\mathcal{V}^{G}$ have, and the fewer (and larger) are their irreps. This is illustrated in Table 1 below.

This paper falls into the sequence of papers [27],[29],[26],[30],[31] on the $K$-theory of loop groups and finite groups. Though it can be read independently, we were led to many of our arguments by this picture, made explicit for finite groups in [26], and we use bundles throughout.

There are two obvious extensions of this work, and we intend to address both in the near future. One is reconstruction for the doubles of other fusion categories associated to finite groups. For example, the weakly group theoretical fusion categories 
conjecturally exhaust all fusion categories with square integer global dimension. We would expect their doubles to be constructed as nested orbifolds starting from a holomorphic net (or VOA). The general case is out of reach at present, but we have completed reconstruction for the Tambara-Yamagami categories [30] (reconstruction of the easier half, that of even rank, has also appeared in [5]). The importance of this is that these can be regarded as the building blocks of doubles of fusion categories such as the Haagerup and other quadratic categories.

The second related extension of this work is to arbitrary finite group orbifolds of conformal nets (or VOAs) whose MTC of representations is pointed (such as the lattice theories). The resulting MTC are also weakly group theoretical. In recent work, Mason and Ng [59] have conjectured the explicit form of these categories, as well as the associated quasi-Hopf algebra. The Verlinde rings have a natural $K$ theoretic interpretation, so seem to be a natural generalisation of the finite group doubles. The challenge is reconstruction for the MTC of [59], as say orbifolds of say lattice theories.

Section 2 reviews the twisted Drinfeld double $\mathcal{D}_{\omega}(G)$ of finite groups, as well as notions of bundles and module categories for finite groups. Section 3 states our results and gives several examples, and section 4 supplies the proofs.

\section{$2 \quad$ Finite group doubles}

\subsection{Module categories, alpha induction, and all that}

For background on modular tensor categories (MTC) and fusion categories, see e.g. [25]. Let $\Phi$ denote the (finite) set of isomorphism classes $\lambda$ of irreducible objects in $\mathcal{C}$; we call these sectors. The Grothendieck ring of $\mathcal{C}$ is called the Verlinde or fusion ring Ver, and has basis $\Phi$.

We occasionally allude to the modular data of an MTC, which is a unitary representation of the modular group $\mathrm{SL}_{2}(\mathbb{Z})$ on the complexification $\mathbb{C} \otimes_{\mathbb{Z}}$ Ver. Since $\mathrm{SL}_{2}(\mathbb{Z})$ is generated by $\left(\begin{array}{cc}0 & -1 \\ 1 & 0\end{array}\right)$ and $\left(\begin{array}{ll}1 & 1 \\ 0 & 1\end{array}\right)$, this representation is uniquely determined by the matrices $S, T \in M_{\Phi \times \Phi}(\mathbb{C})$ corresponding to those generators. $T$ is a diagonal matrix, whereas $S$ determines the tensor product structure constants of Ver through Verlinde's formula.

For the basic theory of vertex operator algebras (VOAs), see e.g. [55]. By a completely rational VOA, we mean $C_{2}$-cofinite, regular, simple, self-dual and of CFTtype. Then the category $\operatorname{Mod}(\mathcal{V})$ of modules of a completely rational VOA is a MTC [42]. For the basic theory of local conformal nets, see e.g. [50]. By a completely rational conformal net, we mean one with the split property and finite $\mu$-index. Then the category $\operatorname{Rep}(\mathcal{A})$ of representations of a completely rational conformal net is a unitary MTC [51]. It is expected that the theory of sufficiently nice (e.g. completely rational and fully unitary) VOAs and their modules should be naturally equivalent to that of sufficiently nice (e.g. completely rational) conformal nets - see [13, 71] for recent progress. 
This paper focuses on the twisted group doubles $\mathcal{D}_{\omega}(G)$, where $G$ is a finite group and $\omega \in Z^{3}(G ; \mathbb{T})$. These unitary MTC are described next subsection. They arise in the theory of VOAs and conformal nets as follows. A completely rational VOA $\mathcal{V}$ or conformal net $\mathcal{A}$ is called holomorphic if its representation theory is trivial, i.e. $\operatorname{Mod}(\mathcal{V}) \operatorname{resp} . \operatorname{Rep}(\mathcal{A})$ is $\operatorname{Vec}_{\mathbb{C}}$. If $G$ is a finite group of automorphisms of a VOA $\mathcal{V}$ or conformal net $\mathcal{A}$ (so by definition $G$ acts faithfully on the underlying spaces $\mathcal{V}$ resp. $\mathcal{H}$ ), by the orbifold $\mathcal{V}^{G}$ resp. $\mathcal{A}^{G}$ we mean the associated VOA resp. conformal net structure on the space of fixed points of all $g \in G$ - see e.g. [55],[73] for details. If $\mathcal{A}$ is completely rational then so is $\mathcal{A}^{G}$ [73]; the analogue is conjectured for VOAs (see e.g. $[23,12,61]$ for recent progress). When $\mathcal{A}$ is holomorphic, the category $\operatorname{Rep}\left(\mathcal{A}^{G}\right)$ will be $\mathcal{D}_{\omega}(G)$ for some twist $\omega[52]$ (the analogue is expected for VOAs but is known only in certain cases).

Any completely rational VOA or conformal net comes with a representation of the Virasoro algebra. In this representation, the (normalised) Virasoro central term $C$ is sent to a multiple of the identity. The central charge $c$ of a conformal net or VOA is that numerical factor. The corresponding MTC determines $c$ only up to mod 8. In particular, the central charge of a holomorphic net or VOA is a multiple of 8. The central charge enters Theorem 2 below.

A conformal or local extension $\mathcal{V}^{e}$ resp. $\mathcal{A}^{e}$ of a completely rational VOA $\mathcal{V}$ or conformal net $\mathcal{A}$ is a completely rational VOA or conformal net containing $\mathcal{V}$ resp. $\mathcal{A}$ but with the same central charge, so that any $\mathcal{V}^{e}$-module or $\mathcal{A}^{e}$-representation restricts to $\operatorname{Mod}(\mathcal{V})$ resp. $\operatorname{Rep}(\mathcal{A})$. These restrictions are called branching rules. Such extensions correspond to commutative symmetric special Frobenius algebras $A$ in the $\operatorname{MTC} \mathcal{C}=\operatorname{Mod}(\mathcal{V})$ or $\operatorname{Rep}(\mathcal{A})$ as follows (for VOAs this is developed in $[53,43,16]$, and the same arguments work for conformal nets). Here, $A$ is the restriction of $\mathcal{V}^{e}$ or $\mathcal{A}^{e}$ to $\mathcal{V}$ resp. $\mathcal{A}$; to be an algebra it must have a multiplication $\mu_{A}$, a morphism $A \otimes A \rightarrow A$; to be commutative this must satisfy $\mu_{A} \circ c_{A, A}=\mu_{A}$ where $c_{A, A}$ is the braiding. For the definition of symmetric special Frobenius (which we don't need), see e.g. [36]. A left $A$-module is a pair $\left(V, \mu_{V}\right)$, where $V \in \mathcal{C}$ and $\mu_{V}: A \otimes V \rightarrow V$ is the $\mathcal{C}$-morphism corresponding to multiplication. These form a fusion category $\operatorname{Mod}_{\mathcal{C}}(A)$, where the tensor product $V \otimes_{A} W$ is some summand of $V \otimes_{\mathcal{C}} W$. Alpha-induction is the functor $\alpha: \mathcal{C} \rightarrow \operatorname{Mod}_{\mathcal{C}}(A)$ defined on objects by $\alpha(V)=A \otimes V$ and $\mu_{\alpha(V)}=\mu_{A} \otimes i d$, and sigma-restriction is the forgetful functor $\operatorname{Res}: \operatorname{Mod}_{\mathcal{C}}(A) \rightarrow \mathcal{C}$ sending $\left(V, \mu_{V}\right)$ to $V$. Alpha-induction is a tensor functor, and sigma-restriction is its adjoint (i.e. Frobenius reciprocity holds between them). The category $\operatorname{Mod}_{\mathcal{C}}(A)$ is not in general braided; the $\mathrm{MTC} \operatorname{Mod}\left(\mathcal{V}^{e}\right)$ or $\operatorname{Rep}\left(\mathcal{A}^{e}\right)$ is identified with the full subcategory $\operatorname{Mod}_{\mathcal{C}}{ }^{l o c}(A)$ of dyslectic or local objects. The simple objects in $\operatorname{Mod}_{\mathcal{C}}{ }^{l o c}(A)$ consist of the simple $\left(V, \mu_{V}\right) \in \operatorname{Mod}_{\mathcal{C}}(A)$ with twist $\theta_{V} \in \mathbb{C} i d$.

More generally, we have the notion of module category [68]. The MTC $\mathcal{C}$ only describes (part of) the chiral data of the rational CFT. On the other hand, much of the data of the full CFT (boundary data, defect lines, correlation functions, etc) is captured by a module category - see e.g. [36]. A module category $\mathcal{M}$ over an MTC $\mathcal{C}$ is a bifunctor $\otimes: \mathcal{C} \times \mathcal{M} \rightarrow \mathcal{M}$ together with compatible associativity 
and unit isomorphisms. These satisfy the usual pentagon and triangle identities, corresponding to different ways to identify $((X \otimes Y) \otimes Z) \otimes M \cong X \otimes(Y \otimes(Z \otimes M))$ and $(X \otimes 1) \otimes M \cong X \otimes M$ for all objects $X, Y, Z$ of $\mathcal{C}$ and $M \in \mathcal{M}$. There are obvious notions of equivalence and direct sums of module categories, and of indecomposable module categories. The Main Theorem of [68] says that each indecomposable module category of $\mathcal{C}$ is the category $\operatorname{Mod}_{\mathcal{C}}(A)$ of right modules in $\mathcal{C}$ of a symmetric special Frobenius algebra $A \in \mathcal{C}$. When $A$ is not commutative, $\operatorname{Mod}_{\mathcal{C}}(A)$ will no longer be a tensor category. For this reason one also considers the $A$ - $A$-bimodules in $\mathcal{C}$. These form a tensor category, called the full system $\mathcal{C}_{\mathcal{M}}^{*}=F u n_{\mathcal{C}}(\mathcal{M}, \mathcal{M})$, a (typically nonbraided) fusion category Morita-equivalent to (i.e. with the same double as) $\mathcal{C}^{o p}$. There are two ways, called $\alpha_{ \pm}(V)$, to make the left-module $A \otimes V$ into a bimodule: we get the right-module structure through either a braiding $c_{A, V}$ or inverse braiding $c_{V, A}^{-1}$. Then both alpha-inductions $\alpha_{ \pm}$are tensor functors.

This categorical picture was abstracted from the older subfactor picture [56, 66, $72,6,7,8,9]$. In that picture, the objects in the various categories are unital *homomorphisms $\rho, \rho^{\prime}: A \rightarrow B$ between type III factors $A, B$. The tensor product is composition and sums can be defined; equivalence classes (called sectors) are denoted $[\lambda]$. Let ${ }_{N} \mathcal{X}_{N}$ be a (braided) system of endomorphisms on a factor $N$ which realises the simple objects of a MTC $\mathcal{C}$, and Hom-spaces in $\mathcal{C}$ are spaces of intertwiners. Let $\iota: N \rightarrow M$ be the inclusion $N \subset M$ of factors, and let $\bar{\iota}: M \rightarrow N$ be its conjugate. We require ${ }_{N} \mathcal{X}_{N}$ to be braided, and the dual canonical endomorphism $\theta=\bar{\iota} \iota$ to be expressible by ${ }_{N} \mathcal{X}_{N}$. Using the braiding or its opposite, we can lift an endomorphism $\lambda \in{ }_{N} \mathcal{X}_{N}$ of $N$ to one of $M$ in two ways, called the alpha-inductions $\alpha_{ \pm}(\lambda)$. The induced systems ${ }_{M} \mathcal{X}_{M}^{ \pm}=\alpha_{ \pm}\left({ }_{N} \mathcal{X}_{N}\right)$ generate the full system ${ }_{M} \mathcal{X}_{M}$. Sigma-restriction is $\bar{\iota} \iota$. By ${ }_{M} \mathcal{X}_{N}$ we mean all irreducibles appearing in any $\lambda \bar{\iota}$ for $\lambda \in{ }_{N} \mathcal{X}_{N}$. The nimrep, precursor to the notion of module category, is the ${ }_{N} \mathcal{X}_{N}$ action on ${ }_{N} \mathcal{X}_{M}$, given by left composition. The algebra $A$ is $\theta$, with the $Q$-system structure providing the Frobenius algebra structure.

Define $\langle\lambda, \mu\rangle=\operatorname{dim} \operatorname{Hom}_{\mathcal{C}}(\lambda, \mu)$. Then the matrix defined by

$$
\mathcal{Z}_{[\lambda],[\mu]}:=\left\langle\alpha_{+}(\lambda), \alpha_{-}(\mu)\right\rangle
$$

is the modular invariant associated to the system, since it commutes with $S$ and $T$. The modular invariant helps describe how the full CFT is built from chiral data.

The case where the Frobenius algebra $A$ is commutative is called type 1 (or extension type); as we know they correspond to local extensions. In this paper we focus on the type 1 module categories of twisted group doubles. The modular invariant of type 1 module categories is block diagonal. A module category is called type 2 (or automorphism type) if its modular invariant is a permutation matrix. Every module category is a combination of two type 1's and a type 2, in the sense we explain next.

Given a full CFT, it is natural to speak of the maximally extended left and right chiral algebras, called the type 1 parents. In the subfactor picture [7], these correspond to intermediate subfactors $N \subset M_{ \pm} \subset M$ so that $N \subset M_{ \pm}$are type 1 , with dual canonical endomorphisms of the form $\left[\bar{\iota}_{+} \iota_{+}\right]=\sum_{\lambda \in \Phi} \mathcal{Z}_{\lambda, 1}[\lambda]$ and 
$\left[\bar{\iota}_{-} \iota_{-}\right]=\sum_{\lambda \in \Phi} \mathcal{Z}_{1, \lambda}[\lambda]$ respectively, coming from the first row and column of $\mathcal{Z}$. In the category language [35], the type 1 parents are called the left and right centre of the corresponding module category or algebra. The type 1 parents have braided tensor equivalent MTC; they can both be canonically identified with a subsystem of the full system ${ }_{M} \mathcal{X}_{M}$ generated by the intersection ${ }_{M} \mathcal{X}_{M}^{+} \cap{ }_{M} \mathcal{X}_{M}^{-}$, called the neutral system ${ }_{M} \mathcal{X}_{M}^{0}$. Write $b_{ \pm}$for the branching rules, written in matrix form, from the type 1 parents ${ }_{M_{ \pm}} \mathcal{X}_{M_{ \pm}}^{0}$ to $\mathcal{C}={ }_{N} \mathcal{X}_{N}$. Then (2.1) becomes in matrix form

$$
\mathcal{Z}=b_{+} \sigma b_{-}^{t},
$$

where $\sigma$ is a permutation matrix corresponding to the composition of the canonical identifications mentioned above. When the module category is type 1 , the intermediate factors satisfy $M_{+}=M_{-}=M$ and $\sigma=i d$ so (2.2) collapses to $\mathcal{Z}=b_{+} b_{+}^{t}$. Since the branching rules are more fundamental, the modular invariant will henceforth be ignored in this paper.

\subsection{The category $\mathcal{D}_{\omega}(G)$}

Throughout this paper, let $\operatorname{Irr}_{c}(G)$ denote the set of all isomorphism classes of projective irreps of $G$ with 2-cocycle multiplier $c$, and $R_{G}$ the character ring of $G$. We write $g^{h}$ for $h^{-1} g h,{ }^{h} g$ for $h g h^{-1}$ and $\mathrm{cl}_{G}(g)$ for the conjugacy class $\left\{g^{h}: h \in G\right\}$. For any subgroup $K \leq G$, write $C_{K}(g)=\left\{k \in K: g^{k}=g\right\}$; e.g. $C_{G}(g)$ is the centraliser. We write $\Delta_{G}$ for the diagonal subgroup $\{(g, g)\}$ of $G^{2}=G \times G$.

Projective representations of finite groups arise naturally in this theory. We assume the reader is familiar with their basic theory. A standard reference is [49]; the paper [14] treats the theory in parallel to that of linear (a.k.a. true) representations, which is also the philosophy we adopt.

Recall the 2- and 3-cocycle conditions for finite group cohomology:

$$
\begin{aligned}
\psi(x, y) \psi(x y, z) & =\psi(y, z) \psi(x, y z), \\
\omega(g, h, k) \omega(g, h k, l) \omega(h, k, l) & =\omega(g h, k, l) \omega(g, h, k l) .
\end{aligned}
$$

For us these always take values in the unit circle $\mathbb{T} \subset \mathbb{C}$. We call $\psi \in Z^{2}(G ; \mathbb{T})$ normalised when all $\psi(g, 1)=\psi(1, h)=1$, and $\omega \in Z^{3}(G ; \mathbb{T})$ normalised when all $\omega(1, h, k)=\omega(g, 1, k)=\omega(g, h, 1)=1$. Given any functions $f: G \rightarrow \mathbb{T}$ and $F: G^{2} \rightarrow$ $\mathbb{T}$, we get a 2-coboundary by $\delta f(g, h)=f(g h) f(g)^{*} f(h)^{*}$ (where as always we denote complex conjugation with ' $*$ ') and a 3-coboundary by $\delta F(g, h, k)=\frac{F(g, h k)}{F(g, h)} \frac{F(h, k)}{F(g h, k)}$; then $H^{i}(G ; \mathbb{T})$ is the group of $i$-cocycles quotient that of the $i$-coboundaries. Given a 3-cocycle $\omega \in Z^{3}(G ; \mathbb{T})$, define $c_{g}^{\omega}=c_{g}$ by

$$
c_{g}\left(h_{1}, h_{2}\right)=\omega\left(g, h_{1}, h_{2}\right) \omega\left(h_{1}, h_{2}, g^{h_{1} h_{2}}\right) \omega\left(h_{1}, g^{h_{1}}, h_{2}\right)^{*}
$$

(a special case of the slant product $H_{m} \times H^{n} \rightarrow H^{n-m}$ ). Then for all $g, h_{1}, h_{2}, h_{3} \in G$, $\omega$ normalised implies $c_{1}\left(h_{1}, h_{2}\right)=c_{g}\left(1, h_{2}\right)=c_{g}\left(h_{1}, 1\right)=1$ and $c_{g}$ satisfies [2]

$$
c_{g}\left(h_{1}, h_{2}\right) c_{g}\left(h_{1} h_{2}, h_{3}\right)=c_{g}\left(h_{1}, h_{2} h_{3}\right) c_{g^{h_{1}}}\left(h_{2}, h_{3}\right)
$$


and therefore is a 2-cocycle for $C_{G}(g)$. Likewise, given a 2-cocycle $\psi$ on $G$, define

$$
\beta_{g}^{\psi}(h)=\beta_{g}(h):=\psi\left(g, h^{g}\right) \psi(h, g)^{*} .
$$

Then the 2-cocycle condition for $\psi$ directly yields

$$
\begin{aligned}
& \beta_{g g^{\prime}}(h)=\beta_{g}(h) \beta_{g^{\prime}}\left(h^{g}\right), \\
& \beta_{g}(h k)=\beta_{g}(h) \beta_{g}(k) \psi(h, k) \psi\left(h^{g}, k^{g}\right)^{*},
\end{aligned}
$$

for all $g, g^{\prime}, h, k \in G$. In particular, for any $g \in G, \beta_{g}$ is a 1-dimensional representation of $C_{G}(g)$. Moreover, when $g$ and $h$ commute,

$$
\beta_{g^{k}}\left(h^{k}\right)=\beta_{g}(h) .
$$

As mentioned earlier, an important class of MTC, called the twisted group double $\mathcal{D}_{\omega}(G)$, is associated to $G$ and a choice of 3-cocycle $\omega \in Z^{3}(G ; \mathbb{T})$. It was introduced by Dijkgraaf-Witten [21], and developed in [20]. It can be defined as the double (or centre) of the fusion category $\operatorname{Vec}_{\omega}(G)$ of $G$-graded vector spaces where the associativity constraint is defined by $\omega$, and is the category of representations of a quasi-triangular quasi-Hopf algebra [58]. The modular data and some initial observations about modular invariants were made in [15]. The relation to subfactors was worked out explicitly in [32], and this led to the classification [69] of module categories for $\mathcal{D}_{\omega}(G)$. This classification is rather abstract, e.g. it is very unclear which of its module categories are type 1, explicitly what are induction and restriction, what are the type 1 parents, etc. As we explain in section 3.1, some clarification of this is made in $[17,19]$. Inspired by [33], one of the authors of this paper (DEE) established the connection of $\mathcal{D}_{\omega}(G)$ and related structures to $K$-theory [26]. This connection underlies this paper, and is reviewed in the next subsection.

Up to braided tensor equivalence, this category $\mathcal{D}_{\omega}(G)$ depends only on the class $[\omega] \in H^{3}(G ; \mathbb{T}$ ) (and of course the isomorphism class of $G$ ). Moreover, if $\alpha$ is some automorphism of $G$, then $\mathcal{D}_{\omega}(G)$ and $\mathcal{D}_{\alpha(\omega)}(G)$ are again equivalent. The characterisation of when $\mathcal{D}_{\omega}(G)$ and $\mathcal{D}_{\omega^{\prime}}\left(G^{\prime}\right)$ are braided tensor equivalent is given in Corollary 1.5 of $[64]$.

Any $\mathcal{D}_{\omega}(G)$ can be realised using systems of endomorphisms as follows. Consider any type $\mathrm{III}_{1}$ factor $N$, and any subgroup of $\operatorname{Out}(N)$ isomorphic to $G$. Lifting that subgroup to $\operatorname{Aut}(N)$ defines a 3-cocycle $\omega$. Then the Drinfeld or quantum double of this system yields the category $\mathcal{D}_{\omega}(G)$. All groups $G$ and classes $[\omega]$ are realised by subgroups of $\operatorname{Out}(N)$ in this way, for some $N-$ e.g. $N$ can be chosen to be hyperfinite [46].

The sectors (equivalence classes of simple objects) in $\mathcal{D}_{\omega}(G)$ are parametrised by pairs $[g, \chi]$ where $g$ is a conjugacy class representative in $G$ and $\chi \in \operatorname{Irr}_{c_{g}}\left(C_{G}(g)\right)$ is a projective character. The unit is $[1, \mathbf{1}]$. We write $\operatorname{Ver}_{\omega}(G)$ for the corresponding Verlinde ring (Grothendieck ring) of $\mathcal{D}_{\omega}(G) \cdot \operatorname{Ver}_{\omega}(G)$ is isomorphic as a ring to the $\omega$-twisted $G$-equivariant $K$-group ${ }^{\omega} K_{G}^{0}(G)$, and though this is crucial to our sequel [31] it plays no role here. 
We often use in section 4 that a group homomorphism $\phi: G \rightarrow H$ gives rise to $K$-theoretic maps $\phi^{*}: \operatorname{Ver}_{\omega^{\phi}}(H) \rightarrow \operatorname{Ver}_{\omega}(G)$ and $\phi_{!}: \operatorname{Ver}_{\omega}(G) \rightarrow \operatorname{Ver}_{\omega^{\phi}}(H)$, namely $[h, \tilde{\chi}] \mapsto \sum_{g}[g, \tilde{\chi} \circ \phi]$ and $[g, \chi] \mapsto\left[\phi(g), \phi_{!}(\chi)\right]$ respectively, where the sum is over all $G$-orbit representatives $g$ with $h \in \phi^{-1}(G . g)$, and $\phi_{!}(\chi)$ is the wrong-way map (adjoint) of $\tilde{\chi} \circ \phi$. This is easiest to see in the bundle picture described next subsection. For example, when $\phi$ is an embedding, $\phi_{!}(\chi)$ is induction.

An important, though still somewhat mysterious, part of the story concerns the modular data. The generators $S, T$ for this $\mathrm{SL}_{2}(\mathbb{Z})$ action is, in the most general case [15],

$$
\begin{aligned}
S_{\left[a_{1}, \chi_{1}\right],\left[a_{2}, \chi_{2}\right]}^{\omega} & =\frac{1}{|G|} \sum_{g_{i} \in \mathrm{cl}\left(a_{i}\right), g_{1} g_{2}=g_{2} g_{1}} \chi_{1}\left(h_{1}\right)^{*} \chi_{2}\left(h_{2}\right)^{*} \frac{c_{g_{1}}\left(k_{1}^{-1}, h_{1}\right) c_{g_{2}}\left(k_{2}^{-1}, h_{2}\right)}{c_{g_{1}}\left(g_{2}, k_{1}^{-1}\right) c_{g_{2}}\left(g_{1}, k_{2}^{-1}\right)}, \\
T_{\left[a_{1}, \chi_{1}\right],\left[a_{2}, \chi_{2}\right]}^{\omega} & =e^{-2 \pi i c / 24} \delta_{\left[a_{1}, \chi_{1}\right],\left[a_{2}, \chi_{2}\right]} \chi_{1}\left(a_{1}\right) / \chi_{1}(1),
\end{aligned}
$$

where $g_{i}=a_{i}^{k_{i}}$, and $h_{1}:={ }^{k_{1}} g_{2} \in C_{G}\left(a_{1}\right), h_{2}:={ }^{k_{2}} g_{1} \in C_{G}\left(a_{2}\right)$, and where $c \in$ $8 \mathbb{Z}$ is the central charge of the corresponding conformal net or VOA. This $\mathrm{SL}_{2}(\mathbb{Z})$ representation is interpreted in [30] using Chern characters within the bundle picture of section 2.3 .

In section 4.8 we need the modular data for the cyclic group $G=\mathbb{Z}_{n}$. Then $H^{3}\left(\mathbb{Z}_{n} ; \mathbb{T}\right) \cong \mathbb{Z}_{n}$, with the following explicit cocycle representatives (see e.g. [41])

$$
\omega_{q}\left(g_{1}, g_{2}, g_{3}\right)=\exp \left(2 \pi i q g_{1}\left[\left(g_{2}+g_{3}\right) / n\right] / n\right),
$$

where $q \in \mathbb{Z}_{n}$ parametrizes the different cohomology classes, and $[x]$ is truncation (the largest integer not greater than $x$ ). The 2-cocycle $c_{a}^{\omega_{q}}(h, g)$ is coboundary for every $q$ and $a$ (since $H^{2}\left(\mathbb{Z}_{n} ; \mathbb{T}\right)=0$ ), so for each $q$ there are $n^{2}$ sectors, which we can parametrise as $\left[a, \chi_{l}\right]$ where $a \in \mathbb{Z}_{n}$ and $\chi_{l}(b)=e^{2 \pi i b l / n}$ for $l \in \mathbb{Z}_{n}$ are the linear characters of $\mathbb{Z}_{n}$. The modular $T$ matrix (2.12) becomes

$$
T_{\left[a, \chi_{l}\right],\left[a, \chi_{l}\right]}^{\omega_{q}}=e^{-2 \pi i c / 24} \exp \left(2 \pi i\left(q a^{2}+n a l\right) / n^{2}\right) .
$$

In our proof of Theorem 2 below, we use the fact that the $T$ matrix alone uniquely determines the twist $\left[\omega_{q}\right]$, for $G=\mathbb{Z}_{2}, \mathbb{Z}_{3}$ and $\mathbb{Z}_{4}$. In particular, write $n_{k}(k=$ $0,1, \ldots, n-1)$ for the number of eigenvalues in $T^{\omega_{q}}$ equal to $e^{2 \pi i k / n^{2}}$, and consider the generating polynomial $P_{q}(x)=\sum_{k} n_{k} x^{k}$ : then (2.14) tells us

- $G=\mathbb{Z}_{2}$ has generating polynomials $P_{0}(x)=x^{2}+3$ and $P_{1}(x)=x^{3}+x+2$;

- $G=\mathbb{Z}_{3}$ has generating polynomials $P_{0}(x)=2 x^{6}+2 x^{3}+5, P_{1}(x)=2 x^{7}+2 x^{4}+2 x+3$, and $P_{2}(x)=2 x^{8}+2 x^{5}+2 x^{2}+3$

- $G=\mathbb{Z}_{4}$ has generating polynomials $P_{0}(x)=2 x^{12}+4 x^{8}+2 x^{4}+8, P_{1}(x)=2 x^{13}+$ $2 x^{12}+2 x^{9}+2 x^{5}+2 x^{4}+2 x+4, P_{2}(x)=2 x^{14}+2 x^{10}+2 x^{8}+2 x^{6}+2 x^{2}+6$, and $P_{3}(x)=2 x^{15}+2 x^{12}+2 x^{11}+2 x^{7}+2 x^{4}+2 x^{3}+4$.

For $G=\mathbb{Z}_{5}$ and beyond, $T^{\omega_{q}}$ no longer determines $\omega_{q}$. However, for any $G=\mathbb{Z}_{n}$, $T^{\omega_{0}}$ is the only $T^{\omega_{q}}$ of order exactly $n$. 
The proof of Theorem 2 also involves 3-cocycles on $G=\mathbb{Z}_{2} \times \mathbb{Z}_{2} \times \mathbb{Z}_{2}=: \mathbb{Z}_{2}^{3}$. The group $H^{3}\left(\mathbb{Z}_{2}^{3} ; \mathbb{T}\right) \cong \mathbb{Z}_{2}^{7}$ is generated by 6 cocycles inflated from quotients isomorphic to either $\mathbb{Z}_{2}$ or $\mathbb{Z}_{2}^{2}$, together with the cocycle

$$
\omega_{i i i}(a, b, c)=(-1)^{a_{1} b_{2} c_{3}} .
$$

For a given multiplier $\beta \in Z^{2}(G ; \mathbb{T})$ of an abelian group $G$, the projective irreps $\chi \in \operatorname{Irr}_{\beta}(G)$ all have the same dimension $d$, and there are precisely $|G| / d^{2}$ of them. For $\omega_{i i i}$, all $1 \neq g \in \mathbb{Z}_{2}^{3}$ have nontrivial $\beta_{g}=\beta_{g}^{\omega_{i i i}} \in Z^{2}\left(\mathbb{Z}_{2}^{3} ; \mathbb{T}\right)$, each having exactly two projective irreps (both with dimension 2). For example, the irreps $\rho_{\epsilon}$ with multiplier $\beta_{(1,1,1)}$ send $(1,0,0) \mapsto\left(\begin{array}{cc}\epsilon & 0 \\ 0 & -\epsilon\end{array}\right),(0,1,0) \mapsto\left(\begin{array}{c}0 \epsilon \\ \epsilon\end{array}\right)$, and $(0,0,1) \mapsto\left(\begin{array}{cc}0 & -\epsilon i \\ \epsilon i & 0\end{array}\right)$, for $\epsilon= \pm 1$, and we see that $T_{\left[(1,1,1), \rho_{\epsilon}\right],\left[(1,1,1), \rho_{\epsilon}\right]}=\epsilon i$. In fact, the MTC $\mathcal{D}_{\omega_{i i i}}\left(\mathbb{Z}_{2}^{3}\right)$ is braided tensor equivalent to $\mathcal{D}_{1}\left(D_{4}\right)$ (see e.g. [38]) so their $S$ and $T$ matrices coincide.

\subsection{Bundles over groupoids and twisted group doubles}

In this subsection we interpret the $\operatorname{MTC} \mathcal{D}_{\omega}(G)$ and its module categories using bundles over groupoids. The classification of module categories for $\mathcal{D}_{\omega}(G)$ is given in Proposition 1 below.

A groupoid is a category whose morphisms have both left and right inverses see for instance the summary in Appendix A of [34]. When a finite group $G$ acts on a set $\mathrm{X}$, we write $X / / G$ for the corresponding (action) groupoid, with objects $x \in X$ and morphisms $g \in \operatorname{Hom}(x, g . x)$. A map (or homomorphism) between groupoids is a functor between the corresponding categories. An untwisted bundle over a groupoid is a functor from the groupoid to the category of finite-dimensional vector spaces; we include twists, which control the projectivity of the groupoid action on the fibres. For example, for $\psi \in Z^{2}(G ; \mathbb{T}), \psi$-twisted bundles on $\mathrm{pt} / / G$ are projective representations of $G$ with multiplier $\psi$.

The groupoids in this paper can be put in the form $\Gamma / / H^{L} \times K^{R}$ where $H$ resp. $K$ are subgroups of a finite group $\Gamma$ which act on $\Gamma$ by left resp. right multiplication. We twist by cocycles $\widetilde{\omega} \in Z^{3}(\Gamma ; \mathbb{T}), \psi_{L} \in Z^{2}(H ; \mathbb{T}), \psi_{R} \in Z^{2}(K ; \mathbb{T})$, and require the restrictions $\left.[\widetilde{\omega}]\right|_{H}=\left.[\widetilde{\omega}]\right|_{K}=[1]$. In this case, the twisted bundles consist of a vector space $V=\oplus_{\gamma \in \Gamma} V_{\gamma}$ (the total space, an $H^{L} \times K^{R}$-bimodule) carrying a projective action of the twisted group rings $\mathbb{C}_{\psi_{L}} H$ and $\mathbb{C}_{\psi_{R}} K$, such that $h\left(V_{\gamma} k\right)=V_{h \gamma k}$ as spaces. These actions satisfy [26]

$$
\begin{array}{rrr}
\left(h_{1} h_{2}\right) v= & \psi_{L}\left(h_{1}, h_{2}\right) \widetilde{\omega}\left(h_{1}, h_{2}, \gamma\right)^{*} h_{1}\left(h_{2} v\right), \\
v\left(k_{1} k_{2}\right)= & \psi_{R}\left(k_{1}, k_{2}\right) \widetilde{\omega}\left(\gamma, k_{1}, k_{2}\right)\left(v k_{1}\right) k_{2}, \\
h(v k)= & \widetilde{\omega}(h, \gamma, k)(h v) k,
\end{array}
$$

for all $h, h_{i} \in H, k, k_{i} \in K, \gamma \in \Gamma$ and $v \in V_{\gamma}$. A morphism $V \rightarrow W$ between bundles is a set of linear maps $V_{\gamma} \rightarrow W_{\gamma}$ between the fibres which commute with the $H \times K$ action. We explain how to multiply bundles shortly.

By a slight abuse of notation, we speak of bundles over $\Gamma / /_{\left(\tilde{\omega}, \psi_{L}, \psi_{R}\right)} H^{L} \times K^{R}$ rather than $\left(\tilde{\omega}, \psi_{L}, \psi_{R}\right)$-twisted bundles over $\Gamma / / H^{L} \times K^{R}$. We write ${ }_{H}^{\psi_{L}} \mathcal{C}(\Gamma)_{K}^{\tilde{\omega}, \psi_{R}}$ for 
the category of bundles over the groupoid $\Gamma / /_{\left(\tilde{\omega}, \psi_{L}, \psi_{R}\right)} H^{L} \times K^{R}$. When a twist $\psi_{i}$ or $\widetilde{\omega}$ is identically 1 , we usually drop it. Any category of the form ${ }_{H}^{\psi} \mathcal{C}(\Gamma)_{H}^{\tilde{\omega}, \psi}$ is fusion using the bundle product given below, provided $\left.[\widetilde{\omega}]\right|_{H \times H \times H}=1$ (see e.g. section 9.7 of [25]). Its double is the $\operatorname{MTC} \mathcal{D}_{\tilde{\omega}}(\Gamma)$. For $\psi=1$, and when $H$ and $\Gamma$ share no nontrivial normal subgroup, the category ${ }_{H}^{\psi} \mathcal{C}(\Gamma)_{H}^{\tilde{\omega}, \psi}$ recovers the $A$ - $A$ system of the group-subgroup subfactor $A=M \rtimes H \subset M \times \Gamma=B$.

The indecomposable bundles of $\Gamma / /_{\left(\tilde{\omega}, \psi_{L}, \psi_{R}\right)} H^{L} \times K^{R}$ can be constructed as follows. Fix any $H^{L} \times K^{R}$ orbit representative $\gamma \in \Gamma$ and a projective irrep $V_{\gamma}$ of the stabiliser $S=\operatorname{Stab}_{H^{L} \times K^{R}}(\gamma)=\{(h, k) \in H \times K: h \gamma=\gamma k\} \cong H \cap^{\gamma} K$ with 2-cocycle we read off from (2.16)-(2.18):

$c\left((h, k),\left(h^{\prime}, k^{\prime}\right)\right)=\psi_{L}\left(h, h^{\prime}\right) \psi_{R}\left(k^{\prime-1}, k^{-1}\right) \widetilde{\omega}\left(h h^{\prime} \gamma, k^{\prime-1}, k^{-1}\right) \widetilde{\omega}\left(h, h^{\prime}, \gamma\right)^{*} \widetilde{\omega}\left(h, h^{\prime} \gamma, k^{\prime-1}\right)^{*}$

The total space (bimodule) of the bundle associated to this pair $\left[\gamma, V_{\gamma}\right]$ is the induced module $\operatorname{Ind}_{S}^{H^{L} \times K^{R}}\left(V_{\gamma}\right)$, and $h V_{\gamma} k$ forms the fibre over $h \gamma k$ for each $h \in H, k \in K$. Direct sums of bundles are defined as usual.

We multiply bundles as follows. Let $G, H, K$ be subgroups of $\Gamma$ on which the class $[\widetilde{\omega}]$ is trivial, and choose $\psi \in Z^{2}(G ; \mathbb{T}), \psi^{\prime} \in Z^{2}(H ; \mathbb{T}), \psi^{\prime \prime} \in Z^{2}(K ; \mathbb{T})$. Consider bundles $[a, \chi] \in{ }_{G}^{\psi} \mathcal{C}(\Gamma)_{H}^{\tilde{\omega}, \psi^{\prime}}$ and $[b, \phi] \in{ }_{H}^{\psi^{\prime}} \mathcal{C}(\Gamma)_{K}^{\tilde{\omega}, \psi^{\prime \prime}}$. Then $[a, \chi] \otimes[b, \phi]$ is the bundle in ${ }_{G}^{\psi} \mathcal{C}(\Gamma)_{K}^{\tilde{\omega}, \psi^{\prime \prime}}$ defined by

$$
[a, \chi] \otimes[b, \phi]=\sum_{h \in G^{a} \cap H \backslash H / H \cap \cap^{b} K}\left[a h b, \operatorname{Ind}_{G^{a h b} \cap H^{b} \cap K}^{G^{a h b} \cap K}\left(\chi^{h b} \cdot \phi\right)\right],
$$

where ${ }^{b} K=b K b^{-1}, G^{a}=a^{-1} G a, \chi^{k}(g)=\chi\left({ }^{k} g\right)$ etc, and Ind is the induction of projective characters. In the special case where the cocycles $\widetilde{\omega}, \psi, \psi^{\prime}, \psi^{\prime \prime}$ are all identically 1 , this formula reduces to equation (2) of [54]; the proof of the more general case (2.20) however follows from the [54] argument.

The bundle product can be expressed in a more coordinate-free manner in terms of bimodules, as follows, using the product on the base $\Gamma$. Let $V, W$ be the total spaces of the two bundles, i.e. the bimodules with an $\widetilde{\omega}$-projective action of the twisted group rings $\mathbb{C}_{\psi} G^{L}$ and $\mathbb{C}_{\psi^{\prime}} H^{R}$, and $\mathbb{C}_{\psi^{\prime}} H^{L}$ and $\mathbb{C}_{\psi^{\prime \prime}} K^{R}$, as in (2.16)-(2.18). The total space of the product bundle is simply the tensor product $V \otimes_{\mathbb{C}_{\psi^{\prime}} H} W$ of bimodules, and the tensor product $v \otimes w$ of $v \in V_{\gamma}$ and $w \in W_{\gamma^{\prime}}$ lies in the fibre above $\gamma \gamma^{\prime}$. This bimodule picture of the bundle product plays a crucial role in our study of alpha-induction in section 4 .

The point is that (see e.g. $[69,26]) \mathcal{D}_{\omega}(G)$ can be identified with the category ${ }_{\Delta_{G}} \mathcal{C}\left(G^{2}\right)_{\Delta_{G}}^{\tilde{\omega}}$, where

$$
\widetilde{\omega}\left(\left(g_{1}, g_{1}^{\prime}\right),\left(g_{2}, g_{2}^{\prime}\right),\left(g_{3}, g_{3}^{\prime}\right)\right)=\omega\left(g_{1}, g_{2}, g_{3}\right) \omega\left(g_{1}^{\prime}, g_{2}^{\prime}, g_{3}^{\prime}\right)^{*}
$$

The sector $[g, \chi]$ corresponds to the bundle $[(g, 1), \chi]$, where we identify the centraliser $C_{G}(g)$ with the stabiliser $\operatorname{Stab}_{\Delta_{G}^{L \times R}}(g, 1)$. The cocycle $(2.19)\left(\right.$ with $\psi_{L}=\psi_{R}=1$ ) collapses with effort to the cocycle (2.5). The braiding in this bundle picture is 
discussed in [26]. The quantum-dimension of the sector $[g, \chi]$, namely $\|\operatorname{cl}(g)\| \chi(1)$, equals as it should the dimension of the total space divided by $|G|$.

The category-theoretic analogue of this geometric picture is module categories (see e.g. [68, 69]). Identify the (fusion) category ${ }_{1} \mathcal{C}(\Gamma)_{1}^{\tilde{\omega}}$ of bundles over the groupoid $\Gamma / / \tilde{\omega} 1$ with the fusion category $\operatorname{Vec}_{\tilde{\omega}}(\Gamma)$ of $\Gamma$-graded vector spaces (the role of $\widetilde{\omega}$ in both categories is to define the associativity morphism for products). Let $H \leq G$ be such that $\left.[\widetilde{\omega}]\right|_{H}=[1]$, and choose any class $[\psi] \in H^{2}(H ; \mathbb{T})$; we can choose the cocycle $\psi$ so that $d \psi=\widetilde{\omega}$. Then the twisted group algebra $\mathbb{C}_{\psi} H$ is an indecomposable algebra over $\operatorname{Vec}_{\tilde{\omega}}(\Gamma)$; as a bundle over $\Gamma / / \tilde{\omega} 1$ it has a copy of $\mathbb{C}$ over each $h \in H$.

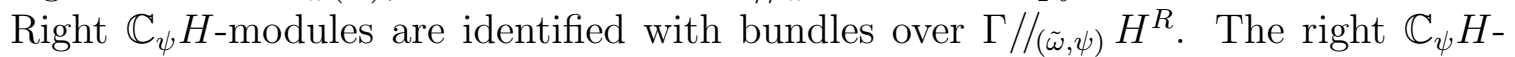
modules form an indecomposable module category ${ }_{1} \mathcal{C}(\Gamma)_{H}^{\tilde{\omega}, \psi}$ over $\operatorname{Vec}_{\tilde{\omega}}(\Gamma)$, and all module categories for $\operatorname{Vec}_{\tilde{\omega}}(\Gamma)$ are of that form (see e.g. Example 2.1 of [69]). The corresponding full system $\mathcal{C}_{\mathcal{M}}^{*}$ for the module category $\mathcal{M}={ }_{1} \mathcal{C}(\Gamma)_{H}^{\tilde{\omega}, \psi}$ is ${ }_{H}^{\psi} \mathcal{C}(\Gamma)_{H}^{\tilde{\omega}, \psi}$.

Continuing this example, we identify $\mathbb{C}_{\psi} H$-bimodules in the category $\operatorname{Vec}_{\tilde{\omega}}(\Gamma)$ with bundles over $\Gamma / /_{(\tilde{\omega}, \psi, \psi)} H^{L} \times H^{R}$, i.e. we identify the fusion categories $\operatorname{Bimod}_{\operatorname{Vec}_{\tilde{\omega}}(\Gamma)}\left(\mathbb{C}_{\psi} H\right)$ and ${ }_{H}^{\psi} \mathcal{C}(\Gamma)_{H}^{\tilde{\omega}, \psi}$. Theorem 3.1 of [69] says the indecomposable module categories over ${ }_{H}^{\psi} \mathcal{C}(\Gamma)_{H}^{\tilde{\omega}, \psi}$ are ${ }_{H}^{\psi} \mathcal{C}(\Gamma)_{H_{1}}^{\tilde{\omega}, \psi_{1}}$, where $H_{1} \leq \Gamma,\left.[\widetilde{\omega}]\right|_{H_{1}}=[1] \in H^{3}\left(H_{1} ; \mathbb{T}\right)$, and $\psi_{1} \in Z^{2}\left(H_{1} ; \mathbb{T}\right)$. Their bundles are $\left(\mathbb{C}_{\psi} H, \mathbb{C}_{\psi_{1}} H_{1}\right)$-bimodules. Two pairs $\left(H_{i}, \psi_{i}\right)$ yield equivalent module categories if they are conjugate, i.e. if there is some $g \in \Gamma$ such that $H_{1}=H_{2}^{g}$ and $\left[\psi_{2}\left(h, h^{\prime}\right)\right]=\left[\psi_{1}\left(h^{g}, h^{\prime g}\right)\right]$.

This leads to Ostrik's characterisation of the module categories of $\mathcal{D}_{\omega}(G)$ :

Proposition 1. ([69], Theorem 3.2) The indecomposable module categories of $\mathcal{D}_{\omega}(G)$ are, up to equivalence, ${ }_{G} \mathcal{C}\left(G^{2}\right)_{H}^{\tilde{\omega}, \psi}$, where $H \leq G \times G,\left.[\tilde{\omega}]\right|_{H}=[1]$, and $[\psi] \in$ $H^{2}(H ; \mathbb{T})$. The module categories ${ }_{\Delta_{G}} \mathcal{C}\left(G^{2}\right)_{H}^{\tilde{\omega}, \psi}$ and ${ }_{\Delta_{G}} \mathcal{C}\left(G^{2}\right)_{H^{\prime}}^{\tilde{\omega}, \psi^{\prime}}$ are equivalent iff $(H,[\psi])$ and $\left(H^{\prime},\left[\psi^{\prime}\right]\right)$ are conjugate.

For example, the identity modular invariant $\mathcal{Z}=I$ corresponds to the choice $H=\Delta_{G}$ and $\psi=1$. More generally, for $H=\Delta_{G}, \psi$ plays the role of discrete torsion, twisting $I$ by the associated cocycles $\beta_{g}^{\psi}$, but for more general $H, \psi$ can act in a much more complicated way, and the general relation between pairs $(H,[\psi])$ and modular invariants $\mathcal{Z}$ is subtle, as we see in Theorem 1.

We can describe the $\mathcal{D}_{\omega}(G)$-module category ${ }_{{ }_{G}} \mathcal{C}\left(G^{2}\right)_{H}^{\tilde{\omega}, \psi}$ corresponding to $(H, \psi)$ in more detail as follows. Let $\left(h_{+}, h_{-}\right) \in H$ act on $g \in G$ on the right by $g \mapsto h_{-}^{-1} g h_{+}$. The indecomposable bundles over $G^{2} / /(\omega, 1, \psi) \Delta_{G}^{L} \times H^{R}$ are parametrised by pairs $[g, \chi]$ for $g$ a representative of an $H$-orbit in $G$, and $\chi$ a projective character of the stabiliser $\left\{\left(h_{+}, h_{-}\right) \in H: h_{-} g=g h_{+}\right\}$in $H$ of $g$. The special bundle, corresponding in the subfactor language to the inclusion $\iota: N \subset M$ of factors, is $\iota=[1, \mathbf{1}]$ while its conjugate $\bar{\iota}$ is the bundle $\left[1, \mathbf{1}^{\prime}\right]$ over $G^{2} / /(\omega, \psi, 1) H^{L} \times \Delta_{G}^{R}$, where $\mathbf{1}, \mathbf{1}^{\prime}$ are certain projective characters on $H \cap \Delta_{G}\left(c_{1}^{\omega}\right.$ equals 1 , but $\psi$ in general contributes a nontrivial 2 -cocycle). Then $\bar{\iota} \iota$ is the algebra $A$ for the module category and the dual canonical endomorphism $\theta$ for the subfactor, and can be computed using (2.20) to be

$$
\theta=A=\sum_{h \in \Delta \cap H \backslash H /(\Delta \cap H)}\left[\Delta h \Delta, \operatorname{Ind}_{\Delta^{h} \cap H \cap \Delta}^{\Delta^{h} \cap \Delta} \mathbf{1} \otimes \mathbf{1}^{\prime}\right] \in{ }_{\Delta} \mathcal{C}\left(G^{2}\right)_{\Delta}^{\omega},
$$


where we write $\Delta$ for $\Delta_{G}$, as in [26] in the untwisted case. The corresponding full system ${ }_{M} \mathcal{X}_{M}$ is ${ }_{H}^{\psi} \mathcal{C}\left(G^{2}\right)_{H}^{\tilde{\omega}, \psi}$ [26]. Alpha-induction, sigma-restriction, the product in ${ }_{M} \mathcal{X}_{M}$, the $\operatorname{Ver}(G)$-module structure of ${ }_{M} \mathcal{X}_{N}$, etc are nontrivially modified by $\psi$.

\section{Results and examples}

\subsection{The type 1 module categories of twisted group doubles}

Let $G$ be finite and $\omega \in Z^{3}(G ; \mathbb{T})$. Recall the classification (Proposition 1) of module categories for the twisted group double $\mathcal{D}_{\omega}(G)$ in terms of pairs $(H, \psi)$. In this subsection we give our first result, Theorem 1 , which is our answer to the following question of Ostrik [69]:

Problem. Which pairs $(H, \psi)$ in Proposition 1 correspond to type 1 (i.e. extensiontype) module categories?

Ostrik also asked to determine explicitly the modular invariant $\mathcal{Z}$ associated to each pair $(H, \psi)$ (not just the type 1 ones). We address that in [31], which gives a remarkably simple and conceptual $K K$-theory description of the module categories for finite group doubles. As we show in [30], this $K K$-theory description extends to the MTC associated to lattices and loop groups.

Alpha-induction and the modular invariant in the case where $\omega=1$ and $\psi=1$, with $\Delta \leq H$, was handled in [26], and the modular invariant, full system, nimrep etc for any $H$ where $\omega=1$ and $\psi=1$ was handled in [29]. For arbitrary $\omega$, DavydovSimmons [19] in Theorem 3.15 identify which pairs $(H, \psi)$ are type 1 (for $\omega=1$, this was done in Theorem 3.5.1 of [17]), and the MTC of local modules is given in Theorem 3.16; Theorem 5.8 gives the modular invariant matrix, though in the basis of commuting pairs $(g, h) \in G^{2}$ rather than the natural basis of sectors $[g, \chi]$ (for $\omega=1$, this was done in Theorem 3.5.3 of [17]). (This commuting pairs basis simplifies many calculations because the modular data $(2.11),(2.12)$ become generalised permutation matrices [2], but e.g. integrality is lost.) To our knowledge, none of alpha-induction, the full system, nor sigma-restriction is given in [19, 17].

Our expressions for the modular invariants are considerably simpler than those in [19]. Their expression for the modular invariant associated to $(H, \psi)$ takes the complicated form

$$
\mathcal{Z}_{\left[f, f^{\prime}\right],\left[g, g^{\prime}\right]}=\sum_{\left(y, y^{\prime}\right) \in Y} \frac{c^{f}\left(g^{y}, y^{-1}\right) c^{f^{\prime}}\left(g^{\prime y^{\prime}}, y^{\prime-1}\right)^{*}}{c^{f}\left(y^{-1}, g\right) c^{f^{\prime}}\left(y^{\prime-1}, g^{\prime}\right)^{*}} \frac{\psi\left(\left(f^{y}, f^{\prime y^{\prime}}\right),\left(g^{y}, g^{\prime y^{\prime}}\right)\right)}{\psi\left(\left(g^{y}, g^{\prime y^{\prime}}\right),\left(f^{y}, f^{\prime y^{\prime}}\right)\right)},
$$

for $c^{f}(a, b)=\omega(a, b, f)^{*} \omega\left(a,{ }^{b} f, b\right) \omega\left(f^{a b}, a, b\right)$, where $Y$ is a set of coset representatives for $\left\{\left(y, y^{\prime}\right) \in G^{2} \mid\left(f^{y}, f^{\prime} y^{\prime}\right) \in H\right\} / H$. Equation (3.1) obscures all the structure present. For one thing, since this is expressed in the wrong basis, the matrix entries of (3.1) aren't in $\mathbb{Z}_{\geq 0}$. Also, for type 1 module categories, sigma-restriction of local modules determines the modular invariant $\mathcal{Z}=b b^{t}$ (when it is not type 1 , this becomes $\mathcal{Z}=b \sigma b^{t}$ for some permutation $\sigma$ ), but this factorisation is absent from 
(3.1). Our expression for type 1 is indeed $\mathcal{Z}=b b^{t}$, where $b$ is the manifestly integral matrix for the linear map $\operatorname{Res} \operatorname{Ver}_{\bar{\omega} \bar{\omega} \psi}(\bar{K}) \rightarrow \operatorname{Ver}_{\omega}(G)$ given by (3.3) and expressed in the natural bases $[\bar{k}, \bar{\chi}]$ and $[g, \chi]$. We give an analogous expression in [31] for the modular invariant for general (not necessarily type 1 ) pairs $(H, \psi)$, again using only inductions of characters and decomposition of conjugacy classes of a group into conjugacy classes of a subgroup.

Theorem 1. Let $G$ be any finite group and $\omega \in Z^{3}(G ; \mathbb{T})$ be any 3-cocycle, and define $\widetilde{\omega}$ by $(2.21)$.

(a) Let $H \leq G^{2}$ satisfy $\left.[\widetilde{\omega}]\right|_{H}=[1]$, and choose $\psi \in Z^{2}(H ; \mathbb{T})$. Then $(H, \psi)$ is a type 1 pair for $\mathcal{D}_{\omega}(G)$ iff (up to conjugation in $\left.G^{2}\right) H=\Delta_{K}(1 \times N$ ) for some subgroup $K$ of $G$ and normal subgroup $N$ of $K$, with $\left.[\psi]\right|_{\Delta_{K}}=[1]$ and $\beta_{N \times 1}^{\psi}(1 \times N)=1$. The condition on $\psi$ is equivalent (up to adjustment by coboundary) to requiring that $\psi$ satisfy both $\psi\left(\Delta_{K}, H\right)=1$ and $\psi\left((n, 1),\left(1, n^{\prime}\right)\right)=\psi\left(\left(1, n^{\prime}\right),(n, 1)\right)$ for all $n, n^{\prime} \in N$.

(b) Choose any type 1 pair $(H, \psi)$, with $\psi$ as in the last sentence of $(a)$.

(i) The associated algebra $A$ is described below. The category of right A-modules (the module category) is ${ }_{\Delta_{G}} \mathcal{C}\left(G^{2}\right)_{H}^{\tilde{\omega}, \psi}$, and the category of $A$-A-bimodules (the full system) is ${ }_{H}^{\psi} \mathcal{C}\left(G^{2}\right)_{H}^{\tilde{\omega}, \psi}$. Alpha-induction $\alpha: \mathcal{D}_{\omega}(G) \rightarrow{ }_{\Delta_{G}} \mathcal{C}\left(G^{2}\right)_{H}^{\tilde{\omega}, \psi}$ is

$$
\alpha^{H}([g, \chi])=\sum_{k \in C_{G}(g) \backslash G / K}\left[g^{k}, \operatorname{Ind}_{C_{K}\left(g^{k}\right)}^{C_{H}\left(g^{k}\right)} \chi^{k}\right],
$$

where $C_{H}(g)=\left\{k \in K \mid g^{k} \in N\right\}$. Write (3.2) symbolically as $\sum\left[g^{\prime}, \tilde{\chi}\right]$. The alpha-inductions $\alpha_{ \pm}: \mathcal{D}_{\omega}(G) \rightarrow{ }_{H}^{\psi} \mathcal{C}\left(G^{2}\right)_{H}^{\tilde{\omega}, \psi}$ are $\alpha_{+}[g, \chi]=\sum\left[\left(g^{\prime}, 1\right), \tilde{\chi}_{+}\right]$and $\alpha_{-}[g, \chi]=\sum\left[\left(1, g^{-1}\right), \tilde{\chi}_{-}\right]$, where we write $\tilde{\chi}_{ \pm}\left(h_{+}, h_{-}\right)=\tilde{\chi}\left(h_{ \pm}, h_{ \pm}\right)$.

(ii) Sigma-restriction ${ }_{\Delta_{G}} \mathcal{C}\left(G^{2}\right)_{H}^{\tilde{\omega}, \psi} \rightarrow{ }_{\Delta_{G}} \mathcal{C}\left(G^{2}\right)_{\Delta_{G}}^{\tilde{\omega}}$ sends the bundle $[(g, 1), \chi]$ to $\sum_{n}\left[(n g, 1), \chi^{\prime}\right]$, where the sum is as in the decomposition $\mathrm{cl}_{K}(g) N=\cup_{n} \mathrm{cl}_{K}(n g)$ into orbits $\mathrm{cl}_{K}\left(g^{\prime}\right)$ of $K^{\text {adj }}$ in $G$, and $\chi^{\prime}$ is defined in (4.2). The indecomposable local bundles in ${ }_{{ }_{G}} \mathcal{C}\left(G^{2}\right)_{H}^{\tilde{\omega}, \psi}$ are those bundles $[(k, 1), \chi]$ for which $k \in K$ and $\chi$ satisfies both $|\chi(k, k)|=|\chi(1,1)|$ and (4.3) for all $n \in N$.

(iii) The modular tensor category of local modules for $(H, \psi)$ is braided tensor equivalent to the twisted group double $\mathcal{D}_{\bar{\omega} \bar{\omega} \psi}(\bar{K})$, where $\bar{\omega}$ and $\bar{\omega}^{\psi}$ are 3 -cocycles on $\bar{K}:=K / N$ defined in Proposition 2 (with $G$ there replaced with $K$ ). Sigmarestriction $\operatorname{Ver}_{\bar{\omega} \bar{\omega} \psi}(\bar{K}) \rightarrow \operatorname{Ver}_{\omega}(G)$ is $\iota$ ! $\circ \pi^{*}$ where $\pi^{*}$ resp. $\iota$ ! are the natural $K$-theoretic maps coming from the quotient $\pi$ and inclusion $\iota$ respectively.

$K$-theoretic maps like $\pi^{*}: \operatorname{Ver}_{\bar{\omega} \bar{\omega} \psi}(\bar{K}) \rightarrow \operatorname{Ver}_{\omega}(K)$ and $\iota ! \operatorname{Ver}_{\omega}(K) \rightarrow \operatorname{Ver}_{\omega}(G)$ in Theorem 1(b)(iii) are discussed in section 2.2. Explicitly, choosing coset representatives $t_{\bar{k}} \in \bar{k} \in K / N$, the sigma-restriction $\iota_{!} \circ \pi^{*}$ in Theorem 1(b)(iii) becomes

$$
[\bar{k}, \bar{\chi}] \mapsto \sum_{k}\left[k, \operatorname{Ind}_{C_{K}}^{C_{G}(k)}(k) \bar{\phi} \circ \pi\right]
$$


where the sum over $k$ is as in $\cup_{k} \operatorname{cl}(k)=\pi^{-1}(\operatorname{cl}(\bar{k}))$, and $\widetilde{\phi}(h)=\beta_{(k, k)}\left(h t_{h N}{ }^{-1}, 1\right)^{*}$.

Ostrik's guess [69] for the type 1 module categories missed the $\psi \neq 1$ possibilities. The smallest $G$ for which these arise are the dihedral group $D_{4}$ and the alternating group $A_{4}$ - see section 3.4 for an example. We do not know whether inequivalent module categories $(H, \psi)$ necessarily have distinct modular invariants $\mathcal{Z}$ - this would have seemed very unlikely except that it is true when $\mathcal{Z}$ is a permutation matrix [31].

The MTC of local modules goes by other names in the literature: the ambichiral or neutral system, and more generally the type 1 parents, in subfactor theory; the left and right centres in categorical treatments; the extended system in CFT or VOA. The phrase we use should make sense in all frameworks.

Of the two contributions $\bar{\omega}$ (which depends on $\omega$ ) and $\bar{\omega}^{\psi}$ (the part depending on $\psi$ ) to the 3-cocycle twist in Theorem 1(b)(iii), the more important for us is $\bar{\omega}^{\psi}$. Our proof of reconstruction (Theorem 3) requires delicate control over $\bar{\omega}^{\psi}$ - in fact we get reconstruction (Theorem 3) for arbitrary $\omega$, even if Theorem 1 were to hold only for trivial twist $\omega=1$. A crucial part of our proof connects $\bar{\omega}^{\psi}$ to the thesis of Vaughan Jones [47]. A $Q$-kernel is a homomorphism from a group $Q$ to the outer automorphism group $\operatorname{Out}(M)=\operatorname{Aut}(M) / \operatorname{Int}(M)$ of a factor $M$. Any $Q$-kernel gives rise to a 3-cocycle on $Q$. If one has an action of $K$ by automorphisms on a factor, which is inner when restricted to subgroup $N$, then one has a $K / N$-kernel and hence a 3-cocycle on $K / N$. This is what Jones exploits and computes explicitly. The structure coming along with this framework is necessary for our proof of Theorem 3 .

We find in section 4.3 that the algebra $A$ associated to the type 1 module category $(H, \psi)$ of Theorem 1 consists of a copy $A_{(g n, g)}$ of $\beta_{(n, 1)}^{\psi} \otimes \mathcal{F}\left(C_{G}(n) / C_{K}(n)\right)$ at each point $(g n, g) \in \Delta_{G}\left(1 \times N^{G}\right)$, where $N^{G}$ is the set $\cup_{g \in G} N^{g}$ and $\mathcal{F}\left(G^{\prime} / K^{\prime}\right)$ denotes the algebra of functions on the set $G^{\prime} / K^{\prime}$ of cosets. Multiplication in $\mathcal{F}\left(G^{\prime} / K^{\prime}\right)$ is pointwise, and as a $G^{\prime}$-representation is equivalent to $\operatorname{Ind}_{K^{\prime}}^{G^{\prime}} \mathbf{1}$. The group $\Delta_{G}$ acts on the left and right of $A$ as in (2.16)-(2.18), making $A$ into a bundle in ${ }_{\Delta_{G}} \mathcal{C}\left(G^{2}\right){ }_{\Delta_{G}}^{\tilde{\omega}}$. The multiplication $\mu: A_{(1,1)} \otimes A_{(n, 1)} \rightarrow A_{(n, 1)}$ is that of $\mathcal{F}(G / K)$ restricted to $C_{G}(n)$, and is extended to $\mu: \mathbb{C}_{(g n, g)} \otimes \mathbb{C}_{\left(g^{\prime} n^{\prime}, g^{\prime}\right)} \rightarrow \mathbb{C}_{\left(g g^{\prime} n^{g^{\prime}} n^{\prime}, g g^{\prime}\right)}$ by equivariance

$$
g \mu(v \otimes w) h=\mu(g v \otimes w h) .
$$

It is elementary to specialise Theorem 1 to cyclic groups.

Corollary 1. Let $G \cong \mathbb{Z}_{n}$ and write $\omega=\omega_{q}$ as in (2.13). Then the type 1 module categories correspond to any choice of integers $m, m^{\prime}, a \in \mathbb{Z}_{>0}$, where $m^{\prime}|m| n, a \leq m^{\prime}$, $m^{\prime} \mid q$ and $\left(m^{\prime}\right)^{2} \mid$ am. The MTC of local modules is a twisted double of $\mathbb{Z}_{m / m^{\prime}}$.

Here, if we write $G=\langle g\rangle$, then $K=\left\langle g^{n / m}\right\rangle \cong \mathbb{Z}_{m}$ and $N=\left\langle g^{n / m^{\prime}}\right\rangle \cong \mathbb{Z}_{m^{\prime}}$. The 2-cocycle $\psi$ comes from $a$ and is given explicitly in section 4.6, along with the proof. See also the discussion of [24] in section 3.2. Cyclic $G$ is so easy because all simples in $\mathcal{D}_{\omega}(G)$ are invertible.

The maximal extensions of $\mathcal{D}_{\omega}(G)$ occur when the MTC of local modules is trivial, i.e. is $V_{e c}$. Such type 1 module categories are said to trivialise $\mathcal{D}_{\omega}(G)$, for this reason. They are classified by an analogue of Proposition 1 [69], but again the 
branching rules etc are obscure. In section 4.7 we recover this classification as a corollary of Theorem 1 , and with it the branching rules etc.

Corollary 2. Choose any finite group $G$ and any 3-cocycle $\omega \in Z^{3}(G ; \mathbb{T})$. The type 1 module categories of $\mathcal{D}_{\omega}(G)$ whose $M T C$ of local modules is $\mathrm{Vec}_{\mathbb{C}}$, correspond to any subgroup $K \leq G$ and $\psi^{\prime} \in Z^{2}(K ; \mathbb{T})$ such that $\left.\omega\right|_{K}$ is coboundary. Then

$$
1 \mapsto \sum_{k}\left[k, \beta_{k}^{\psi^{\prime}} \cdot \operatorname{Res}_{C_{G}(k)} \operatorname{Ind}_{K}^{G} \mathbf{1}\right]
$$

is the associated branching rule, where the sum is over conjugacy class representatives of $K$ by $G$, and $\beta^{\psi^{\prime}}$ is defined in (2.7). In the notation of Theorem 1(a), this module category has $H=K \times K$ and cocycle $\psi \in Z^{2}(K \times K ; \mathbb{T})$ given by

$$
\psi\left(\left(k_{1}, k_{2}\right),\left(k_{1}^{\prime}, k_{2}^{\prime}\right)\right)=\psi^{\prime}\left(k_{2}^{\prime-1}, k_{2}^{-1} k_{1}\right) \psi^{\prime}\left(\left(k_{2}^{-1} k_{1}\right)^{k_{2}^{\prime}}, k_{2}^{\prime}\right)^{*} \psi^{\prime}\left(\left(k_{2}^{-1} k_{1}\right)^{k_{2}^{\prime}}, k_{2}^{\prime-1} k_{1}^{\prime}\right) .
$$

In the physics literature, two of these maximal extensions are singled out: the pair $\left(K, \psi^{\prime}\right)=(1,1)$ (the original holomorphic theory) and the pair $\left(K, \psi^{\prime}\right)=(G, 1)$ (what is unfortunately called there the orbifold of the original theory by $G$ ). The physics literature realises that the latter doesn't always exist - in particular so-called levelmatching must be satisfied. This (necessary but not sufficient) condition requires that the resulting modular invariant be invariant under $T$. In hindsight, there is no reason to restrict to those two maximal extensions. Moreover level-matching must be replaced with the $\left.[\omega]\right|_{K}=[1]$ condition, which implies it; in general it is only for cyclic $K$ that level-matching is equivalent to that condition on $\widetilde{\omega}$.

For example, Corollary 2 for $G=\mathbb{Z}_{n}$ reduces to $m^{\prime}=m$ dividing $q$, and $a=0$.

Although Proposition 1 is abstract nonsense (for this reason Ostrik asked his questions), it is significant that the formulae of Theorem 1 yield explicit expressions. Indeed, all alpha-inductions, sigma-restrictions and modular invariant entries are computed by nothing more complicated than induction and restriction of (possibly projective) characters, and decomposition of conjugacy classes of a group into conjugacy classes of a subgroup. All coefficients are manifestly in $\mathbb{Z}_{\geq 0}$. Before turning to the proofs in section 4 , we discuss some applications of Theorem 1 in section 3.2 and collect several concrete examples in section 3.4.

\subsection{Conformal nets and finite group doubles}

This subsection describes some deep consequences of Theorem 1 for conformal nets of factors. See the following subsection for comments concerning VOAs.

Recall that a holomorphic conformal net $\mathcal{A}$ is completely rational with trivial representation theory: $\operatorname{Rep}(\mathcal{A}) \cong \operatorname{Vec}_{\mathbb{C}}$. When $G$ is a finite group of automorphisms, the space $\mathcal{A}^{G}$ of fixed points is automatically a conformal net, called the orbifold of $\mathcal{A}$ by $G$. It is known that $\mathcal{A}^{G}$ is completely rational whenever $\mathcal{A}$ is completely rational [73] (holomorphicity of $\mathcal{A}$ is not needed). When $\mathcal{A}$ is in addition a holomorphic, then the $\operatorname{MTC} \operatorname{Rep}\left(\mathcal{A}^{G}\right)$ is $\mathcal{D}_{\omega}(G)$ for some 3-cocycle $\omega \in Z^{3}(G ; \mathbb{T})$ (Theorem 5.14 in [62]). Corollary 4 below tells us that the converse also holds. 
As explained in the Introduction, a natural question is to describe all conformal (a.k.a. local) extensions of the orbifold $\mathcal{A}^{G}$. Such questions seem very difficult to answer at present, unless one uses categorical methods: the conformal extensions of $\mathcal{A}^{G}$ correspond to the type 1 module categories $[52,43,16]$ in $\operatorname{Rep}\left(\mathcal{A}^{G}\right) \cong \mathcal{D}_{\omega}(G)$. Thus Theorem 1 gives all such extensions.

Corollary 3. Suppose $\mathcal{A}$ is a completely rational conformal net with $\operatorname{Rep}(\mathcal{A})$ tensor equivalent to $\mathcal{D}_{\omega}(G)$. Then the conformal extensions $\mathcal{A}^{e}$ of $\mathcal{A}$ correspond to the type 1 pairs $(H, \psi)$. Their MTC $\operatorname{Rep}\left(\mathcal{A}^{e}\right)$ is a twisted group double identified in Theorem 1(b)(iii). The decomposition (branching rules) of $\mathcal{A}^{e}$ and its representations $\pi \in$ $\operatorname{Rep}\left(\mathcal{A}^{e}\right)$ into a direct sum of $\mathcal{A}$-representations is given in Theorem 1(b)(ii).

Equivalent module categories ${ }_{\Delta_{G}} \mathcal{C}\left(G^{2}\right)_{H}^{\tilde{\omega}, \psi}$ (i.e. conjugate pairs $\left.(H, \psi)\right)$ correspond to extensions of $\mathcal{A}$ equivalent as $\mathcal{A}$-representations (and also as conformal nets), but inequivalent pairs $(H, \psi)$ may correspond to equivalent conformal nets - see e.g. the $A_{2} \oplus E_{6}$ lattice example given in section 3.4.

Some of these extensions are clear. The quantum Galois correspondence (given in $[40,22]$ for VOAs but it applies equally for conformal nets) says that all conformal nets $\mathcal{B}$ between $\mathcal{A}^{G}$ and $\mathcal{A}$ are in natural bijection with subgroups $K$ of $G$, namely $K \leftrightarrow \mathcal{A}^{K}$. These correspond to the type 1 module categories with $(H, \psi)=\left(\Delta_{K}, 1\right)$. But Theorem 1 tells us that there are several other conformal extensions $\left(\mathcal{A}^{G}\right)^{e}$ of $\mathcal{A}^{G}$ which aren't subnets of $\mathcal{A}$. Much more challenging is to obtain these from traditional (i.e. noncategory-theoretic) conformal net or VOA constructions. The plethora of extensions contained in Theorem 1 provides a graphic example of what seems to be a serious general challenge for VOAs and conformal nets: to find new construction methods. See e.g. [28] for further conformal net and VOA candidates (realised by braided subfactors and MTC) that have not yet been constructed.

The simplest class of these less obvious extensions is as follows. Let $\mathcal{A}$ be holomorphic, $G$ a finite group of automorphisms and $\operatorname{Rep}\left(\mathcal{A}^{G}\right) \cong \mathcal{D}_{\omega}(G)$, as before. Assume for simplicity here that $\omega=1$ - nontrivial $\omega$ is handled by Theorem 1 , and excludes some of the following extensions. There exists a completely rational conformal net $\mathcal{A}_{N}^{G}$ containing $\mathcal{A}^{G}$, for each normal subgroup $N \triangleleft G$. In this case the category of $\mathcal{A}_{N}^{G}$-modules is the double $\mathcal{D}_{1}(G / N)$. Any simple $\mathcal{A}_{N}^{G}$-module decomposes as a $\mathcal{A}^{G}$ module into the direct sum (4.13). More generally, one can twist these $\mathcal{A}_{N}^{G}$ by Jones' characteristic invariant (the $\psi$ in Theorem 1(a)), and this can introduce a nontrivial 3-cocycle $\bar{\omega}^{\psi}$ even when one wasn't there before. The general extension of $\mathcal{A}^{G}$ is a combination of the $\mathcal{A}^{H}$ and these $\mathcal{A}_{N, \psi}^{G}$.

Corollary 2 gives all holomorphic (i.e. maximal) extensions of $\mathcal{A}^{G}$. There may only be one of these, namely the original $\mathcal{A}$ itself, corresponding to $K=1$. The converse, which was previously known, is also true:

Corollary 4. Suppose $\mathcal{A}$ is a completely rational conformal net with $\operatorname{Rep}(\mathcal{A})$ tensor equivalent to $\mathcal{D}_{\omega}(G)$. Then $\mathcal{A}$ is an orbifold by $G$ of a holomorphic net.

To see this, choose the holomorphic extension $\mathcal{A}^{\text {hol }}$ of $\mathcal{A}$ associated to $K=1$ and $\psi=1$ in the notation of Corollary 2. Then as an $\mathcal{A}$-representation, $\mathcal{A}^{\text {hol }}=$ 
$\oplus_{\pi \in \operatorname{Irr}(G)} \pi \otimes[1, \pi]$ using obvious notation. But each summand $\pi \otimes[1, \pi]$ manifestly carries a $G \times \mathcal{A}$-action, and it is evident that $\left(\mathcal{A}^{\text {hol }}\right)^{G}=[1, \mathbf{1}]=\mathcal{A}$. QED to Corollary 4

The best studied examples of orbifolds are permutation orbifolds. Let $\mathcal{A}$ be any conformal net. Let $G$ be any subgroup of the symmetric group $S_{k}$. Then $\left(\mathcal{A}^{\otimes k}\right)^{G}$ is called a permutation orbifold - here $G$ acts by $v_{1} \otimes \cdots \otimes v_{k} \mapsto v_{\pi 1} \otimes \cdots \otimes v_{\pi k}$ in the $k$-fold tensor product.

We are interested in the special case where $\mathcal{A}$ is holomorphic. In this case the central charge $c$ necessarily lies in $8 \mathbb{Z}_{\geq 0}$. The categories $\operatorname{Rep}\left(\left(\mathcal{A}^{\otimes k}\right)^{G}\right)$ is $\mathcal{D}_{\omega}(G)$ up to some twist $\omega \in Z^{3}(G ; \mathbb{T})$. In Theorem 2 we identify this twist $\omega$ for any such permutation orbifold.

Theorem 2. Let $\mathcal{A}$ be a holomorphic conformal net of central charge $c$, and let $G$ be a subgroup of some symmetric group $S_{k}$. Let $\left(\mathcal{A}^{\otimes k}\right)^{G}$ be the corresponding permutation orbifold, and let $\mathcal{D}_{\omega}(G)$ be its category of modules. Then $[\omega]$ is the restriction to $G$ of $\left[\omega_{c(\bmod 3)}^{(3)}\right] \in H^{3}\left(S_{k} ; \mathbb{T}\right)$, where the 3-torsion cocycle $\omega_{q}^{(3)}$ is defined in section 4.8 . In particular, if 24 divides c or 3 does not divide the order $|G|$ of $G$, then the twist $\omega$ is trivial.

We thank Marcel Bischoff for correspondence on the possibility that $[\omega] \neq[1]$. The expectation had been that $[\omega]=1$ always $[63,18]$. Both Bischoff and JohnsonFreyd (the latter in [45]) also showed the 3-cocycle could be nontrivial in general. Example 2.1.1 in [45] announces the general result, corresponding to our Theorem 2.

The nontrivial twist can be detected by the character vectors. For example, fix some holomorphic theory $\mathcal{A}$ at central charge $c \in 8 \mathbb{Z}$ and some $n \geq 1$, with graded dimension $\chi_{\mathcal{A}}(\tau)$, and write $\pi=(12 \cdots n) \in S_{n}$. Consider the $\mathbb{Z}_{n}$-permutation orbifold $\mathcal{A}_{\pi}=\left(\mathcal{A}^{\otimes n}\right)^{\langle\pi\rangle}$. Then $\mathcal{A}_{\pi}$ has graded dimension

$$
\chi_{\mathcal{A}_{\pi}}(\tau)=\sum_{d \mid n} \frac{\phi(d)}{n} \chi_{\mathcal{A}}(d \tau)^{n / d}
$$

where $\phi(d)$ is the Euler totient and the sum is over all divisors of $n$ (see equation (4.15) of [10] for an analogous expression for the character of any $\mathcal{A}_{\pi}$-module). Now, $\chi_{\mathcal{A}}(-1 / \tau)$ must be a linear combination with nonzero coefficients of the characters of $\mathcal{A}$-representations, and so from it we can read off all values appearing in the modular $T$ matrix for $\mathcal{A}$. We see that

$$
\chi_{\mathcal{A}_{\pi}}(-1 / \tau)=\sum_{d \mid n} \frac{\phi(d)}{n} \chi_{\mathcal{A}}(\tau / d)^{n / d} \in \sum_{d \mid n} q^{-n c / 24 d^{2}} \mathbb{C}\left[\left[q^{1 / d}\right]\right] .
$$

The central charge of $\mathcal{A}_{\pi}$ is $n c$, and we see that $e^{2 \pi i n c / 24} T$ for $\mathcal{A}_{\pi}$ has order $n$, unless both $3 \mid n$ and 3 doesn't divide $c$, in which case the order is $3 n$. But $T$ should match $T^{\omega_{q}}$ in (2.14) for some $0 \leq q<n$, so $q=0$ unless both $3 \mid n$ and $3 \not c$, in which case $q=c n / 3$.

Perhaps the biggest question on the interface of conformal nets/VOAs and categories is reconstruction: is every MTC realised by a completely rational VOA or 
conformal net? The first nontrivial place to look, presumably, is to the finite group categories $\mathcal{D}_{\omega}(G)$, for arbitrary $G$ and $\omega$. These would correspond to orbifolds by $G$ of a holomorphic VOA or conformal net.

It had been expected for some time that permutation orbifolds would realise any $\mathcal{D}_{1}(G)$, and this is now proven (Theorem 2). But until now, it has been far from clear that $\mathcal{D}_{\omega}(G)$ for all 3-cocycles $\omega$ and all finite groups $G$, can likewise be realised as a representation category. Indeed, the 3-cocycles appearing in all flavours of Moonshine all seem to have order dividing 24 .

Theorem 3. For any finite group $G$ and any 3-cocycle $\omega \in Z^{3}(G ; \mathbb{T})$, there exists a completely rational conformal net $\mathcal{A}$ whose representation category $\operatorname{Rep}(\mathcal{A})$ is $\mathcal{D}_{\omega}(G)$.

We prove Theorems 2 and 3 in section 4 .

\subsection{VOAs and finite group doubles}

This subsection does for vertex operator algebras (VOAs) what the previous subsection does for conformal nets. In general, less is known about the structure theory of VOAs than of conformal nets.

Recall that a holomorphic VOA $\mathcal{V}$ is a completely rational VOA with trivial representation theory: $\operatorname{Mod}(\mathcal{V}) \cong \operatorname{Vec}_{\mathbb{C}}$. When $G$ is a finite group of automorphisms, the space $\mathcal{V}^{G}$ of fixed points is automatically a VOA, called the orbifold of $\mathcal{V}$ by $G$.

Conjecture 1. Let $\mathcal{V}$ be a holomorphic VOA and $G$ be a finite group of automorphisms of $G$. Then the orbifold $\mathcal{V}^{G}$ is also completely rational.

In fact it is generally believed that $\mathcal{V}^{G}$ is completely rational whenever $\mathcal{V}$ is completely rational (and not necessarily holomorphic) and $G$ is finite. This has been proved $[60,12]$ when $G$ is solvable.

Conjecture 2. Let $\mathcal{V}$ be a holomorphic VOA and $G$ a finite group of automorphisms. Assume $\mathcal{V}^{G}$ is completely rational (Conjecture 1). Then the $M T C \operatorname{Rep}\left(\mathcal{V}^{G}\right)$ is $\mathcal{D}_{\omega}(G)$ for some 3-cocycle $\omega \in Z^{3}(G ; \mathbb{T})$.

This also is generally believed. Proposition 5.6 of [23] establishes this for all cyclic $G$, using intrinsically VOA methods. The main theorem of [52] shows this for any $G$ but trivial twist $\omega$ (i.e. all 2-cocycles arising are 1) - his proof is categorical, so works equally for conformal nets and (assuming Conjecture 1) VOAs.

If Conjectures 1 and 2 hold, then everything in section 3.2 has an analogue for VOAs.

First of all, what is the VOA analogue of Corollary 3? The same categorical methods apply, however [43] requires that we impose the additional property:

(*) every simple $\mathcal{V}$-module $M \neq \mathcal{V}$ must have conformal weight $>0$.

This is not an issue in the conformal net picture, because of unitarity. Modulo this condition, the analogue of Corollary 3 holds for VOAs. In fact $(*)$ is stronger than necessary: what is required is that any simple $\mathcal{V}$-module $M \neq \mathcal{V}$ appearing in 
any commutative algebra $A$ defining a conformal extension of $\mathcal{V}$ (i.e. any simple $M$ appearing in the restriction of an extension $\mathcal{V}^{e}$ to $\mathcal{V}$ ), have conformal weight $>0$.

Subject to this condition $(*)$, Corollary 2 gives all holomorphic (i.e. maximal) extensions of any completely rational $\operatorname{VOA} \mathcal{V}$ with $\operatorname{Mod}(\mathcal{V})$ tensor equivalent to $\mathcal{D}_{\omega}(G)$. The case of cyclic $G$ was studied recently in [24]. This paper plays an important role both in the classification of holomorphic VOAs with central charge 24 (they construct 5 new ones), and in the proof of Generalised Moonshine. Their Theorem 5.16 describes all type 1 module categories - because all simple modules in $\mathcal{D}_{\omega}(G)$ are invertible when $G$ is cyclic, here it is straightforward to get the complete story. Holomorphic extensions of cyclic orbifolds, which share the additional key property of the Monstrous Moonshine VOA that there are few states with small conformal weights, have recently been studied in [37].

Certainly it is expected that Theorem 2, which describes the twist of permutation orbifolds of holomorphic theories, is expected to hold for VOAs. It will, if both Conjectures 1 and 2 hold. At present we don't have a proof of the VOA analogue of Theorem 2, even in the case where $G$ is solvable.

We are able, however, to prove the following VOA analogue of Theorem 3:

Theorem 4. When $G$ is solvable, then for any $\omega \in Z^{3}(G ; \mathbb{T})$ there exists a completely rational $\operatorname{VOA} \mathcal{V}$ with $\operatorname{Mod}(\mathcal{V}) \cong \mathcal{D}_{\omega}(G)$.

Our proof of Theorem 4, which is given in section 4, assumes neither Conjectures 1 nor 2. The reason solvable groups appear, is because it is known that VOA orbifolds by solvable groups preserves the property of completely rational. If it is proven that the orbifold of any completely rational VOA by e.g. an alternating group $A_{n}$ is also completely rational, then the proof of Theorem 4 extends to sny finite group with composition factors $\mathbb{Z}_{p}$ and/or $A_{n}$.

\subsection{Examples}

Consider any type 1 module category, i.e. any pair $(H, \psi)$ in Theorem 1 (a). The simple objects in the extended theory, thanks to Theorem $1(\mathrm{~b})$ (iii), are $[\bar{k}, \bar{\chi}]$, where $\bar{k} \in \bar{K}=K / N$, and $\bar{\chi}$ is a projective character of $C_{\bar{K}}(\bar{k})$ with 2-cocycle (2.5). Sigmarestriction, and with it the modular invariant, are determined from (3.3).

Let's turn next to some concrete examples. The trivial module category for $\mathcal{D}_{\omega}(G)$, corresponding to the unextended conformal net or VOA, and the diagonal modular invariant $\mathcal{Z}=I$, is associated to the choice $H=\Delta_{G}$ and $\psi=1$.

Another example which works for any $\mathcal{D}_{\omega}(G)$ is $H=1 \times 1$ and $\psi=1$. In this case, there is only one local module (so the extended theory is holomorphic), with sigmarestriction $[1, \mathbf{1}] \mapsto \sum_{\pi} \operatorname{dim} \pi[1, \pi] \in \operatorname{Ver}_{\omega}(G)$, where the sum is over all (linear) characters $\pi \in \operatorname{Irr}(G)$. Note that the 2-cocycle multiplier for these $\pi$ is trivial even when $\omega$ is nontrivial. This example appeared in equation (1.19) of [28].

Another holomorphic example (which works though only for $\omega=1$ ) is $H=G \times G$. Take $\psi=1$ for simplicity. Then sigma-restriction sends the unit to $\sum_{g}[g, \mathbf{1}]$ where 
the sum is over conjugacy class representatives $g$. This example appeared in equation (1.20) of [28].

For comparison, a simple example which is not type 1 is $H=G \times 1$ and $\psi=1$. Then we have two type 1 parents (a.k.a. left and right centres): one is the $H=1$ example whilst the other is $H=G \times G$. Equation (2.2) becomes $\mathcal{Z}=b_{1} b_{G \times G}^{t}$ where $b_{1}$ resp. $b_{G \times G}$ are the matrices capturing the restrictions of the previous two paragraphs.

For a type 1 example with a nontrivial $\psi$, consider $H=A_{4} \times A_{4}$, where $G=A_{4}$ is the alternating group of order 12 and $\omega=1$. Now, $H^{2}\left(A_{4} ; \mathbb{T}\right) \cong \mathbb{Z}_{2}$, so let $\psi^{\prime \prime}$ be the nontrivial one. $A_{4}$ has four conjugacy classes: those of (1), (12)(34), (123) and (132), with stabilisers $A_{4}, \mathbb{Z}_{2} \times \mathbb{Z}_{2}, \mathbb{Z}_{3}, \mathbb{Z}_{3}$ respectively. $\mathcal{D}_{1}\left(A_{4}\right)$ has 14 sectors but most aren't relevant here. The map $\beta^{\psi^{\prime \prime}}$ is trivial on the stabilisers $\mathbb{Z}_{3}$, but restricts to the unique nontrivial alternating bicharacter on $\mathbb{Z}_{2}^{2}$ (this follows from Theorem 2 in [44] or Lemma 1(b) in [30]). We find that the sigma-restrictions of these two module categories are slightly different:

$$
\begin{aligned}
& \psi^{\prime}=1:[1, \mathbf{1}] \mapsto[1, \mathbf{1}]+[(12)(34),++]+[(123), \mathbf{1}]+[(132), \mathbf{1}], \\
& \psi^{\prime}=\psi^{\prime \prime}:[1, \mathbf{1}] \mapsto[1, \mathbf{1}]+\left[(12)(34),{ }_{+-}\right]+[(123), \mathbf{1}]+[(132), \mathbf{1}] \text {, }
\end{aligned}
$$

where we write $s_{1} s_{2}$ for the one-dimensional representation of $\mathbb{Z}_{2}^{2}$ sending $(1,0) \mapsto s_{1}$ and $(0,1) \mapsto s_{2}$, and $\psi^{\prime}$ is as in Corollary 2 .

Consider next the lattice conformal nets $\mathcal{A}\left(A_{2} \oplus E_{6}\right), \mathcal{A}\left(A_{8}\right)$ and $\mathcal{A}(L)$ where $L=\operatorname{span}\left\{D_{7} \oplus \sqrt{36} \mathbb{Z},\left(\frac{1}{2}, \frac{1}{2}, \frac{1}{2}, \frac{1}{2}, \frac{1}{2} ; \frac{9}{\sqrt{36}}\right)\right\}$. These are all $\mathbb{Z}_{3}$ orbifolds of the holomorphic conformal net $\mathcal{A}\left(E_{8}\right)$, and their categories of modules correspond to the three inequivalent twisted doubles of $\mathbb{Z}_{3}$, namely $\mathcal{D}_{\omega_{0}}\left(\mathbb{Z}_{3}\right), \mathcal{D}_{\omega_{1}}\left(\mathbb{Z}_{3}\right)$ and $\mathcal{D}_{\omega_{2}}\left(\mathbb{Z}_{3}\right)$ respectively using the notation of (2.13). According to Theorem 1, there are precisely two nontrivial extensions of $\mathcal{A}\left(A_{2} \oplus E_{6}\right)$ (i.e. type 1 module categories of $\left.\mathcal{D}_{\omega_{0}}\left(\mathbb{Z}_{3}\right)\right)$, namely $[0,0] \oplus[1,1] \oplus[2,2]=\mathcal{A}\left(E_{8}\right)(H=1, \psi=1)$ and $[0,0] \oplus[1,2] \oplus[2,1] \cong \mathcal{A}\left(E_{8}\right)$ $\left(H=\mathbb{Z}_{3} \times \mathbb{Z}_{3}, \psi=1\right)$. Here we write [0], [1], [2] for the simple modules of both $\mathcal{A}\left(A_{2}\right)$ and $\mathcal{A}\left(E_{6}\right)$, and denote by $\left[k, k^{\prime}\right]=[k] \otimes\left[k^{\prime}\right]$ the simple modules of $\mathcal{A}\left(A_{2} \oplus E_{6}\right)$. According to Theorem 1 , there is precisely one nontrivial extension of $\mathcal{A}\left(A_{8}\right)$ (i.e. type 1 module category of $\left.\mathcal{D}_{\omega_{1}}\left(\mathbb{Z}_{3}\right)\right)$, namely $[0] \oplus[3] \oplus[6]=\mathcal{A}\left(E_{8}\right)(H=1, \psi=1)$. Here $[0],[1], \ldots,[8]$ denote the simple modules of $\mathcal{A}\left(A_{8}\right)$, enumerated so that fusion is addition mod 9. Similarly, there is only 1 for $\mathcal{A}(L)$, namely $H=1, \psi=1$, with $\oplus_{j=0}^{2}\left[0, \frac{9 j}{\sqrt{36}}\right] \cong \mathcal{A}\left(E_{8}\right)$. This illustrates a general principle: nontrivial 3-cocycles obstruct extensions.

Consider now the symmetric group $G=S_{3}$. Then $H^{3}\left(S_{3} ; \mathbb{T}\right) \cong \mathbb{Z}_{6}$, with explicit 3cocycles $\omega_{k}$ given in e.g. equation (6.20) of [15]. For readability, in Table 1 we write $\mathcal{D}_{k}$ for $\mathcal{D}_{\omega_{k}}$. That table collects together the type 1 module categories for each of these 6 twisted doubles. In all cases, $\psi$ must be trivial. The column 'MTC' is the MTC of the local modules, as always a twisted group double $\mathcal{D}_{\omega_{q}}(F)$. Conveniently, $H^{2}(K ; \mathbb{T})=0$ for all subgroups $K \leq S_{3}$, which means all 2-cocycles $c_{g}^{\omega}$ are coboundary, so all characters are (projectively) equivalent to linear ones, and we can give all sectors uniform names regardless of $\omega_{k}$. In particular, enumerate the 8 sectors of $\mathcal{D}_{\omega_{k}}\left(S_{3}\right)$ as $\chi_{0}=[1,1], \chi_{1}=[1, s g n], \chi_{2}=[1, \tau], \chi_{3+k}=\left[(123), \xi^{k}\right], \chi_{6+l}=\left[(12),(-1)^{l}\right]$, as in 
[32]; label the $n^{2}$ sectors of $\mathcal{D}_{\omega_{q}}\left(\mathbb{Z}_{n}\right)$ as $\left[i, \phi_{j}\right]$ where $0 \leq i, j<n$ and $\phi_{j}(1)=e^{2 \pi i j / n}$. In the column 'branching rules', we list the sigma-restrictions of each sector of $\mathcal{D}_{\omega_{q}}(F)$ in order; for $F \cong \mathbb{Z}_{n}$ we order the sectors $\left[i, \phi_{j}\right]$ lexicographically. We use the fact that, for each prime $p$, the restriction of the $p$-primary part of $H^{3}(G ; \mathbb{T})$ to $H^{3}(P ; \mathbb{T})$ is injective, where $P$ is a $p$-Sylow subgroup of $G$. The module categories for $\omega=1$ were first given in [32].

Table 1. Type 1 module categories for $\mathcal{D}_{\omega_{k}}\left(S_{3}\right)$

\begin{tabular}{|c|c|c|c|}
\hline$\omega_{k}$ & $H$ & branching rules & MTC \\
\hline$\omega_{0}$ & 1 & $\chi_{0}+\chi_{1}+2 \chi_{2}$ & Vec \\
\hline$\omega_{0}$ & $\Delta\left(\mathbb{Z}_{2}\right)$ & $\chi_{0}+\chi_{2}, \chi_{1}+\chi_{2}, \chi_{6}, \chi_{7}$ & $\mathcal{D}_{0}\left(\mathbb{Z}_{2}\right)$ \\
\hline$\omega_{0}$ & $\mathbb{Z}_{2} \times \mathbb{Z}_{2}$ & $\chi_{0}+\chi_{2}+\chi_{6}$ & Vec \\
\hline$\omega_{0}$ & $\Delta\left(\mathbb{Z}_{3}\right)$ & $\chi_{0}+\chi_{1}, \chi_{2}, \chi_{2}, \chi_{3}, \chi_{4}, \chi_{5}, \chi_{3}, \chi_{4}, \chi_{5}$ & $\mathcal{D}_{0}\left(\mathbb{Z}_{3}\right)$ \\
\hline$\omega_{0}$ & $\mathbb{Z}_{3} \times \mathbb{Z}_{3}$ & $\chi_{0}+\chi_{1}+2 \chi_{3}$ & Vec \\
\hline$\omega_{0}$ & $\Delta\left(S_{3}\right)$ & $\chi_{i}$ & $\mathcal{D}_{0}\left(S_{3}\right)$ \\
\hline$\omega_{0}$ & $\Delta\left(S_{3}\right)\left(\mathbb{Z}_{3} \times 1\right)$ & $\chi_{0}+\chi_{3}, \chi_{1}+\chi_{3}, \chi_{6}, \chi_{7}$ & $\mathcal{D}_{0}\left(\mathbb{Z}_{2}\right)$ \\
\hline$\omega_{0}$ & $S_{3} \times S_{3}$ & $\chi_{0}+\chi_{3}+\chi_{6}$ & Vec \\
\hline$\omega_{3}$ & 1 & $\chi_{0}+\chi_{1}+2 \chi_{2}$ & Vec \\
\hline$\omega_{3}$ & $\Delta\left(\mathbb{Z}_{2}\right)$ & $\chi_{0}+\chi_{2}, \chi_{1}+\chi_{2}, \chi_{6}, \chi_{7}$ & $\mathcal{D}_{1}\left(\mathbb{Z}_{2}\right)$ \\
\hline$\omega_{3}$ & $\Delta\left(\mathbb{Z}_{3}\right)$ & $\chi_{0}+\chi_{1}, \chi_{2}, \chi_{2}, \chi_{3}, \chi_{4}, \chi_{5}, \chi_{3}, \chi_{4}, \chi_{5}$ & $\mathcal{D}_{0}\left(\mathbb{Z}_{3}\right)$ \\
\hline$\omega_{3}$ & $\mathbb{Z}_{3} \times \mathbb{Z}_{3}$ & $\chi_{0}+\chi_{1}+2 \chi_{3}$ & Vec \\
\hline$\omega_{3}$ & $\Delta\left(S_{3}\right)$ & $\chi_{i}$ & $\mathcal{D}_{3}\left(S_{3}\right)$ \\
\hline$\omega_{3}$ & $\Delta\left(S_{3}\right)\left(\mathbb{Z}_{3} \times 1\right)$ & $\chi_{0}+\chi_{3}, \chi_{1}+\chi_{3}, \chi_{6}, \chi_{7}$ & $\mathcal{D}_{1}\left(\mathbb{Z}_{2}\right)$ \\
\hline$\overline{\omega_{ \pm 2}}$ & 1 & $\chi_{0}+\chi_{1}+2 \chi_{2}$ & Vec \\
\hline$\overline{\omega_{ \pm 2}}$ & $\Delta\left(\mathbb{Z}_{2}\right)$ & $\chi_{0}+\chi_{2}, \chi_{1}+\chi_{2}, \chi_{6}, \chi_{7}$ & $\mathcal{D}_{0}\left(\mathbb{Z}_{2}\right)$ \\
\hline$\omega_{ \pm 2}$ & $\mathbb{Z}_{2} \times \mathbb{Z}_{2}$ & $\chi_{0}+\chi_{2}+\chi_{6}$ & Vec \\
\hline$\overline{\omega_{ \pm 2}}$ & $\Delta\left(\mathbb{Z}_{3}\right)$ & $\chi_{0}+\chi_{1}, \chi_{2}, \chi_{2}, \chi_{3}, \chi_{4}, \chi_{5}, \chi_{3}, \chi_{4}, \chi_{5}$ & $\mathcal{D}_{ \pm 1}\left(\mathbb{Z}_{3}\right)$ \\
\hline$\overline{\omega_{ \pm 2}}$ & $\Delta\left(S_{3}\right)$ & $\chi_{i}$ & $\mathcal{D}_{ \pm 2}\left(S_{3}\right)$ \\
\hline$\overline{\omega_{ \pm 1}}$ & 1 & $\chi_{0}+\chi_{1}+2 \chi_{2}$ & Vec \\
\hline$\overline{\omega_{ \pm 1}}$ & $\Delta\left(\mathbb{Z}_{2}\right)$ & $\chi_{0}+\chi_{2}, \chi_{1}+\chi_{2}, \chi_{6}, \chi_{7}$ & $\mathcal{D}_{1}\left(\mathbb{Z}_{2}\right)$ \\
\hline$\overline{\omega_{ \pm 1}}$ & $\Delta\left(\mathbb{Z}_{3}\right)$ & $\chi_{0}+\chi_{1}, \chi_{2}, \chi_{2}, \chi_{3}, \chi_{4}, \chi_{5}, \chi_{3}, \chi_{4}, \chi_{5}$ & $\mathcal{D}_{ \pm 1}\left(\mathbb{Z}_{3}\right)$ \\
\hline$\overline{\omega_{ \pm 1}}$ & $\Delta\left(S_{3}\right)$ & $\chi_{i}$ & $\mathcal{D}_{ \pm 1}\left(S_{3}\right)$ \\
\hline
\end{tabular}

\section{Proofs}

By a transversal $t_{\bar{k}}$ for $K / N$ we mean a choice of coset representatives $t_{\bar{k}} \in \bar{k} \subset K$. Write $\beta$ for the quantity $\beta^{\psi}$ defined in $(2.7), c_{g}$ for the cocycle $c_{g}^{\omega}$ of $(2.5)$, and define $\widetilde{\omega}$ by $(2.21)$.

There are two different sorts of extensions in Theorem 1, what we call type $1 \Delta$ (arising in the quantum Galois correspondence) and type $1 N$. The general extension is a combination of these. Type $1 \Delta$ has $H=\Delta_{K}$ and $\psi=1$, for any subgroup $K \leq G$ (no constraint comes from $\omega$ ). Type $1 N$ has $H=\Delta_{G}(1 \times N)$ where $N \unlhd G$ is normal, 
with $\psi(G, H)=1, \beta_{N \times 1}^{\psi}(1 \times N)=1$ and $[\widetilde{\omega}]_{H}=[1]$.

\subsection{Type $1 \Delta$}

We will study the general case through the type $1 N$ module category $\left(\Delta_{K}(1 \times N), \psi\right)$ of $\mathcal{D}_{\omega}(K)$, transferred to $\mathcal{D}_{\omega}(G)$ through the type $1 \Delta$ module category $\left(\Delta_{K}, 1\right)$ of $\mathcal{D}_{\omega}(G)$. For this reason, we need to first understand the type $1 \Delta$ case.

Given any pair $(H, \psi)$, Proposition 1 associates the $\mathcal{D}_{\omega}(G)$ module category ${ }_{\Delta_{G}} \mathcal{C}\left(G^{2}\right)_{H}^{\tilde{\omega}, \psi}$ and full category ${ }_{H}^{\psi} \mathcal{C}\left(G^{2}\right)_{H}^{\tilde{\omega}, \psi}$. Any module category is associated an associative algebra $A=\theta$ in $\mathcal{D}_{\omega}(G)$; the module category is type 1 iff $A$ is commutative in the braided sense $\mu_{A} \circ c_{A, A}=\mu_{A}$. The general relation between (bi)modules and bundles that we need is worked out in Lemma 3.1 of [32].

Consider first type $1 \Delta$, so $H=\Delta_{K}$. By $\mathcal{F}(G / K)$ we mean the algebra of functions on the set $G / K$ of left cosets. The multiplication in this algebra is pointwise, $\left(f_{1} f_{2}\right)([g])=f_{1}([g]) f_{2}([g])$ (hence is commutative). $\mathcal{F}(G / K)$ carries a (true) representation of $G$, namely $\operatorname{Ind}_{K}^{G} \mathbf{1}$, obtained through the $G$-action on $G / K$. The desired algebra $A_{\Delta}=\theta_{\Delta}$, which must be a bundle in ${ }_{\Delta_{G}} \mathcal{C}\left(G^{2}\right)_{\Delta_{G}}^{\tilde{\tilde{\omega}}}$, is a copy of $\mathcal{F}(G / K)$ on each point of $\Delta_{G}$, with $\Delta_{G}^{L \times R}$ action given by (2.16)-(2.18) and algebra multiplication $\mu_{\Delta}$ from equivariance (3.4), both applied to the fibre $\mathcal{F}(G / K)$ at $(1,1) \in G^{2}$. As a sector in $\mathcal{D}_{\omega}(G)$, it is $\left[1, \operatorname{Ind}_{K}^{G} \mathbf{1}\right]$. The indecomposable right $A_{\Delta}$-modules in ${ }_{\Delta_{G}} \mathcal{C}\left(G^{2}\right){ }_{\Delta_{G}}^{\tilde{\omega}}$ are precisely those bundles in ${ }_{\Delta_{G}} \mathcal{C}\left(G^{2}\right)_{\Delta_{G}}^{\tilde{\omega}_{G}}$ of the form $\left[(g, 1), \operatorname{Ind}_{C_{K}(g)}^{C_{G}(g)} \bar{\chi}\right]$ where $\bar{\chi} \in \operatorname{Irr}_{c_{g}}\left(C_{K}(g)\right)$; this $A_{\Delta}$-module corresponds to the indecomposable bundle $[(g, 1), \bar{\chi}] \in{ }_{\Delta_{G}} \mathcal{C}\left(G^{2}\right)_{\Delta_{K}}^{\tilde{\omega}}$. That correspondence defines the equivalence between the category of right $A_{\Delta}$-modules in ${ }_{\Delta_{G}} \mathcal{C}\left(G^{2}\right)_{\Delta_{G}}^{\tilde{\omega}}$ and the category ${ }_{\Delta_{G}} \mathcal{C}\left(G^{2}\right)_{\Delta_{K}}^{\tilde{\omega}}$. $A_{\Delta}$ is indeed commutative in the braided sense because the fibre $\mathcal{F}(G / K)$ above the orbit representative $(1,1)$ is a commutative algebra in the classical sense. Thus $(H, \psi)=\left(\Delta_{K}, 1\right)$ is type 1 , as desired.

The above bijection also gives sigma-restriction, which is the functor from $A$ modules to objects in $\mathcal{D}_{\omega}(G)$, forgetting the $A$-module structure. In full generality, it sends $\left[\left(g, g^{\prime}\right), \bar{\chi}\right] \in{ }_{\Delta_{G}} \mathcal{C}\left(G^{2}\right)_{\Delta_{K}}^{\tilde{\omega}}$, where $\bar{\chi}$ is a (projective) representation of the stabiliser ${ }^{\left(g, g^{\prime}\right)} \Delta_{K} \cap \Delta_{G} \cong C_{K}\left(g^{\prime-1} g\right)$, to the bundle $\left[\left(g, g^{\prime}\right), \operatorname{Ind}_{\left(g, g^{\prime}\right) \Delta_{K} \cap \Delta_{G}}^{\left(g, g^{\prime}\right)} \bar{\chi}\right] \in{ }_{\Delta_{G}} \mathcal{C}\left(G^{2}\right)_{\Delta_{G}}^{\tilde{\omega}}$ (note that ${ }^{\left(g, g^{\prime}\right)} \Delta_{G} \cap \Delta_{G} \cong C_{G}\left(g^{\prime-1} g\right)$ ). This coincides with the sigma-restriction given in [29] for the module category $\left(\Delta_{K}, 1\right)$.

The local $A$-modules in ${ }_{\Delta_{G}} \mathcal{C}\left(G^{2}\right)_{\Delta_{G}}^{\tilde{\omega}}$ are precisely those whose sigma-restriction has a well defined twist, or $T$-eigenvalue (see Theorem 3.2 of [53]). It is clear that any $A_{\Delta^{-}}$ module of the form $\left[(k, 1), \operatorname{Ind}_{C_{K}(k)}^{C_{G}(k)} \bar{\chi}\right]$ for $k \in K$ is local: $k \in Z\left(C_{G}(k)\right) \cap Z\left(C_{K}(k)\right)$ so any subrepresentation of $\operatorname{Ind}_{C_{K}(k)}^{C_{G}(k)} \bar{\chi}$ has the same value of twist, namely $\bar{\chi}(k) / \bar{\chi}(1)$. In terms of the full system ${ }_{\Delta_{K}} \mathcal{C}\left(G^{2}\right)_{\Delta_{K}}^{\tilde{\omega}}$, this corresponds to the bundle $[(k, 1), \bar{\chi}]$. This class of local $A_{\Delta}$-modules are precisely those which are also bundles over the subgroupoid ${ }_{\Delta_{K}} \mathcal{C}\left(K^{2}\right)_{\Delta_{K}}^{\tilde{\omega}_{K}}$; these form the MTC $\mathcal{D}_{\omega}(K)$. The easiest way to see that these exhaust all local $A_{\Delta}$-bundles, is to compute dimensions: Theorem 4.5 of [53] tells us the global dimension of the MTC of local $A_{\Delta}$-bundles, which by definition 
is the sum of squares of the quantum-dimensions of all of its sectors, equals the global dimension of $\mathcal{D}_{\omega}(G)$ (which is $|G|^{2}$ ) divided by the square of the quantumdimension of $A_{\Delta}$ in $\mathcal{D}_{\omega}(G)$, which is $|G / K|$ (the quantum-dimension of a bundle in ${ }_{\Delta_{G}} \mathcal{C}\left(G^{2}\right)_{\Delta_{G}}^{\tilde{\omega}}$ is the dimension of its total space divided by $\left.|G|\right)$. Thus $\mathcal{D}_{\omega}(K)$ has the same global dimension as the MTC of local $A_{\Delta}$-modules, of which it is a full subcategory, and hence the two must be equal. The map $\iota$ ! of Theorem 1(b)(iii) sends $[k, \bar{\chi}]$ to $\left[k, \operatorname{Ind}_{C_{K}(k)}^{C_{G}(k)} \bar{\chi}\right]$.

\subsection{Proof of type 1-ness in general case}

The nesting of type 1 module categories is developed in Proposition 4.16 of [35] and Proposition 2.3.2 of [17]; we summarise it here. Suppose $\mathcal{M}_{1}$ is a type 1 module category for an MTC $\mathcal{C}$, and let $\mathcal{C}_{1}$ be its MTC of local modules. Suppose $\mathcal{M}_{2}$ is a type 1 module category for $\mathcal{C}_{1}$, let $A_{2}$ be the associated commutative algebra, and let $\mathcal{C}_{2}$ be its MTC of local modules. Then $A_{2}$ and indeed all of $\mathcal{C}_{2}$ are objects in $\mathcal{C}_{1}$ (this map is the forgetful functor called sigma-restriction). Likewise, every object in $\mathcal{C}_{1}$ is an object in $\mathcal{C}$. Let $A$ be the algebra $A_{2}$ regarded as an object in $\mathcal{C}$ in this sense. $A$ is an algebra in $\mathcal{C}$ : the multiplication $\mu_{A}: A \otimes_{\mathcal{C}} A \rightarrow A$ is the composition of the projection to the fusion product $A_{2} \otimes_{\mathcal{C}_{1}} A_{2}$ in $\mathcal{C}_{1}$, with the multiplication $\mu_{A_{2}}: A_{2} \otimes_{\mathcal{C}_{1}} A_{2} \rightarrow A_{2}$, which we identify with (sigma-restrict to) $A$ ( $V \otimes_{\mathcal{C}_{1}} W$ is naturally a quotient of $V \otimes_{\mathcal{C}} W$ - see Theorem 1.5 of [53]). The corresponding module category $\mathcal{M}$ of $A$-modules in $\mathcal{C}$ is the desired nesting or transfer of $\mathcal{M}_{2}$ to $\mathcal{C}$ via $\mathcal{M}_{1}$. A will be type 1 when both $\mathcal{M}_{1}$ and $\mathcal{M}_{2}$ are type 1 . The MTC of local modules for $\mathcal{M}$ is the sigma-restriction of $\mathcal{C}_{2}$ first to $\mathcal{C}_{1}$ then to $\mathcal{C}$.

Consider now type $1 N$, so $H=\Delta_{G}(1 \times N)$. The algebra $A_{N}=\theta_{N}$ here is the bundle in ${ }_{\Delta_{G}} \mathcal{C}\left(G^{2}\right)_{H}^{\tilde{\omega}, \psi}$ (hence ${ }_{\Delta_{G}} \mathcal{C}\left(G^{2}\right)_{\Delta_{G}}^{\tilde{\omega}}$ ) consisting of $\mathbb{C}$ attached to each point in $H$, with a $\Delta_{G}^{L} \times H^{R}$-action given by (2.16)-(2.18), where the fibre $\mathbb{C}_{(1,1)}$ has the trivial representation of the stabiliser $\Delta_{G}^{a d j}$. The algebra multiplication $\mu_{N}: \mathbb{C}_{(1,1)} \otimes \mathbb{C}_{(n, 1)} \rightarrow$ $\mathbb{C}_{(n, 1)}$ is that of $\mathbb{C}$; to obtain the product $\mu_{N}: \mathbb{C}_{(g n, g)} \otimes \mathbb{C}_{\left(g^{\prime} n^{\prime}, g^{\prime}\right)} \rightarrow \mathbb{C}_{\left(g g^{\prime} n^{\prime} n^{\prime}, g g^{\prime}\right)}$, use equivariance (3.4) as before. Then $A_{N}$ decomposes into a sum of $\mathcal{D}_{\omega}(G)$ sectors as $A_{N}=\sum_{n}\left[(n, 1), \beta_{(n, 1)}\right]$, where the sum is over representatives $n$ of each $G$-conjugacy class (i.e. $G^{a d j}$-orbit) in $N$. A bundle in ${ }_{\Delta_{G}} \mathcal{C}\left(G^{2}\right)_{\Delta_{G}}^{\tilde{\tilde{a}}}$ is an $A_{N}$-module, iff it is also

a bundle in ${ }_{\Delta_{G}} \mathcal{C}\left(G^{2}\right)_{H}^{\tilde{\omega}, \psi}$. The indecomposable right $A_{N}$-modules in ${ }_{\Delta_{G}} \mathcal{C}\left(G^{2}\right)_{\Delta_{G}}^{\tilde{\omega}}$ are precisely the indecomposable bundles in ${ }_{\Delta_{G}} \mathcal{C}\left(G^{2}\right)_{H}^{\tilde{\omega}, \psi}$ with $\Delta_{G}^{L} \times H^{R}$ equivariance restricted to $\Delta_{G}^{L \times R}$. Since the fibre of $A_{N}$ above $(1,1) \in G^{2}$ is a commutative algebra, this is also type 1 .

Finally, consider the general case, $H=\Delta_{K}(1 \times N)$ with $\psi$ as in Theorem 1 . We know from last subsection that $\left(\Delta_{K}, 1\right)$ is a type 1 (in fact type $1 \Delta$ ) module category $\mathcal{M}_{1}$ for $\mathcal{D}_{\omega}(G)$, whose MTC of local modules is $\mathcal{D}_{\omega}(K)$. We know from the previous paragraph that $\left(\Delta_{K}(1 \times N), \psi\right)$ is a type 1 module category (in fact type $1 N)$ for $\mathcal{D}_{\omega}(K)$. Nesting these, we obtain the module category $\left(\Delta_{K}(1 \times N), \psi\right)$ of $\mathcal{D}_{\omega}(G)$, which is therefore type 1 . The description of sigma-restriction last subsection makes it easy to describe the algebra and MTC of local modules for this nesting. In 
particular, the algebra $A_{N}$ as a bundle in ${ }_{\Delta_{K}} \mathcal{C}\left(K^{2}\right)_{\Delta_{K}}^{\tilde{\omega}}$ consists of a copy of $\mathbb{C}$ above each point in $\Delta_{K}(1 \times N)$; it is the identical bundle in ${ }_{\Delta_{K}} \mathcal{C}\left(G^{2}\right)_{\Delta_{K}}^{\tilde{\omega}}$; it corresponds to the bundle in ${ }_{\Delta_{G}} \mathcal{C}\left(G^{2}\right)_{\Delta_{K}}^{\tilde{\omega}}$ consisting of a copy of $\mathbb{C}$ (carrying the representation $\left.\beta_{(1, n)}\right)$ above every point in $\Delta_{G}(1 \times N)$; and finally it corresponds to the bundle in $\Delta_{G} \mathcal{C}\left(G^{2}\right)_{\Delta_{G}}^{\tilde{\omega}}$ described in section 3.1 .

This shows that the pairs $(H, \psi)$ described in the second sentence of Theorem 1(a) are indeed type 1 . The converse is Theorem 3.15 of [19], though it also falls out from the treatment of general module categories for $\mathcal{D}_{\omega}(G)$ which we develop in [31]. The final sentence of Theorem 1(a) is deferred to after Claim 2, in section 4.7.

\subsection{Proof of alpha-induction}

In a type 1 theory, alpha-induction can be defined purely categorically, in terms of the algebra $A$ [53], as we sketched in section 2.1: we get a functor $\alpha^{H}$ from $\mathcal{D}_{\omega}(G)$ to right $A_{H}$-modules ${ }_{\Delta_{G}} \mathcal{C}\left(G^{2}\right)_{H}^{\tilde{\omega}, \psi}$, or functors $\alpha_{ \pm}^{H}$ to $A_{H}-A_{H}$-bimodules ${ }_{H}^{\psi} \mathcal{C}\left(G^{2}\right)_{H}^{\tilde{\omega}, \psi}$. The more fundamental is $\alpha^{H}$; we revisit $\alpha_{ \pm}^{H}$ next subsection when we compute the branching rules. Unlike sigma-restriction of local modules, alpha-induction for a nesting is not simply a composition of the alpha-inductions for each component, but we have learned enough that it is now straightforward to do it in one step.

Consider any $\left(\Delta_{K}(1 \times N), \psi\right)$ as in Theorem 1(a). As is clear from the previous two subsections, its commutative algebra is $A_{H}=\sum_{n}\left[(n, 1), \operatorname{Ind}_{C_{K}(n)}^{C_{G}(n)} \beta_{(n, 1)}^{*}\right]$, where the sum is over representatives of the $K^{a d j}$ orbits in $N$. Note that $A_{\Delta}=\left[(1,1), \operatorname{Ind}_{K}^{G} 1\right]$ is a subalgebra of $A_{H}$, and so an $A_{H}$-module is also an $A_{\Delta}$-module. In fact, a bundle in ${ }_{\Delta_{G}} \mathcal{C}\left(G^{2}\right)_{H}^{\tilde{\omega}, \psi}$ is an $A_{H}$-module iff it is an $A_{\Delta}$-module, hence expressible in the form $\sum\left[g, \operatorname{Ind}_{C_{K}(g)}^{C_{G}(g)} \bar{\chi}\right]$, and also the corresponding bundle $\sum[(g, 1), \bar{\chi}] \in{ }_{\Delta_{G}} \mathcal{C}\left(G^{2}\right)_{\Delta_{K}}^{\tilde{\omega}}$ is also a bundle in ${ }_{\Delta_{G}} \mathcal{C}\left(G^{2}\right)_{H}^{\tilde{\omega}, \psi}$ (though with $\Delta_{G}^{L} \times H^{R}$-equivariance restricted to $\Delta_{G}^{L} \times \Delta_{K}^{R}$ ). This association of any bundle in ${ }_{\Delta_{G}} \mathcal{C}\left(G^{2}\right)_{H}^{\tilde{\omega}, \psi}$ to some bundle in ${ }_{\Delta_{G}} \mathcal{C}\left(G^{2}\right)_{\Delta_{G}}^{\tilde{\omega}}$ is sigmarestriction here.

Then alpha-induction sends a sector $[(g, 1), \chi]$ to the bundle product $[(g, 1), \chi] \otimes$ $A_{H}$. Let's compute this from the intermediate point, i.e. as a bundle in ${ }_{\Delta_{G}} \mathcal{C}\left(G^{2}\right)_{\Delta_{K}}$ : from (2.20) we obtain

$$
\begin{aligned}
\alpha^{H}([g, \chi]) & \leftrightarrow \sum_{n}[(g, 1), \chi] \otimes_{\Delta_{G}}\left[(n, 1), \beta_{(n, 1)}^{*}\right] \\
& =\sum_{n} \sum_{h \in C_{G}(g) \backslash G / C_{K}(n)}\left[\left(g^{h} n, 1\right), \operatorname{Ind}_{C_{K}(n) \cap C_{K}\left(g^{h} n\right)}^{C_{K}\left(g^{h} n\right)}\left(\chi^{h n} \beta_{(n, 1)}^{*}\right)\right],
\end{aligned}
$$

where we use the $\Delta_{G}^{L}$ action to choose more convenient orbit representatives. Interpreting this as a bundle in ${ }_{{ }_{G}} \mathcal{C}\left(G^{2}\right)_{H}^{\tilde{\omega}, \psi}$ is the same as dropping the sum over $n$, so we obtain (3.2).

As a consistency check, the algebra $A^{H}$ can be identified with the bundle $\iota=$ $[(1,1), \mathbf{1}]$ in ${ }_{\Delta_{G}} \mathcal{C}\left(G^{2}\right)_{H}^{\tilde{\omega}, \psi}$, as discussed in [26]. Its transpose $\bar{\iota}$ is the bundle $[(1,1), \mathbf{1}]$ in ${ }_{H}^{\psi} \mathcal{C}\left(G^{2}\right)_{\Delta_{G}}^{\tilde{\omega}}$. The sigma-restriction functor from the full system ${ }_{H}^{\psi} \mathcal{C}\left(G^{2}\right)_{H}^{\tilde{\omega}, \psi}$ to $\mathcal{D}_{\omega}(G)$, can be computed as the product $\iota a \bar{\iota}$ of bundles. 


\subsection{Local modules and branching rules}

In this subsection we prove Theorem 1(b)(ii), i.e. we work out explicitly sigmarestriction ${ }_{\Delta_{G}} \mathcal{C}\left(G^{2}\right)_{H}^{\tilde{\omega}, \psi} \rightarrow{ }_{\Delta_{G}} \mathcal{C}\left(G^{2}\right)_{\Delta_{G}}^{\tilde{\omega}}$, and identify the local bundles in ${ }_{\Delta_{G}} \mathcal{C}\left(G^{2}\right)_{H}^{\tilde{\omega}, \psi}$.

The type $1 \Delta$ case, i.e $\left(\Delta_{K}, 1\right)$, is worked out in section 4.1. In particular, the MTC of local modules is $\mathcal{D}_{\omega}(K)$, and sigma-restriction $\operatorname{Res} \operatorname{Ver}_{\omega}(K) \rightarrow \operatorname{Ver}_{\omega}(G)$ of local modules is $\operatorname{Res}([k, \bar{\chi}])=\left[k, \operatorname{Ind}_{C_{K}(k)}^{C_{G}(k)} \bar{\chi}\right]$, where $\bar{\chi} \in \operatorname{Irr}_{c_{k}}\left(C_{K}(k)\right)$ and Ind is induction to a $c_{k}$-projective $G$-character. Of course this coincides with the $K$-theoretic map $\iota$ ! where $\iota$ is the embedding $K \hookrightarrow G$, hence it coincides with Theorem 1(b)(iii) in this special case.

Now turn to the type $1 N$ case, i.e. $H=\Delta_{G}(1 \times N)$ and $\psi$ as in Theorem 1. Choose any indecomposable bundle $[(k, 1), \chi]$ in ${ }_{\Delta_{G}} \mathcal{C}\left(G^{2}\right)_{H}^{\tilde{\omega}, \psi}$. Its support is $\Delta_{G}(k, 1) H=$ $(\operatorname{cl}(k) \times 1) H$ where $\operatorname{cl}(k)$ is the conjugacy class of $k$ in $G$. The stabiliser of $(k, 1)$ is $\operatorname{St}_{H}(k, 1)=\left\{\left(h_{+}, h_{-}\right) \in H: h_{-} k=k h_{+}\right\} \cong C_{H}(k)$. As we know, this bundle sigmarestricts to a bundle in ${ }_{\Delta_{G}} \mathcal{C}\left(G^{2}\right)_{\Delta_{G}}^{\tilde{\omega}}$ simply by restricting $\Delta_{G}^{L} \times H^{R}$ equivariance to $\Delta_{G}^{L \times R}$. If we write the set $\operatorname{cl}(k) N$ as a disjoint union $\cup_{n} \operatorname{cl}(k n)$ over certain $n \in N$ (possible, since $N$ is normal in $G$ ), this sigma-restriction becomes $\sum_{n}\left[(k n, 1), \chi^{\prime}\right]$, where $\chi^{\prime}$ is the appropriate character (identified shortly) of the stabiliser $\operatorname{St}_{H}(k n, 1) \cap$ $\Delta_{G} \cong C_{G}(k n)$.

To identify that character $\chi^{\prime}$, let $V$ be the fibre above $(k, 1)$ in the bundle $[(k, 1), \chi]$, carrying a $C_{H}(k)$-irrep $\rho$ realising the character $\chi$. Choose any $n \in N$ and let $V^{\prime}$ be the fibre above $(k n, 1)$ in that bundle; we can identify $V^{\prime}$ with $V$ through the invertible map $v \mapsto v^{\prime}=v \cdot(n, 1)$. Note that $\left(g, g^{\prime}\right) \in \operatorname{St}_{H}(k n, 1)$ iff $\left({ }^{n} g, g^{\prime}\right) \in \mathrm{St}_{H}(k, 1)$. We can compare $\rho^{\prime}\left(g, g^{\prime}\right) v^{\prime}:=\left(g^{\prime}, g^{\prime}\right) \cdot\left(v^{\prime} \cdot\left(g, g^{\prime}\right)^{-1}\right) \in V^{\prime}$ to $\rho\left({ }^{n} g, g^{\prime}\right) v:=\left(g^{\prime}, g^{\prime}\right) \cdot\left(v \cdot\left({ }^{n} g, g^{\prime}\right)^{-1}\right)$ through the moves $\left(g^{\prime}, g^{\prime}\right) \cdot\left((v \cdot(n, 1)) \cdot\left(g, g^{\prime}\right)^{-1}\right) \approx$ $\left(g^{\prime}, g^{\prime}\right) \cdot\left(v \cdot\left(n g^{-1}, g^{\prime-1}\right)\right)=\left(g^{\prime}, g^{\prime}\right) \cdot\left(v \cdot\left(\left({ }^{n} g, g^{\prime}\right)^{-1}(n, 1)\right)\right) \approx\left(\left(g^{\prime}, g^{\prime}\right) \cdot\left(v \cdot\left({ }^{n} g, g^{\prime}\right)^{-1}\right)\right) \cdot(n, 1)$, where ' $\approx$ ' means equality up to a phase from (2.16)-(2.18); keeping track of the phases, we obtain

$$
\rho^{\prime}\left(g, g^{\prime}\right) v^{\prime}=\beta_{(n, 1)}\left({ }^{n} g^{-1}, g^{\prime-1}\right)^{*} \omega\left(k, n, g^{-1}\right)^{*} \omega\left(k,{ }^{n} g^{-1}, n\right) \omega\left(g^{\prime}, g^{\prime-1} k, n\right)\left(\rho\left({ }^{n} g, g^{\prime}\right) v\right)^{\prime}
$$

Again, $\rho^{\prime}$ is the representation of $\operatorname{St}_{H}(k n, 1)$ in the fibre above $(k n, 1)$ in the bundle $[(k, 1), \chi]$; we are interested in its restriction $\chi^{\prime}$ to $\operatorname{St}_{H}(k n, 1) \cap \Delta_{G} \cong C_{G}(k n)$.

We want to identify the local $A^{H}$-modules, i.e. the indecomposable bundles $[(k, 1), \chi]$ in ${ }_{\Delta_{G}} \mathcal{C}\left(G^{2}\right)_{H}^{\tilde{\omega}, \psi}$ which, when we restrict to a bundle in ${ }_{\Delta_{G}} \mathcal{C}\left(G^{2}\right)_{\Delta_{G}}^{\tilde{\omega}}$, has a well-defined twist. Choose any $n \in N$ and $g \in C_{G}(k n)$; then $(g, g) \in \operatorname{St}_{H}(k n, 1)$. Writing $\Delta_{h}=(h, h)$, the twist (or $T$-matrix eigenvalue) of the indecomposable bundle $\left[\left(k^{\prime}, 1\right), \chi^{\prime}\right]$ in ${ }_{\Delta_{G}} \mathcal{C}\left(G^{2}\right)_{\Delta_{G}}^{\tilde{\omega}_{G}} \cong \mathcal{D}_{\omega}(G)$ is $\chi^{\prime}\left(k^{\prime}\right) / \chi^{\prime}(1)$. So the bundle $[(k, 1), \chi]$ in ${ }_{\Delta_{G}} \mathcal{C}\left(G^{2}\right)_{H}^{\tilde{\omega}, \psi}$ is local iff $\chi(k, k) / \chi(1,1)$ has modulus 1 (i.e. $\rho(k, k)$ is scalar), and

$\chi(k, k)=\beta_{(n, 1)}\left(k^{-1} n^{-1}, n^{-1} k^{-1}\right)^{*} \omega\left(k, n, n^{-1} k^{-1}\right)^{*} \omega\left(k, k^{-1} n^{-1}, n\right) \omega\left(k n, n^{-1}, n\right) \chi(n k, k n)$

for all $n \in N$. Note that some characters $\chi$ of $\operatorname{St}_{H}(k, 1)$ may not satisfy $|\chi(k, k)|=$ $\chi(1,1)$, since $(k, k)$ will not in general lie in the centre of $\mathrm{St}_{H}(k, 1)$. 
Sigma-restriction and local modules for general type $1(H, \psi)$ is now a straightforward nesting of the treatments for types $1 \Delta$ and $1 N$, and is as in Theorem 1 (b)(ii).

\subsection{The associated modular tensor category}

In this subsection we identify the MTC of local modules as a twisted group double.

The type $1 \Delta$ case was worked out in section 4.1 , so consider first type $1 N$, i.e. $H=\Delta_{G}(1 \times N)$ and $\psi$ as in Theorem 1 . We will find that the effect of the 2-cocycle $\psi$ is surprisingly subtle. Write $\bar{G}:=G / N$ and $\pi: G \rightarrow \bar{G}$ for the corresponding homomorphism. In the following, we identify $C_{\Delta_{G}}(n g, 1)$ with $C_{G}(n g)$ and $C_{H}(g) / N$ with $C_{\bar{G}}(g N)$.

We begin with the elementary observation that a projective representation of a group $K$ defines a projective representation for $G / N, N$ the projective kernel:

Claim 1. Let $N$ be a normal subgroup of $K$, and fix any transversal $t_{\bar{k}}$ for $K / N$. Let $c \in Z^{2}(K ; \mathbb{T})$ be normalised.

(a) Suppose $\chi \in \operatorname{Irr}_{c}(K)$ satisfies $|\chi(n)|=\chi(1)$ for all $n \in N$. Then $\phi(n):=$ $\chi(n) / \chi(1)$ satisfies

$$
\phi(n) \phi\left(n^{\prime}\right)=c\left(n, n^{\prime}\right) \phi\left(n n^{\prime}\right), \phi\left(n^{k}\right) c(n, k)=\phi(n) c\left(k, n^{k}\right) \forall n, n^{\prime} \in N, k \in K .
$$

(b) Suppose $\phi: N \rightarrow \mathbb{T}$ satisfies (4.4), and define

$$
\bar{c}(\bar{h}, \bar{k}):=\phi\left(t_{\bar{h}} t_{\bar{k}} t_{\bar{h} \bar{k}}^{-1}\right) c\left(t_{\bar{h}}, t_{\bar{k}}\right) c\left(t_{\bar{h}} t_{\bar{k}} t_{\bar{h} \bar{k}}^{-1}, t_{\bar{h} \bar{k}}\right)^{*} .
$$

Then the relation $\bar{\chi}(\bar{k})=\chi\left(t_{\bar{k}}\right)$ defines a bijection between the set of $\chi \in \operatorname{Irr}_{c}(K)$ satisfying $\chi(n)=\phi(n) \chi(1)$ for all $n \in N$, and $\bar{\chi} \in \operatorname{Irr}_{\bar{c}}(K / N)$.

Proof. Part (a) is trivial: let $\rho$ be the projective representation associated to $\chi$, and compute $\rho(n) \rho\left(n^{\prime}\right)$ and $\rho(k) \rho(n)$.

To prove (b), first let $\chi$ be as above, again realised by $\rho$. First note that the matrices $\rho(n)$ are scalar for $n \in N$ (this follows from the triangle inequality applied to $\chi(n)=\phi(n) \chi(1))$. Now define $\bar{\rho}(\bar{h})=\rho\left(t_{\bar{h}}\right)$. Then for all $\bar{h}, \bar{k} \in K / N$,

$$
\bar{\rho}(\bar{h}) \bar{\rho}(\bar{k})=c\left(t_{\bar{h}}, t_{\bar{k}}\right) \rho\left(t_{\bar{h}} t_{\bar{k}}\right)=c\left(t_{\bar{h}}, t_{\bar{k}}\right) c\left(t_{\bar{h}} t_{\bar{k}} t_{\bar{h} \bar{k}}^{-1}, t_{\bar{h} \bar{k}}\right)^{*} \rho\left(t_{\bar{h}} t_{\bar{k}} t_{\bar{h} \bar{k}}^{-1}\right) \rho\left(t_{\bar{h} \bar{k}}\right)=\bar{c}(\bar{h}, \bar{k}) \bar{\rho}(\bar{h} \bar{k})
$$

as desired. Conversely, given $\bar{\chi} \in \operatorname{Irr}_{\bar{c}}(K / N)$, define $\chi\left(n t_{\bar{k}}\right)=c\left(n, t_{\bar{k}}\right) \phi(n) \bar{\chi}(\bar{k})$ for all $n \in N, \bar{k} \in K / N$, then the same calculation shows $\chi \in \operatorname{Irr}_{c}(K)$. Furthermore, these two maps $\chi \mapsto \bar{\chi}$ and $\bar{\chi} \mapsto \chi$ are inverses. $\quad$ QED to Claim 1

Consider any sector $[g, \chi] \in \operatorname{Ver}_{\omega}(G)$ appearing in the sigma-restriction of a local bundle in ${ }_{\Delta_{G}} \mathcal{C}\left(G^{2}\right)_{H}^{\tilde{\omega}, \psi}$. Then Claim 1 with $K$ there replaced with $C_{H}(g), c$ replaced with $c_{g}$, and $\phi$ obtained from (4.3), tells us that the $\chi \in \operatorname{Irr}_{c_{g}}\left(C_{H}(g)\right)$ satisfying (4.3) are in natural bijection with the $\bar{\chi} \in \operatorname{Irr}_{\bar{c}_{g}}\left(C_{\bar{G}}(\bar{g})\right)$, where $\bar{c}_{g}=\bar{c}_{g}^{\omega} \bar{c}_{g}^{\psi}$ for

$$
\begin{aligned}
\bar{c}_{g}^{\omega}(\bar{h}, \bar{k}) & =\omega(n g, n, g)^{*} \omega(n, n g, g) \omega\left(n, n g^{2}, g^{-1} n^{-1}\right) c_{g}(n, g) c_{g}\left(t_{\bar{h}}, t_{\bar{k}}\right) c_{g}\left(n, t_{\bar{h} \bar{k}}\right)^{*} \\
\bar{c}_{g}^{\psi}(\bar{h}, \bar{k}) & =\beta_{(g, g)}(n, 1)
\end{aligned}
$$


and where we write $n=t_{\bar{h}} t_{\bar{k}} t_{\bar{h} \bar{k}}^{-1}$.

Note that the 2-cocycle $\psi$ and the 3-cocycle $\omega$ are completely independent: the pair $(H, \psi)$ is type 1 for $\mathcal{D}_{\omega}(G)$, iff $(H, 1)$ is type 1 for $\mathcal{D}_{\omega}(G)$; moreover, in that case it is also type 1 for $\mathcal{D}_{1}(G)$. In addition, in (4.5),(4.6) we factorised the multiplier $\bar{c}_{g}=\bar{c}_{g}^{\omega} \bar{c}_{g}^{\psi}$ where $\bar{c}_{g}^{\omega}$ depends on $\omega$ but not $\psi$, and $\bar{c}_{g}^{\psi}$ depends on $\psi$ but not $\omega$.

We want to find a 3-cocycle on $\bar{G}$ which is responsible (in the sense of (2.5)) for these multipliers $\bar{c}_{g}$. The preceding paragraph means that it (if it exists) will factorise into a part $\bar{\omega}$ seeing only $\omega$, and a part $\bar{\omega}^{\psi}$ seeing only $\psi$.

Fix a transversal $t_{\bar{g}}$ for $G / N$. Recall that $\left.\widetilde{\omega}\right|_{H}$ must be coboundary.

Proposition 2. $[19,47]$ Let $F$ be a 2-cochain on $H$ such that $d F=\left.\widetilde{\omega}\right|_{H}$. Define

$$
\begin{array}{r}
\bar{\omega}(\bar{g}, \bar{h}, \bar{k})=\omega\left(k_{1}, t_{\bar{h}}, t_{\bar{k}}\right) \\
F\left(\left(n_{1}, 1\right),\left(n_{2}, 1\right)\right) F\left(\left(n_{3}, 1\right),\left(n_{2} n_{1}, 1\right)\right) \times \\
F\left(\left(n_{3}^{-1}, 1\right),\left(n_{3}, 1\right)\right) \beta_{\left(k_{1}, k_{1}\right)}^{F}\left(n_{4}, 1\right) \\
\bar{\omega}^{\psi}(\bar{g}, \bar{h}, \bar{k})=\psi\left(\left(n_{2}, 1\right),\left(n_{1}, 1\right)\right) \psi\left(\left(n_{4}^{-1}, 1\right),\left(n_{5}, 1\right)\right)^{*} \beta_{\left(t_{\bar{g}}, t_{\bar{g}}\right)}\left(n_{4}^{-1}, 1\right),
\end{array}
$$

where $k_{1}=t_{\bar{g} \bar{h} \bar{k}} t_{\bar{k}}^{-1} t_{\bar{h}}^{-1}, n_{1}=t_{\bar{g} \bar{h}} t_{\bar{k}} t_{\bar{g} \bar{h} \bar{k}}^{-1}, n_{2}=t_{\bar{g}} t_{\bar{h}} t_{\bar{g} \bar{h}}^{-1}, n_{4}=t_{\bar{h}} t_{\bar{k}} t_{\bar{h} \bar{k}}^{-1}, n_{3}=k_{1}\left(n_{4}^{-1}\right)$, $n_{5}=t_{\bar{g}} t_{\bar{h} \bar{k}} t_{\bar{g} \bar{h} \bar{k}}^{-1}$, and where $\beta^{F}$ is as in (2.7). Then both $\bar{\omega}, \bar{\omega}^{\psi} \in Z^{3}(\bar{G} ; \mathbb{T})$ and are normalised, and when substituted into (2.5) recover $\bar{c}_{g}^{\omega}$ and $\bar{c}_{g}^{\psi}$ respectively.

Choosing a different transversal $t_{\star}$ won't change the cohomology class of the 3cocycles. Up to notational differences, (4.7) agrees with equation (20) in [19] when the 2-cocycle $\psi$ is taken to be 1 . The calculation of $\bar{\omega}^{\psi}$ is also contained in [19] (when $\omega=1$ ), but since we will later need to exploit more structure (see especially section 4.9) we prefer the treatment in [47], even though it necessitates chopping the calculation of the 3 -cocycle into the two parts. The treatments are equivalent, indeed the explicit calculations given in the Theorem 2.17 proof would make equal sense in [47]. It would be nice though to understand conceptually why the two contexts are equivalent in this sense. The argument that these 3-cocycles recover the appropriate 2-cocycles follows from [19].

The source of (4.8) is the thesis of Vaughan Jones [47]. Jones verified in section 4.2 of [47], that for any 2-cocycle $\mu \in Z^{2}(N ; \mathbb{T})$ and map $\lambda: G \times N \rightarrow \mathbb{T}$ satisfying the following equations

$$
\begin{aligned}
\lambda(m, n) & =\mu\left(m, n^{m}\right) \mu(n, m)^{*}, \\
\lambda(g h, n) & =\lambda(g, n) \lambda\left(h, n^{g}\right), \\
\lambda(g, m n) \lambda(g, m)^{*} \lambda(g, n)^{*} & =\mu(m, n) \mu\left(m^{g}, n^{g}\right)^{*}, \\
\lambda(1, n)=\lambda(g, 1) & =\mu(1, n)=\mu(n, 1)=1,
\end{aligned}
$$

for all $m, n \in N, g, h \in G$, the map $\bar{\omega}^{\psi}$ is indeed a 3-cocycle on $\bar{G}$. Now, $\lambda(g, n)=\beta_{g}^{\prime}(n)$ and $\mu(m, n)=\psi^{\prime}(m, n)$ satisfy these equations: (4.9)-(4.11) is (2.7)(2.9) respectively, and (4.12) follows because $\psi$ is normalised, so we can use Jones' result.

Jones was interested in determining complete invariants for actions of finite groups $G$ on the hyperfinite $\mathrm{II}_{1}$ factor. His invariants consist of a normal subgroup $N$, and 
$\lambda, \mu$ satisfying these equations. A nice review of this circle of ideas is [70]. Incidentally, in section 4.9 we need the converse, that any solution $\lambda, \mu$ comes from a $\psi$ in this way. So this point of contact between our context and that of Jones is pronounced.

Inflating, in the usual sense, an indecomposable bundle in ${ }_{\Delta_{\bar{G}}} \mathcal{C}\left(\bar{G}^{2}\right)_{\tilde{\Delta}_{\bar{G}}^{\prime}}$ (where we write $\omega^{\prime}=\overline{\omega \omega}^{\psi}$ ) gives a (local) indecomposable bundle in ${ }_{\Delta_{G}} \mathcal{C}\left(G^{2}\right)_{\Delta_{G}}^{\tilde{\omega}}$. The equivalence of the category of local modules in ${ }_{\Delta_{G}} \mathcal{C}\left(G^{2}\right)_{H}^{\tilde{\omega}, \psi}$ and $\mathcal{D}_{\bar{\omega}^{\prime}}(\bar{G})$ for the appropriate $\bar{\omega}^{\prime}$ is established in Theorem 2.17 of [19].

Sigma-restriction $\operatorname{Ver}_{\bar{\omega} \bar{\omega}^{\psi}}(\bar{G}) \rightarrow \operatorname{Ver}_{\omega}(G)$ is the map $\pi^{*}$ of Theorem 1(b)(iii) corresponding to the group homomorphism $\pi: G \rightarrow \bar{G}$. We can read off from the proof of Claim 1(b) that an indecomposable bundle $[\bar{g}, \bar{\chi}] \in \operatorname{Ver}_{\bar{\omega} \bar{\omega}^{\psi}}(\bar{G})$ is sent to

$$
[\bar{g}, \bar{\chi}] \mapsto \sum_{g}[g, \widetilde{\phi} \bar{\chi} \circ \pi]
$$

where the sum over $g$ is over representatives of the distinct conjugacy classes in $G$ projecting to the $\bar{G}$-conjugacy class of $\bar{g}$, and $\widetilde{\phi}(h)=c_{g}\left(h t_{h N}^{-1}, t_{h N}\right) \phi\left(h t_{h N}^{-1}\right)$ where $\phi$ is as in (4.3). As explained in the previous paragraph, the quantities $\widetilde{\phi} \bar{\chi} \circ \pi_{g}$ can be identified with $c_{g}^{\omega}$-projective characters of $C_{G}(g)$ behaving appropriately with respect to $n \in C_{N}(g)$, as (4.3) requires.

The general type 1 module category $\left(\Delta_{K}(1 \times N), \psi\right)$ is a combination of the type $1 \Delta$ pair $\left(\Delta_{K}, 1\right)$ followed by the type $1 N$ pair $\left(\Delta_{K}(1 \times N), \psi\right)$. Then the MTC of local bundles for the desired type 1 module category, as well as sigma-restriction from local bundles to $\operatorname{Vec}_{\omega}(G)$, is obtained from the type $1 N$ and $1 \Delta$ ones as explained in the beginning of section 4.2. The result is given in Theorem 1(b)(iii).

\subsection{Proof of Corollary 1}

Take $G=\langle g\rangle \cong \mathbb{Z}_{n}$. Write $m=|K|$ and $m^{\prime}=|N|$. Then $m^{\prime}|m| n$ and $K=\left\langle g^{n / m}\right\rangle$, $N=\left\langle g^{n / m^{\prime}}\right\rangle$, and $H=\Delta_{K}(1 \times N) \cong \mathbb{Z}_{m^{\prime}} \times \mathbb{Z}_{m}$, where the $\mathbb{Z}_{m^{\prime}}$ factor has generator $g_{1}=\left(g^{n / m^{\prime}}, 0\right)$ and the $\mathbb{Z}_{m}$ factor has generator $g_{2}=\left(g^{n / m}, g^{n / m}\right)$.

Explicit $k$-cocycles are worked out in [41] for all abelian groups and all $k$. For the group $H \cong \mathbb{Z}_{m^{\prime}} \times \mathbb{Z}_{m}, H^{3}(H ; \mathbb{T}) \cong \mathbb{Z}_{m^{\prime}}^{2} \times \mathbb{Z}_{m}$ with representatives

$$
\begin{aligned}
\omega_{a_{1}, a_{2}, a_{12}}\left(g_{1}^{i_{1}} g_{2}^{i_{2}}, g_{1}^{j_{1}} g_{2}^{j_{2}}, g_{1}^{k_{1}} g_{2}^{k_{2}}\right)= & \exp \left(2 \pi i \frac{a_{1} i_{1}}{m^{\prime}}\left[\frac{j_{1}+k_{1}}{m^{\prime}}\right]\right) \times \\
& \exp \left(2 \pi i \frac{a_{2} i_{2}}{m}\left[\frac{j_{2}+k_{2}}{m}\right]\right) \exp \left(2 \pi i \frac{a_{12} i_{2}}{m^{\prime}}\left[\frac{j_{1}+k_{1}}{m^{\prime}}\right]\right)
\end{aligned}
$$

for integers $0 \leq a_{1}, a_{12}<m^{\prime}$ and $0 \leq a_{2}<m$. Now $\left.\widetilde{\omega}_{q}\right|_{H}$ is the 3 -cocycle given by

$\widetilde{\omega}_{q}\left(g_{1}^{i_{1}} g_{2}^{i_{2}}, g_{1}^{j_{1}} g_{2}^{j_{2}}, g_{1}^{k_{1}} g_{2}^{k_{2}}\right)=\exp \left(2 \pi i \frac{q}{m}\left(\frac{m}{m^{\prime}} i_{1}+i_{2}\right)\left[\frac{j_{1}+k_{1}}{m^{\prime}}+\frac{j_{2}+k_{2}}{m}\right]\right) \exp \left(-2 \pi i \frac{q}{m} i_{2}\left[\frac{j_{2}+k_{2}}{m}\right]\right)$

This is required to be coboundary, so in particular the restriction to the subgroup $\left\langle g_{1}\right\rangle \cong \mathbb{Z}_{m^{\prime}}$ must be coboundary, but that restriction is clearly $q \omega_{1}^{\prime}$ where $\omega_{1}^{\prime}$ is the 
generator of $H^{3}\left(\mathbb{Z}_{m^{\prime}} ; \mathbb{T}\right) \cong \mathbb{Z}_{m^{\prime}}$ given in (2.13). This restriction is coboundary iff $m^{\prime} \mid q$. Write $\left.\left[\tilde{\omega}_{1}\right]\right|_{H}=\left[\omega_{a_{1}, a_{2}, a_{12}}\right]$. Since the restriction of $\left.\tilde{\omega}_{1}\right|_{H}$ to $\Delta_{K}$ is coboundary (in fact identically 1 ), $a_{2}=0$. Therefore $\left.\left[\tilde{\omega}_{1}\right]\right|_{H}=\left[\omega_{a_{1}, 0, a_{12}}\right]$ has order dividing $m^{\prime}$, so $\left.\left[\tilde{\omega}_{q}\right]\right|_{H}=\left.q\left[\tilde{\omega}_{1}\right]\right|_{H}=[1]$. Hence the only conditions on $H$ (i.e. on $m^{\prime}, m$ ) are $m^{\prime}|m| n$ and $m^{\prime} \mid q$.

By the Künneth formula, $H^{2}(H ; \mathbb{T}) \cong \mathbb{Z}_{m^{\prime}}$. We find (e.g. [41]) that $\psi$ can be taken to be

$$
\psi_{a}\left(g_{1}^{i_{1}} g_{2}^{i_{2}}, g_{1}^{j_{1}} g_{2}^{j_{2}}\right)=\exp \left(a i_{1} j_{2} / m^{\prime}\right)
$$

where $0 \leq a<m^{\prime}$. Since $H^{2}\left(\mathbb{Z}_{m} ; \mathbb{T}\right)=0$, the restriction of any $\psi_{a}$ to $\Delta_{K}$ is automatically coboundary. For the remaining condition on $\psi$, we compute

$$
\begin{aligned}
& \psi_{a}\left(\left(\frac{n}{m^{\prime}} i_{1}, 0\right),\left(0, \frac{n}{m^{\prime}} j_{2}\right)\right)=\psi_{a}\left(g_{1}^{i_{1}}, g_{1}^{-j_{1}} g_{2}^{m j_{2} / m^{\prime}}\right)=\exp \left(2 \pi i a m i_{1} j_{2} /\left(m^{\prime}\right)^{2}\right) \\
& \psi_{a}\left(\left(0, \frac{n}{m^{\prime}} j_{2}\right),\left(\frac{n}{m^{\prime}} i_{1}, 0\right)\right)=\psi_{a}\left(g_{1}^{-j_{1}} g_{2}^{m j_{2} / m^{\prime}}, g_{1}^{i_{1}}\right)=1
\end{aligned}
$$

These must be equal for all $i_{1}, j_{2}$, so we obtain $\left(m^{\prime}\right)^{2} \mid a m$.

\subsection{Proof of Corollary 2 and other things}

Claim 2. Let $H=\Delta_{K}(1 \times N)$.

(a) Each class in $Z^{2}(H ; \mathbb{T})$ contains a cocycle $\psi$ satisfying

$$
\psi((g n, g),(k m, k))=\beta_{(k, k)}^{\psi}(n, 1)^{*} \psi\left(\left(n^{k}, 1\right),(m, 1)\right) \psi((g, g),(k, k)) .
$$

(b) Conversely, suppose $\psi^{\prime} \in Z^{2}(1 \times N ; \mathbb{T}), \psi \in Z^{2}\left(\Delta_{K} ; \mathbb{T}\right)$, and $\beta_{(g, g)}(n, 1) \in \mathbb{T}$ is a function on $g \in K, n \in N$, satisfying (2.8): i.e. $\beta_{(g k, g k)}(n, 1)=\beta_{(g, g)}(n, 1) \beta_{(k, k)}\left(n^{g}, 1\right)$ for all $g, k \in K, n \in N$. Extend $\psi^{\prime}$ to $H \times H$ by (4.14); then $\psi^{\prime} \in Z^{2}(H ; \mathbb{T})$.

Proof. Consider first part (a). Let $\psi^{\prime} \in Z^{2}(H ; \mathbb{T})$ be arbitrary. Define all $f(k, k)=1$, choose values $f(n, 1)$ arbitrarily, and define $f(k n, k)=\psi^{\prime}((k, k),(n, 1))^{*} f(n, 1)$. Then $\psi:=\psi^{\prime} \delta f^{*}$ is a cocycle cohomologous to $\psi^{\prime}$. We compute $\psi((g, g),(m, 1))=1$, so (2.7) collapses to $\psi((n, 1),(k, k))=\beta_{(k, k)}^{\psi}(n, 1)^{*}$ and the cocycle condition (2.6) tells us $\psi((g, g),(k m, k))=\psi((g, g),(k, k))$. Using this and (2.6),(2.9) we get

$$
\begin{gathered}
\psi((n, 1),(k m, k))=\psi\left((n, 1),\left({ }^{k} m, 1\right)\right) \psi\left(\left(n^{k} m, 1\right),(k, k)\right) \psi\left(\left({ }^{k} m, 1\right),(k, k)\right)^{*} \\
=\frac{\psi\left((n, 1),\left({ }^{k} m, 1\right)\right)}{\beta_{(k, k)}^{\psi}\left({ }^{k} m, 1\right)^{*}}\left(\frac{\beta_{(k, k)}^{\psi}(n, 1) \beta_{(k, k)}^{\psi}\left({ }^{k} m, 1\right) \psi\left((n, 1),\left({ }^{k} m, 1\right)\right)}{\psi\left(\left(n^{k}, 1\right),(m, 1)\right)}\right)^{*}
\end{gathered}
$$

which collapses to agree with (4.14). Using this and the cocycle condition, one finds (4.14) holds in general. Part (b) is also straightforward. QED to Claim 2

Suppose $(H, \psi)$ is type 1 (so $H=\Delta_{K}(1 \times N)$ ), and has trivial left and right centres $\mathcal{C}_{l}(A)=\mathcal{C}_{r}(A)=\operatorname{Vec}_{\mathbb{C}}$. Theorem 4.5 of [53] tells us that the global dimension of the MTC of local modules equals the global dimension of $\mathcal{D}_{\omega}(G)$, namely $|G|^{2}$, 
divided by the square of the quantum-dimension of $A$, namely $|N||G / K|$, and so equals $(|K| /|N|)^{2}$. But $\operatorname{Vec}_{\mathbb{C}}$ has dimension 1 , which forces $N=K$. Assume $\psi$ is put in the form (4.14), which we can rewrite as

$$
\psi((g n, g),(k m, k))=\psi((n, 1),(k, k)) \psi^{\prime}\left(n^{k}, m\right) \psi((g, g),(k, k)),
$$

for some $\psi^{\prime} \in Z^{2}(K ; \mathbb{T})$. The condition $\psi(K, H)=1$ is equivalent to the requirement that $\psi((g, g),(k, k))=1$ for all $g, k \in K$. The requirement on $\psi\left((n, 1),\left(1, n^{\prime}\right)\right)$ is equivalent to the condition that $\psi((n, 1),(k, k))=\psi^{\prime}\left(k^{-1}, n\right) \psi^{\prime}\left(n^{k}, k^{-1}\right)^{*}$. We recover the form for $\psi$ given in Corollary 2 . We also require that the class $\left[\left.\widetilde{\omega}\right|_{H}\right]$ be trivial. But the cocycle $\omega\left(k_{1}, k_{2}, k_{3}\right) \omega\left(k_{1}^{\prime}, k_{2}^{\prime}, k_{3}^{\prime}\right)^{*}$ is coboundary on $G^{2}$ iff $\omega$ is coboundary on $G$. This proves one direction of Corollary 2 .

For the other direction, let $\psi^{\prime} \in Z^{2}(K ; \mathbb{T})$. Define $\psi(K, K)=1$ and $\psi((n, 1),(k, k))$ by the formula given in the previous paragraph. Then according to Claim 2(b), $\psi$ defined as in Corollary 2 is a 2-cocycle of $H=K^{2}$, provided $\beta_{(g k, g k)}(n, 1)=\beta_{(g, g)}(n, 1) \beta_{(k, k)}\left(n^{g}, 1\right)$, i.e. provided

$$
\psi^{\prime}\left(k^{-1} g^{-1}, n\right) \psi^{\prime}\left(n^{g}, g^{-1}\right) \psi^{\prime}\left(n^{g k}, k^{-1}\right)=\psi^{\prime}\left(n^{g k}, k^{-1} g^{-1}\right) \psi^{\prime}\left(g^{-1}, n\right) \psi^{\prime}\left(k^{-1}, n^{g}\right) .
$$

But this follows from the relations $\psi^{\prime}\left(n^{g k}, k^{-1}\right)=\psi^{\prime}\left(n^{g} k, k^{-1}\right) \psi^{\prime}\left(k^{-1}, n^{g}\right) \psi^{\prime}\left(k^{-1}, n^{g} k\right)^{*}$, $\psi^{\prime}\left(n^{g k}, k^{-1} g^{-1}\right)=\psi^{\prime}\left(n^{g} k, k^{-1} g^{-1}\right) \psi^{\prime}\left(k^{-1}, g^{-1} n\right) \psi^{\prime}\left(k^{-1}, n^{g} k\right)^{*}, \quad \psi^{\prime}\left(n^{g} k, k^{-1} g^{-1}\right)=$ $\psi^{\prime}\left(n^{g} k, k^{-1}\right) \psi^{\prime}\left(n^{g}, g^{-1}\right) \psi^{\prime}\left(k^{-1}, g^{-1}\right)^{*}$, and $\psi^{\prime}\left(k^{-1}, g^{-1} n\right)=\psi^{\prime}\left(k^{-1} g^{-1}, n\right) \psi^{\prime}\left(k^{-1}, g^{-1}\right) \psi^{\prime}\left(g^{-1}, n\right)^{*}$, coming from the cocycle condition (2.3).

We can now conclude the proof of the final sentence of Theorem 1(a): choose $\psi$ to be the cocycle in its cohomology class whose existence is promised by Claim 2(a). We see directly from $(4.14)$ that $\psi\left(\Delta_{G}, H\right)=1$. The condition $\beta_{N \times 1}^{\psi}(1 \times N)=1$, which is equivalent to $\psi\left((n, 1),\left(1, n^{\prime}\right)\right)=\psi\left(\left(1, n^{\prime}\right),(n, 1)\right)$, holds for $\psi$ iff it holds for all cocycles in $[\psi]$.

\subsection{Proof of Theorem 2}

Since $G \leq S_{k},\left(\mathcal{A}^{\otimes k}\right)^{G}$ contains $\left(\mathcal{A}^{\otimes k}\right)^{S_{k}}$, and the 3-cocycle $\omega_{G}$ for $\left(\mathcal{A}^{\otimes k}\right)^{G}$ is the restriction of that for $\left(\mathcal{A}^{\otimes k}\right)^{S_{k}}$. So it suffices to prove Theorem 2 for the full symmetric group $G=S_{k}$.

First, let us recall some generalities about group cohomology (see e.g. [11]). Given an embedding $\iota_{K}: K \hookrightarrow G$, we get restriction $\iota_{K}^{*}: H^{n}(G ; \mathbb{T}) \rightarrow H^{n}(K ; \mathbb{T})$, a group homomorphism. Given any finite group $G$ and prime $p$ dividing its order, it is elementary that restriction $\iota_{P}^{*}$ to a $p$-Sylow subgroup $P$ sees only the $p$-primary part of $H^{n}(G ; \mathbb{T})$ (i.e. the elements annihilated by some power of $p$ ), i.e. it kills the $p^{\prime}$-primary part for any prime $p^{\prime} \neq p$. Less obvious is that transfer (see e.g. Theorem 10.3 in [11]) says that restriction $\iota_{P}^{*}$ of the $p$-primary part of $H^{n}(G ; \mathbb{T})$ to $P$ is injective.

Nakaoka (Theorem 5.8 of [65]) has proved that for any embedding $\iota: S_{m} \hookrightarrow S_{k}$ for $m \leq k$, the map $\iota^{*}$ is surjective, with kernel which is a direct summand. Moreover 
(his Corollary 6.7), for $n<k / 2$ restriction $\iota^{*}: H^{n}\left(S_{k} ; \mathbb{T}\right) \rightarrow H^{n}\left(S_{k-1} ; \mathbb{T}\right)$ is an isomorphism. The first few groups $H^{3}\left(S_{k} ; \mathbb{T}\right)$ are

$$
\mathbb{Z}_{2}, \mathbb{Z}_{6}, \mathbb{Z}_{12} \times \mathbb{Z}_{2}, \mathbb{Z}_{12} \times \mathbb{Z}_{2}, \mathbb{Z}_{12} \times \mathbb{Z}_{2} \times \mathbb{Z}_{2},
$$

for $S_{2}, S_{3}, S_{4}, S_{5}$, resp. $S_{k}$ for $k \geq 6$, where it stabilises.

In particular, for $k \geq 3$ the 3 -torsion subgroup in $H^{3}\left(S_{k} ; \mathbb{T}\right)$ is $\mathbb{Z}_{3}$; combining Nakaoka's result with transfer allows us to identify (via $\left.\iota^{*}\right)$ that 3 -torsion in $H^{3}\left(S_{k} ; \mathbb{T}\right)$ $(k \geq 3)$ with $H^{3}\left(C_{3} ; \mathbb{T}\right)$ where $C_{3}=\langle(123)\rangle \cong \mathbb{Z}_{3}$. We write $\left[\omega_{q}^{(3)}\right] \in H^{3}\left(S_{k} ; \mathbb{T}\right)$ for the unique 3 -torsion class which restricts to $\left[\omega_{q}\right] \in H^{3}\left(C_{3} ; \mathbb{T}\right)$ in $(2.13)$. This is the cocycle appearing in the statement of Theorem 2.

Claim 3. Suppose some class $[\omega] \in H^{3}\left(S_{k} ; \mathbb{T}\right)$ is nontrivial. Then there is either a cyclic subgroup $C \leq S_{k}$ of order $\leq 4$ such that the restriction of $[\omega]$ to $C$ is nontrivial, or $k \geq 6$ and the restriction of $\omega$ to $\langle(12)\rangle \times\langle(34)\rangle \times\langle(56)\rangle \cong \mathbb{Z}_{2} \times \mathbb{Z}_{2} \times \mathbb{Z}_{2}$ is cohomologous to $\omega_{\text {iii }}$ of $(2.15)$.

Proof. Without loss of generality we may assume $k=6$, by stability. Write (noncanonically) $H^{3}\left(S_{6} ; \mathbb{T}\right)=\langle x\rangle \times\langle y\rangle \times\langle z\rangle$ where $x, y, z$ have orders $12,2,2$ respectively.

Suppose first that $[\omega] \in H^{3}\left(S_{k} ; \mathbb{T}\right)$ has order a multiple of 3 . Then by the above discussion it must restrict to an order 3 element of $H^{3}\left(C_{3} ; \mathbb{T}\right)$, where $C_{3}$ is as above.

The 2-primary part of $H^{3}\left(S_{k} ; \mathbb{T}\right)$ is more delicate. Suppose first that $[\omega] \in$ $H^{3}\left(S_{k} ; \mathbb{T}\right)$ has order 4 (so $[\omega]$ is of the form $x^{ \pm 3} y^{i} z^{j}$ ). Then by Nakaoka it must restrict to an order- 4 class in $H^{3}\left(S_{4} ; \mathbb{T}\right)$, hence by transfer to an order-4 class for its Sylow 2-subgroup $\langle(1324),(12)\rangle \cong D_{4}$, the dihedral group of order 8 . Explicit cocycles can be computed e.g. by the homological algebra library HAP in GAP; a cocycle of order 4 in $H^{3}\left(D_{4} ; \mathbb{T}\right) \cong \mathbb{Z}_{4} \times \mathbb{Z}_{2} \times \mathbb{Z}_{2}$ is

$$
\omega_{(4)}\left(a^{l} b^{m}, a^{l^{\prime}} b^{m^{\prime}}, a^{l^{\prime \prime}} b^{m^{\prime \prime}}\right)=\left\{\begin{array}{cc}
e^{\pi i(-1)^{m} l / 2} & \text { if } m^{\prime}=0 \text { and } 2 l^{\prime}+2 l^{\prime \prime}+m^{\prime}+m^{\prime \prime} \geq 8 \\
e^{\pi i(-1)^{m+1} l / 2} & \text { if } m^{\prime}=1 \text { and } 2 l^{\prime \prime}+m^{\prime \prime}>2 l^{\prime}+m^{\prime} \\
1 & \text { otherwise }
\end{array}\right.
$$

where we write $D_{4}=\left\langle a, b \mid a^{4}=b^{2}=(a b)^{2}=1\right\rangle$, so $0 \leq l<4,0 \leq m<2$, etc. We see that the restriction of $\omega_{(4)}$ to $\langle a\rangle \cong \mathbb{Z}_{4}$ (i.e. choosing $m=m^{\prime}=m^{\prime \prime}=0$ ) is $\omega_{1}$ (recall $(2.13)$ ), hence order 4 . The order-4 classes for $D_{4}$ equal $\left[\omega_{(4)}^{ \pm 1}\right]$ times an order-2 class; in all cases it restricts to an order-4 cocycle on $C_{4}=\langle(1234)\rangle \cong \mathbb{Z}_{4}$.

Of course, if $[\omega]=\left[\omega_{(4)}\right]^{2}$, then it is also nontrivial (in fact order 2 ) on $C_{4}$. So the only classes remaining to consider are $[\omega] \in\{y, y z, z\}$.

It is convenient to change scalars $\mathbb{T}$. The short exact sequence $0 \rightarrow \mathbb{Z} \rightarrow \mathbb{R} \rightarrow$ $\mathbb{T} \rightarrow 1$ gives a long exact sequence in cohomology whose connecting homomorphisms $\alpha: H^{n}(G ; \mathbb{T}) \rightarrow H^{n+1}(G ; \mathbb{Z})$ are isomorphisms. The short exact sequence $0 \rightarrow \mathbb{Z} \stackrel{\times 2}{\rightarrow}$ $\mathbb{Z} \stackrel{\pi}{\rightarrow} \mathbb{Z}_{2} \rightarrow 0$, where $\pi$ is reduction $\bmod 2$, gives the long exact sequence

$$
\cdots \rightarrow H^{n}(G ; \mathbb{Z}) \stackrel{\times 2}{\rightarrow} H^{n}(G ; \mathbb{Z}) \stackrel{\pi}{\rightarrow} H^{n}\left(G ; \mathbb{Z}_{2}\right) \stackrel{\beta}{\rightarrow} H^{n+1}(G ; \mathbb{Z}) \rightarrow \cdots
$$


The sequence $0 \rightarrow \mathbb{Z}_{2} \stackrel{\epsilon}{\rightarrow} \mathbb{T} \stackrel{\text { square }}{\rightarrow} \mathbb{T} \rightarrow 1$ where $\epsilon$ identifies $\mathbb{Z}_{2}$ with \pm 1 , gives a long exact sequence

$$
\cdots \rightarrow H^{n}\left(G ; \mathbb{Z}_{2}\right) \stackrel{\epsilon}{\rightarrow} H^{n}(G ; \mathbb{T}) \stackrel{\text { square }}{\rightarrow} H^{n}(G ; \mathbb{T}) \stackrel{\gamma}{\rightarrow} H^{n+1}\left(G ; \mathbb{Z}_{2}\right) \rightarrow \cdots
$$

Finally, $0 \rightarrow \mathbb{Z}_{2} \rightarrow \mathbb{Z}_{4} \stackrel{\times 2}{\rightarrow} \mathbb{Z}_{2} \rightarrow 0$ gives a long exact sequence where the connecting homomorphism is called the Steenrod square $S q^{1}$. All of these connecting maps are essentially the same: more precisely, $S q^{1}=\pi \circ \beta=\gamma \circ \epsilon$. However, $S q^{1}$ is the most accessible: it is a derivation on the (commutative) ring $H^{*}\left(G ; \mathbb{Z}_{2}\right.$ ) (where the product is the cup product), and $S q^{1}(a)=a^{2}$ for any class of degree 1 . In all cases, naturality of the long exact sequence implies that the restrictions of cohomology groups intertwine the connecting maps.

A fairly complete description of the $\mathbb{Z}_{2}$-cohomology of the symmetric groups is provided in Chapter VI of [1]. As rings, we have the isomorphisms $H^{*}\left(S_{2} ; \mathbb{Z}_{2}\right) \cong \mathbb{Z}_{2}\left[\sigma_{1}^{\prime}\right]$, and $H^{*}\left(S_{6} ; \mathbb{Z}_{2}\right) \cong \mathbb{Z}_{2}\left[\sigma_{1}, \sigma_{2}, \sigma_{3}, c_{3}\right] /\left(c_{3}\left(\sigma_{3}+\sigma_{1} \sigma_{2}\right)\right)$, where the subscript indicates the degree of the generators. Restriction from $S_{6}$ to $S_{2} \times S_{2} \times S_{2}=\langle(12),(34),(56)\rangle$ (where $S_{6}$ is the permutation group for $\{1,2,3,4,5,6\}$ ) sends $c_{3}$ to 0 , and $\sigma_{1}, \sigma_{2}, \sigma_{3}$ to the generators of the same name of a polynomial subalgebra $\mathbb{Z}_{2}\left[\sigma_{1}, \sigma_{2}, \sigma_{3}\right]$ of $H^{3}\left(S_{2}^{3} ; \mathbb{Z}_{2}\right) \cong H^{3}\left(S_{2} ; \mathbb{Z}_{2}\right) \otimes_{\mathbb{Z}_{2}} H^{3}\left(S_{2} ; \mathbb{Z}_{2}\right) \otimes_{\mathbb{Z}_{2}} H^{3}\left(S_{2} ; \mathbb{Z}_{2}\right)$. Moreover, $S q^{1}\left(\sigma_{2}\right)=$ $\sigma_{1} \sigma_{2}+\sigma_{3}+c_{3}, S q^{1}\left(\sigma_{3}\right)=\left(c_{3}+\sigma_{3}\right) \sigma_{1}, S q^{1}\left(c_{3}\right)=0$, and as always $S q^{1}\left(\sigma_{1}\right)=\sigma_{1}^{2}$ and $S q^{1}(a b)=S q^{1}(a) b+a S q^{1}(b)$.

Equation (4.16) tells us that $\beta\left(H^{3}\left(S_{6} ; \mathbb{Z}_{2}\right)\right)$ equals the order 2 classes in $H^{4}\left(S_{6} ; \mathbb{Z}\right)$, namely $\operatorname{Span}\{6 \alpha(x), \alpha(y), \alpha(z)\} \cong \mathbb{Z}_{2}^{3}$, and that $\pi$ kills $6 \alpha(x)$. Therefore we can identify (through $\beta$ ) the image of $S q^{1}=\pi \circ \beta$ on $H^{3}\left(S_{6} ; \mathbb{Z}_{2}\right)$ with the space $\operatorname{Span}\{\alpha(y), \alpha(z)\} \cong \mathbb{Z}_{2}^{2}$. We compute this image to be $\operatorname{Span}\left\{S q^{1}\left(\sigma_{1}^{3}\right), S q^{1}\left(\sigma_{3}\right)\right\}$. This means we can identify $y=\epsilon\left(\sigma_{1}^{3}\right)$ and $z=\epsilon\left(\sigma_{3}\right)$.

Restriction to $S_{2}^{3}$ of $\sigma_{1}^{3}$ and $\sigma_{3}$ (hence of $y, z$ ) is now straightforward, and we see that $z$ restricts to 0 on $\langle(12)\rangle=: C_{2}$, while $y$ (hence $y z$ ) restricts to $\epsilon\left(\sigma_{1}^{\prime 3}\right) \neq 0$ on $C_{2}$. Using the interpretation of the image of restriction in terms of symmetric invariants, as discussed in section III.4 of [1] $-S_{3}$ acts by permuting the three $S_{2}$ 's - we identify the restriction of $z$ with the 3 -cocycle $\omega_{i i i}$, as desired. QED to Claim 3

So it suffices to consider permutation orbifolds of the form $\left(\mathcal{A}^{\otimes k}\right)^{C}$, where $C$ is a cyclic group generated by an order $n$ cycle $\pi$ in $S_{k}$, where $n=2,3,4$, as well as $\left(\mathcal{A}^{\otimes 6}\right)^{S_{2} \times S_{2} \times S_{2}}$.

We can read off the conformal weights of any simple module in $\left(\mathcal{A}^{\otimes n}\right)^{\langle\pi\rangle}$ from the work $[10,3,4,57,48]$ on permutation orbifolds (or the (3.7) paragraph). For example, [57], Theorem 6.3e gives the conformal weights of the simple summands of the restriction to that permutation orbifold of the unique $\pi$-twisted module of the conformal net $\mathcal{A}^{\otimes n}$ : these are $\frac{j}{n}+\frac{n^{2}-1}{24 n} c$ where $c \in 8 \mathbb{Z}_{\geq 0}$ is the central charge of $\mathcal{A}$ and $0 \leq j<n$. (We thank Marcel Bischoff for correspondence on this point.) Hence the conformal weights of the permutation orbifold with $G \cong \mathbb{Z}_{n}$ for $n=2$, 4 lie in $\frac{1}{n} \mathbb{Z}$. As was discussed at the end of section 2.2, this means that the twist $\omega$ occurring in those $n=2,4$ orbifolds is trivial. However, when $n=3$, the conformal weights lie in $\frac{1}{9} \mathbb{Z}$; 
in particular $c / 9(\bmod 1)$ is a conformal weight. Again from the calculations at the end of section 2.2 , we see this requires the specific twist $\omega=\omega_{c}(\bmod 3) \in H^{3}\left(\mathbb{Z}_{3} ; \mathbb{T}\right)$.

Putting this together with transfer and the results of Nakaoka, we identify the twist $[\omega]$ of the permutation orbifold $\left(\mathcal{A}^{\otimes k}\right)^{G}$ as the restriction to $H^{3}(G ; \mathbb{T})$ of the class we call $\left[\omega_{c(\bmod 3)}^{(3)}\right]$.

Let's turn to $\left(\mathcal{A}^{\otimes 6}\right)^{S_{2} \times S_{2} \times S_{2}}$, where $S_{2} \times S_{2} \times S_{2}=\langle(12)\rangle \times\langle(34)\rangle \times\langle(56)\rangle$. This clearly equals $\left((\mathcal{A} \otimes \mathcal{A})^{S_{2}}\right)^{\otimes 3}$. But we've learned the 3-cocycle for $(\mathcal{A} \otimes \mathcal{A})^{S_{2}}$ will be trivial, so so will that for the $S_{2} \times S_{2} \times S_{2}$ orbifold.

This concludes the proof of Theorem 2 .

\subsection{Proof of Theorems 3 and 4}

Fix any finite group $G$ and normal subgroup $N$. Jones (see section 1.2 of [47]) defines a group $\Lambda(G, N)$, his characteristic invariant, as follows. If the pairs $(\lambda, \mu)$ and $\left(\lambda^{\prime}, \mu^{\prime}\right)$ both satisfy (4.9)-(4.12), then so does their product $\left(\lambda \lambda^{\prime}, \mu \mu^{\prime}\right)$. Let $Z$ denote the resulting (abelian) group of solutions $\lambda: G \times N \rightarrow \mathbb{T}, \mu \in Z^{2}(G ; \mathbb{T})$ to those equations. For any function $\eta: N \rightarrow \mathbb{T}$ with $\eta(1)=1$, and define $\lambda_{\eta}(g, n)=\eta(n) \eta\left(n^{g}\right)^{*}$ and $\mu_{\eta}\left(n, n^{\prime}\right)=\eta\left(n n^{\prime}\right) \eta(n)^{*} \eta\left(n^{\prime}\right)^{*}$. Then $\lambda_{\eta}, \mu_{\eta}$ are readily seen to satisfy (4.9)-(4.12), and so the set of all of them form a subgroup of $Z$. Jones' group $\Lambda(G, N)$ is defined to be the quotient of $Z$ by that subgroup.

Likewise, let $Z^{\prime}$ be the set of all $\psi \in Z^{2}(H ; \mathbb{T})$ satisfying both $\left.[\psi]\right|_{\Delta_{G}}=[1]$ and $\beta_{N \times 1}(1 \times N)=1$ (the conditions on a type $1 \psi$ in Theorem $\left.1(\mathrm{a})\right)$. Since $\beta^{\psi \psi^{\prime}}=\beta^{\psi} \beta^{\psi^{\prime}}$, $Z^{\prime}$ is a subgroup of $Z^{2}(H ; \mathbb{T})$. Since $(n, 1)$ and $\left(1, n^{\prime}\right)$ commute, $\beta_{(n, 1)}^{\psi}\left(1, n^{\prime}\right)=1$ when $\psi$ is coboundary, so $Z^{\prime}$ contains all 2-coboundaries. Define $\mathcal{G}(G, N)$ to be the quotient of $Z^{\prime}$ by all 2-coboundaries; it is the subgroup of $H^{2}(H ; \mathbb{T})$ consisting of all classes $[\psi]$ where $(H, \psi)$ is type 1 .

Claim 4. The map sending $(\lambda, \mu)$ to $\psi\left((g n, g),\left(k n^{\prime}, k\right)\right):=\mu\left(n^{k}, n^{\prime}\right) / \lambda(k, n)$ defines an isomorphism between the groups $\Lambda=\Lambda(G, N)$ and $\mathcal{G}=\mathcal{G}(G, N)$, with inverse sending $\psi$ to $\lambda(g, n)=\beta_{(g, g)}^{\psi}(n, 1)$ and $\mu\left(n, n^{\prime}\right)=\psi\left((n, 1),\left(n^{\prime}, 1\right)\right)$.

Proof. Call a 2-cocycle $\psi \in Z^{2}(H ; \mathbb{T})$ well-chosen if it satisfies $\psi\left(\Delta_{G}, H\right)=1$ and $\beta_{N \times 1}^{\psi}(1 \times N)=1$. Let $(H, \psi)$ be type 1 . Then Theorem $1($ a) says $\psi$ is cohomologous to a well-chosen one. Suppose $\psi$ and $\psi^{\prime}$ are both well-chosen and cohomologous, and write $\psi^{\prime}=\psi \delta f$ for some $f: H \rightarrow \mathbb{T}$ with $f(1,1)=$ 1. Because $\left.\psi\right|_{\Delta_{G}}=\left.\psi^{\prime}\right|_{\Delta_{G}}$, we know $f(g k, g k)=f(g, g) f(k, k)$. Because $\psi((g, g),(n, 1))=\psi^{\prime}((g, g),(n, 1))$, we know $f(g n, g)=f(g, g) f(n, 1)$. We compute $(\delta f)((g n, g),(k m, k))=f\left(n^{k} m, 1\right) f(n, 1)^{*} f(m, 1)^{*}$. Therefore $\psi, \psi^{\prime}$ are both well-chosen and cohomologous iff there is a function $\eta: N \rightarrow \mathbb{T}$ with $\eta(1)=1$ and $\psi^{\prime}((g n, g),(k m, k)) \psi((g n, g),(k m, k))^{*}=\eta\left(n^{k} m\right) \eta(n)^{*} \eta(m)^{*}=: \psi_{\eta}((g n, g),(k m, k))$. We've shown $\mathcal{G}$ is the group $Z^{\prime \prime}$ of all well-chosen $\psi$, quotient the subgroup of all $\psi_{\eta}$.

Because $\mu \in Z^{2}(N ; \mathbb{T})$ and $\lambda$ satisfies (4.10), Claim 2(b) tells us $\psi((g n, g),(k m, k)):=$ $\mu\left(n^{k}, m\right) / \lambda(k, n)$ lies in $Z^{2}(H ; \mathbb{T})$. The resulting $\psi$ is well-chosen: $\psi\left(\Delta_{G}, H\right)=1$ by (4.12), and $\beta_{(n, 1)}^{\psi}\left(1, n^{\prime}\right)=1$ by (4.9) and (4.10). Moreover, $\left(\lambda_{\eta}, \mu_{\eta}\right) \mapsto \psi_{\eta}$, so passing to the quotients, we get a group homomorphism $F: \Lambda \rightarrow \mathcal{G}$. 
For $\psi$ well-chosen, define $\lambda(g, n)=\beta_{(g, g)}^{\psi}(n, 1)=\psi((n, 1),(g, g))^{*}$ and $\mu\left(n, n^{\prime}\right)=$ $\psi\left((n, 1),\left(n^{\prime}, 1\right)\right)$. As mentioned in section $4.5,(\lambda, \mu)$ lie in $Z$. This map is the inverse of the map $Z \rightarrow Z^{\prime \prime}$ of the previous paragraph. The map sends $\psi_{\eta}$ to $\left(\lambda_{\eta}, \mu_{\eta}\right)$, so passing to the quotients we get the inverse $F^{-1}: \mathcal{G} \rightarrow \Lambda$. QED to Claim 4

By an extension of $G$ by $N$, we mean a group $\widetilde{G}$ with a normal subgroup $\widetilde{N} \cong N$ for which $\widetilde{G} / \widetilde{N} \cong G$. Associated to the projection $p: \widetilde{G} \rightarrow G$ is inflation $p^{*}$ : $H^{*}(G ; \mathbb{T}) \rightarrow H^{*}(\widetilde{G} ; \mathbb{T})$. Associated to the embedding $\iota: \widetilde{N} \hookrightarrow \widetilde{G}$ is restriction $\iota^{*}: H^{*}(\widetilde{G} ; \mathbb{T}) \rightarrow H^{*}(\widetilde{N} ; \mathbb{T})$.

Jones (amongst others) showed (Proposition 4.2.5 in [47]) that the standard 5term restriction-inflation exact sequence for finite group extensions $1 \rightarrow \widetilde{N} \rightarrow \widetilde{G} \rightarrow$ $G \rightarrow 1$ can be extended to the right:

$$
\begin{aligned}
& 1 \rightarrow H^{1}(G ; \mathbb{T}) \stackrel{p^{*}}{\rightarrow} H^{1}(\widetilde{G} ; \mathbb{T}) \stackrel{\iota^{*}}{\rightarrow} H^{1}(\widetilde{N} ; \mathbb{T})^{G} \rightarrow H^{2}(G ; \mathbb{T}) \stackrel{p^{*}}{\rightarrow} H^{2}(\widetilde{G} ; \mathbb{T}) \\
& \stackrel{\iota^{*}}{\rightarrow} \Lambda(G, N) \stackrel{\stackrel{\gamma}{\rightarrow}}{\rightarrow} H^{3}(G ; \mathbb{T}) \stackrel{p^{*}}{\rightarrow} H^{3}(\widetilde{G} ; \mathbb{T})
\end{aligned}
$$

In fact this sequence can be continued indefinitely [39], but we only need those next three terms. The map $\gamma$ here coincides with the assignment (4.8); by Claim 4 we can replace $\Lambda$ with $\mathcal{G}$. The map $p^{*}$ there is inflation.

It is well-known that a projective representation of a finite group $G$ can be lifted to a true (i.e. linear) representation of a finite extension of $G$. The cohomological fact underlying this is that there is an extension $\widetilde{G}$ of $G$ (by the Schur multiplier) such that inflation $H^{2}(G ; \mathbb{T}) \rightarrow H^{2}(\widetilde{G} ; \mathbb{T})$ is the 0 map. We need the $H^{3}$ analogue, which is Lemma 7.1.2 in [47]. In fact this holds more generally:

Proposition 3. [67] Let $G$ be finite, and $q \geq 2$. Then there exists an extension $\widetilde{G}$ by a finite abelian group (depending on $q$ ) such that inflation $H^{q}(G ; \mathbb{T}) \rightarrow H^{q}(\widetilde{G} ; \mathbb{T})$ is the 0 map.

Now turn to the proof of Theorem 3. Choose any finite group $G$ and any 3-cocycle $\omega \in Z^{3}(G ; \mathbb{T})$, and define $\widetilde{\omega} \in Z^{3}\left(G^{2} ; \mathbb{T}\right)$ by $(2.21)$. Choose an extension $\widetilde{G}$ of $G$ by some group $N$ so that inflation $p^{*}: H^{3}(G ; \mathbb{T}) \rightarrow H^{3}(\widetilde{G} ; \mathbb{T})$ is trivial. Then exactness of (4.18) says $\gamma$ must be surjective. This means we can choose a type $1 \psi \in Z^{\prime}$ so that $\bar{\omega}^{\psi} \in H^{3}(G ; \mathbb{T})$ is cohomologous with $\omega$.

Fix any holomorphic conformal net $\mathcal{A}$ in central charge $24-$ e.g. the Monstrous moonshine module. Use Cayley's Theorem to fix some embedding of $\widetilde{G}$ in some symmetric group $S_{k}$. Next, perform the permutation orbifold $\left(\mathcal{A}^{\otimes k}\right)^{\tilde{G}}$. By Theorem 2, this has category of representations $\mathcal{D}_{1}(\widetilde{G})$. Then for $N$ and $\psi$ chosen as in the previous paragraph, we know $\left(\Delta_{\tilde{G}}(1 \times N), \psi\right)$ is type 1 . Let $\mathcal{A}^{\prime}$ denote the corresponding extension. Then by Theorem $1, \mathcal{A}^{\prime}$ will be a completely rational conformal net with category of representations $\operatorname{Rep}\left(\mathcal{A}^{\prime}\right)$ braided tensor equivalent to $\mathcal{D}_{\omega}(G)$.

Of course, the extension $\widetilde{G}$ in Proposition 3 is solvable iff $G$ is, since $N$ is abelian here. Since Conjecture 1 is known to hold for any solvable $G$, we get unconditionally that any $\mathcal{D}_{\omega}(G)$ with $G$ solvable, is realised as the $\operatorname{MTC} \operatorname{Mod}(\mathcal{V})$ of some completely rational VOA $\mathcal{V}$. 
After we completed this proof and shared it with Victor Ostrik, he later communicated an alternate proof of Theorem 3, which assumes our Theorem 2, which we now sketch. First, upgrade the homomorphism $K \rightarrow G$ to a tensor functor $F: \operatorname{Vec}(K) \rightarrow \operatorname{Vec}_{\omega}(G)$. Let $I$ be the right adjoint of $F$ and let $A=I(1)$. Then $A$ has a natural lift to the untwisted double of $K$, where it is an étale algebra. Moreover the category $\operatorname{Rep}_{\operatorname{Vec}(K)}(A)$ is tensor equivalent to $\operatorname{Vec}_{\omega}(G)$. The category of local $A$-modules is the centre of $\operatorname{Rep}_{\operatorname{Vec}(K)}(A)$, giving the desired result.

Before we turn to the proof of Theorem 4, let us make some general comments on orbifolds of holomorphic VOAs. Suppose $G$ is a finite solvable group, and $\mathcal{V}$ is a holomorphic VOA on which $G$ acts faithfully. Then $\mathcal{V}^{G}$ is completely rational, and $\operatorname{Mod}\left(\mathcal{V}^{G}\right)$ is a modular tensor category $\mathcal{C}$. This is the situation studied in sections 4 and 5 of [52], and we begin by reviewing what is obtained there. Let $A$ be the commutative algebra in $\mathcal{C}$ capturing the extension $\mathcal{V}^{G} \subset \mathcal{V}$. Then the isomorphism classes of simple objects (the so-called twisted modules) in the full system $\operatorname{Mod}_{\mathcal{C}}(A)$ are in natural bijection with $g \in G$. Choose a representative $X_{g}$ for each $g$. Then for each $g, h \in G$ there are isomorphisms $\mu_{g, h} \in \operatorname{Hom}\left(X_{g} \otimes_{A} X_{h}, X_{g h}\right) \in \mathbb{C}\left(\otimes_{A}\right.$ denotes the tensor product in the fusion category $\left.\operatorname{Mod}_{\mathcal{C}}(A)\right)$, and these will be unique up to nonzero constants. Computing morphisms in $\operatorname{Hom}\left(X_{g} \otimes_{A} X_{h} \otimes_{A} X_{k}, X_{g h k}\right) \cong \mathbb{C}$ by introducing brackets in two ways, we can define $\omega(g, h, k) \in \mathbb{C}^{\times}$by $\mu_{g, h k} \circ\left(1 \otimes_{A} \mu_{h, k}\right)=$ $\omega(g, h, k) \mu_{g h, k} \circ\left(\mu_{g, h} \otimes_{A} 1\right)$. Then $\omega$ obeys the 3 -cocycle condition, and defines a unique class in $H^{3}\left(G ; \mathbb{C}^{\times}\right) \cong H^{3}(G ; \mathbb{T})$ (cohomologous 3 -cocycles can be obtained from $\omega$ by rescaling the $\left.\mu_{g, h}\right)$. We would expect $\mathcal{C}$ to be tensor equivalent to $\mathcal{D}_{\omega}(G)$, but this is not yet proven; however, the main theorem of [52] says that if $\omega$ is coboundary, then $\mathcal{C} \cong \mathcal{D}_{1}(G)$.

Now suppose $\mathcal{V}^{\prime}$ is another holomorphic VOA carrying a faithful action of $G$, and let $\omega^{\prime}$ be its 3-cocycle. Consider the diagonal action of $G$ on the holomorphic VOA $\mathcal{V} \otimes \mathcal{V}^{\prime}$. Then $\mathcal{V} \otimes \mathcal{V}^{\prime} \supset\left(\mathcal{V} \otimes \mathcal{V}^{\prime}\right)^{G} \supset \mathcal{V}^{G} \otimes \mathcal{V}^{G}$. The $(g, h)$-twisted module will be $X_{g} \bowtie X_{h}^{\prime}$, so the $g$-twisted module for the diagonal action will be $X_{g} \bowtie X_{g}^{\prime}$, and the 3 -cocycle will be $\omega_{g, h, k} \omega_{g, h, k}^{\prime}$. This implies that if $[\omega]$ has order $k$ say in $H^{3}(G ; \mathbb{T})$, then the orbifold for the diagonal action of $G$ on $\mathcal{V} \otimes \cdots \otimes \mathcal{V}(k$ times) will have trivial 3 -cocycle, hence the category of modules of the corresponding orbifold will be tensor equivalent to $\mathcal{D}_{1}(G)$.

Now we turn to the proof of Theorem 4 . Let $G$ be any solvable group, and choose any $\omega \in Z^{3}(G ; \mathbb{T})$. Let $\widetilde{G}$ be as in the proof of Theorem 3 given earlier this subsection. As it is an extension of $G$ by an abelian group, it too is solvable.

Choose any even self-dual positive-definite lattice $L$. Then the VOA $\mathcal{V}(L)$ associated to $L$ will be holomorphic. Consider the permutation orbifold of $\mathcal{V}(L)$ by $\widetilde{G}$. The aforementioned papers on permutation orbifolds all show that the twisted sectors $X_{g}$ $(g \neq 1)$ have conformal weight $>0$. Now, every simple module of $\left(\mathcal{V}(L)^{\otimes_{n}}\right)^{\tilde{G}}$ lies in some $X_{g}$, so we see that they all have conformal weights $\geq 0$, and the only ones with conformal weight $=0$ are in $X_{1}$. But $X_{1}=\mathcal{V}(L)^{\otimes_{n}}$, so the only simple module in $X_{1}$ with conformal weight 0 can be $\left(\mathcal{V}(L)^{\otimes_{n}}\right)^{\tilde{G}}$ itself. Thus condition $(*)$ of section 3.3 is satisfied by $\left(\mathcal{V}(L)^{\otimes_{n}}\right)^{\tilde{G}}$. 
Let $N \in \mathbb{Z}_{>0}$ be such that the diagonal action of $\widetilde{G}$ on $\left(\mathcal{V}(L)^{\otimes n}\right)^{\otimes k}$ has trivial 3cocycle. Then $\left(\mathcal{V}(L)^{\otimes n}\right)^{\otimes k}$ is a holomorphic VOA, and $\left(\left(\mathcal{V}(L)^{\otimes n}\right)^{\otimes k}\right)^{\Delta_{\tilde{G}}}$ has category of modules tensor equivalent to $\mathcal{D}_{1}(\widetilde{G})$. Moreover, $\left(\left(\mathcal{V}(L)^{\otimes n}\right)^{\otimes k}\right)^{\Delta_{\tilde{G}}}$ satisfies condition $(*)$, because $\left(\mathcal{V}(L)^{\otimes n}\right)^{\tilde{G}}$ does.

The remainder of the proof of Theorem 4 is exactly as that of Theorem 3, with $\left(\left(\mathcal{V}(L)^{\otimes n}\right)^{\otimes_{k}}\right)^{\Delta_{\tilde{G}}}$ replacing $\left(\mathcal{A}^{\otimes k}\right)^{\tilde{G}}$.

\section{Acknowledgement.}

The authors thank the University of Alberta Mathematics Dept, Cardiff School of Mathematics, University of Warwick Mathematics Institute, Swansea University Dept of Computer Science and the Isaac Newton Institute for Mathematical Sciences, Cambridge during the programme Operator Algebras: Subfactors and their applications, for generous hospitality while researching this paper. They also benefitted greatly from Research-in-Pairs held at Oberwolfach and BIRS, and the von Neumann algebra trimester at the Hausdorff Institute. DEE thanks WWU Münster and Wilhelm Winter whilst TG thanks Karlstads Universitet and Jürgen Fuchs for stimulating environments while part of this paper was written. We also thank Marcel Bischoff, Michael Müger and Victor Ostrik for comments. Their research was supported in part by EPSRC grant nos EP/K032208/1 and EP/N022432/1, PIMS, NSERC and SFB 878.

\section{References}

[1] Adem, A., Milgram, R. J.: Cohomology of Finite Groups. (second edition) Berlin: Springer-Verlag, 2004.

[2] Bantay, P.: Orbifolds, Hopf algebras, and the moonshine. Lett. Math. Phys. 22 (1991), 187-194.

[3] Bantay, P.: Characters and modular properties of permutation orbifolds. Phys. Lett. B419 (1998), 175-178.

[4] Barron, K., Dong, C., Mason, G.: Twisted sectors for tensor product vertex operator algebras associated to permutation groups. Commun. Math. Phys. 227 (2002), 349-384.

[5] Bischoff, M.: Conformal net realizability of Tambara-Yamagami categories and generalized metaplectic modular categories. arXiv://1803.04949.

[6] Böckenhauer, J., Evans, D. E.: Modular invariants, graphs and alpha-induction for nets of subfactors. I. Commun. Math. Phys. 197, 361-386 (1998); II. Commun. Math. Phys. 200, 57-103 (1999); III. Commun. Math. Phys. 205, 183-228 (1999).

[7] Böckenhauer, J., Evans, D. E.: Modular invariants from subfactors: Type I coupling matrices and intermediate subfactors. Commun. Math. Phys. 213, 267-289 (2000).

[8] Böckenhauer, J., Evans, D. E., Kawahigashi, Y.: On $\alpha$-induction, chiral generators and modular invariants for subfactors. Commun. Math. Phys. 208, 429-487 (1999).

[9] Böckenhauer, J., Evans, D. E., Kawahigashi, Y.: Chiral structure of modular invariants for subfactors. Commun. Math. Phys. 210, 733-784 (2000).

[10] Borisov, L., Halpern, M. B., Schweigert, C.: Systematic approach to cyclic orbifolds. Int. J. Mod. Phys. A13 (1998), 125-168.

[11] Brown, K, S.: Cohomology of groups. Graduate Texts in Mathematics, 87. New York: Springer-Verlag, 1994.

[12] Carnahan, S., Miyamoto, M.: Regularity of fixed-point vertex operator subalgebras. (arXiv:1603.05645v3 [math.RT]), 2016.

[13] Carpi, S., Kawahigashi, Y., Longo, R., Weiner, M.: From vertex operator algebras to conformal nets and back. Memoirs Amer. Math. Soc. (to appear); arXiv:1503.01260.

[14] Cheng, C.: A character theory for projective representations of finite groups. Linear Alg. Appl. 469 (2015), $230-242$.

[15] Coste, A., Gannon, T., Ruelle, P.: Finite group modular data. Nucl. Phys. B581 (2000), 679-717.

[16] Creutzig, T., Kanade, S., McRae, R.: Tensor categories for vertex operator superalgebra extensions. arXiv:1705.05017.

[17] Davydov. A.: Modular invariants for group-theoretical modular data, I. J. Alg. 323 (2010), 1321-1348.

[18] Davydov. A.: Bogomolov multiplier, double class-preserving automorphisms, and modular invariants for orbifolds. J. Math. Phys. 55 (2014), 092305.

[19] Davydov. A., Simmons, D.: On Lagrangian algebras in group-theoretical braided fusion categories. J. Alg. 471 (2017), 149-175.

[20] Dijkgraaf, R. Pasquier, V., Roche, P.: Quasi-quantum groups related to orbifold models. Nucl. Phys. B. Proc. Suppl. 18B (1990), 60-72. 
[21] Dijkgraaf, R., Witten, E.: Topological gauge theories and group cohomology. Commun. Math. Phys. 129 (1990), 393-429.

[22] Dong, C., Mason, G.: Quantum Galois theory for compact Lie groups. J. Algebra 214 (1999), 92-102.

[23] Dong, C., Ren, L., Xu, F.: On orbifold theory. Adv. Math. 321 (2017), 1-30.

[24] van Ekeren, J., Möller, S., Scheithauer, N. R.: Construction and classification of holomorphic vertex operator algebras. J. Reine Angew. Math. (to appear); arXiv:1507.08142.

[25] Etingof, P., Gelaki, S., Nikshych, D., Ostrik, V.: Tensor categories. Providence: American Math. Soc., 2015.

[26] Evans, D. E.: Twisted K-theory and modular invariants: I Quantum doubles of finite groups. In: Bratteli, O., Neshveyev, S., Skau, C. (eds.) Operator Algebras: The Abel Symposium 2004. Berlin-Heidelberg: Springer, 2006, pp. 117-144.

[27] Evans, D. E., Gannon, T.: Modular invariants and twisted equivariant K-theory. Commun. Number Th. Phys. 3 (2009), 209-296.

[28] Evans, D. E., Gannon, T.: The exoticness and realisability of twisted Haagerup-Izumi modular data. Commun. Math. Phys. 307 (2011), 463-512.

[29] Evans, D. E., Gannon, T.: Modular invariants and twisted equivariant K-theory II: Dynkin diagram symmetries. J. K-Theory 12 (2013), 273-330.

[30] Evans, D. E., Gannon, T.: Tambara-Yamagami, tori, loop groups and KK-theory (in preparation).

[31] Evans, D. E., Gannon, T.: Modular invariants and KK-theory: finite groups the full story. (work in progress).

[32] Evans, D. E., Pinto, P. T.: Subfactor realisation of modular invariants. Commun. Math. Phys. 237 (2003), 309-363.

[33] Freed, D. S., Hopkins, M. J., Teleman, C.: Twisted equivariant K-theory with complex coefficients. J. Topol. 1 (2008), 16-44.

[34] Freed, D. S., Hopkins, M. J., Teleman, C.: Loop groups and twisted K-theory I. J. Topol. 4 (2011), 737-798.

[35] Fröhlich, J., Fuchs, J., Runkel, I., Schweigert, C.: Correspondences of ribbon categories. Adv. Math. 199 (2006), 192-329.

[36] Fuchs, J., Runkel, I., Schweigert, C.: Conformal correlation functions, Frobenius algebras and triangulations. Nucl. Phys. B624 (2002), 452-468.

[37] Gemünden, T., Keller, C. A.: Orbifolds of lattice vertex operator algebras at $d=48$ and $d=72$. arXiv:1802.10581.

[38] Goff, C., Mason, G., Ng, S.-H.: On the gauge equivalence of twisted quantum doubles of elementary abelian and extra-special 2-groups. J. Algebra 312 (2007), 849-875.

[39] Habegger, N., Jones, V., Pino Ortiz, O., Ratcliffe, J.: Relative cohomology of groups. Comment. Math. Helvetici 59 (1984), 149-164.

[40] Hanaki, A., Miyamoto, M., Tambara, D.: Quantum Galois theory for finite groups. Duke Math. J. 97 (1999), $541-544$.

[41] Huang, H.-L., Wan, Z., Ye, Y.: Explicit cocycle formulas on finite abelian groups with applications to braided linear Gr-categories and Dijkgraaf-Witten invariants; arXiv:1703.03266.

[42] Huang, Y.-Z. Rigidity and modularity of vertex tensor categories. Commun. Contemp. Math. 10 (2008), 871911.

[43] Huang, Y.-Z., Kirillov, A. Jr, Lepowsky, J.: Braided tensor categories and extensions of vertex operator algebras. Commun. Math. Phys. 337 (2015), 1143-1159.

[44] Hughes, N. J. S.: The use of bilinear mappings in the classification of groups of class 2. Proc. AMS 2 (1951), $742-747$.

[45] Johnson-Freyd, T.: The moonshine anomaly. Commun. Math. Phys. (to appear); arXiv: 1707.08388v2.

[46] Jones, V. F. R.: An invariant for group actions. Algèbres d'opérateurs (Sém., Les Plans-sur-Bex, 1978), pp. 237-253, Lecture Notes in Math., 725, Springer, Berlin, 1979.

[47] Jones, V. F. R.: Actions of finite groups on the hyperfinite type $I I_{1}$ factor. Mem. Amer. Math. Soc. 28 (1980), no. 237.

[48] Kac, V. G., Longo, R., Xu, F.: Solitons in affine and permutation orbifolds. Commun. Math. Phys. 253 (2005), 723-764.

[49] Karpilovsky, G.: Group Representations. Vol. 2. North-Holland Mathematics Studies, 177. Amsterdam: NorthHolland Publishing Co., 1993.

[50] Kawahigashi, Y.: Conformal field theory, tensor categories and operator algebras. J. Phys. A 48 (2015) no.30, 303001.

[51] Kawahigashi, Y., Longo, R., Müger, M.: Multi-interval subfactors and modularity of representations in conformal field theory. Commun. Math. Phys. 219 (2001), 631-669.

[52] Kirillov, A. Jr.: Modular categories and orbifold models. Commun. Math. Phys. 229 (2002), 309-335.

[53] Kirillov, A. Jr., Ostrik, V.: On a q-analogue of the McKay correspondence and the ADE classification of $\mathrm{sl}(2)$ conformal field theories. Adv. Math. 171 (2002), 183-227.

[54] Kosaki, H., Munemasa, A., Yamagami, S.: On fusion algebras associated to finite group actions. Pac. J. Math. 177 (1997), 269-290. 
[55] Lepowsky, J., Li, H.: Introduction to Vertex Operator Algebras and Their Representations. (Birkhäuser, Boston 2004).

[56] Longo, R., Rehren, J.-H.: Nets of subfactors. Rev. Math. Phys. 7 (1995), 567-597.

[57] Longo, R., Xu, F.: Topological sectors and a dichotomy in conformal field theory. Commun. Math. Phys. 251 (2004) 321-364.

[58] Majid, S.: Quantum double for quasi-Hopf algebras. Lett. Math. Phys. 45 (1998), 1-9.

[59] Mason, G., Ng, S.-H.: Generalized twisted quantum doubles of a finite group and rational orbifolds. arXiv:1703.06489.

[60] Miyamoto, M.: $C_{2}$-cofiniteness of cyclic-orbifold models. Commun. Math. Phys. 335(3) (2015), 1279-1286.

[61] Miyamoto, M.: C2-cofiniteness of orbifold models for finite groups. arXiv:1812.00570

[62] Müger, M.: Conformal field theory and Doplicher-Roberts reconstruction. In: Mathematical Physics in Mathematics and Physics: Quantum and Operator Algebraic Aspects, R. Longo (ed.), Fields Inst. Commun. 20, pp.297-319 (2001).

[63] Müger, M.: On the structure of braided crossed G-categories. Appendix to V.Turaev, Homotopy quantum field theory, volume 10 of EMS Tracts in Mathematics. European Mathematical Society, Zürich, 2010.

[64] Naidu, D., Nikshych, D.: Lagrangian subcategories and braided tensor equivalences of twisted quantum doubles of finite groups. Commun. Math. Phys. 279 (2008) 845-872.

[65] Nakaoka, M.: Decomposition theorem for homology groups of symmetric groups. Ann. Math. 71 (1960), $16-42$.

[66] Ocneanu, A.: Paths on Coxeter diagrams: From Platonic solids and singularities to minimal models and subfactors. (Notes recorded by S. Goto). In: Rajarama Bhat, B.V. et al. (eds.) Lectures on Operator Theory. Providence: American Mathematical Society, 2000, pp. 243-323.

[67] Opolka, H.: Group extensions and cohomology groups II. J. Alg. 169 (1994), 328-331.

[68] Ostrik, V.: Module categories, weak Hopf algebras and modular invariants. Transform. Groups 8 (2003), $177-206$.

[69] Ostrik, V.: Module categories for quantum doubles of finite groups. Int. Math. Res. Notices 27 (2003), 15071520.

[70] Raeburn, I., Sims, A., Williams, D. P.: Twisted actions and obstructions in group cohomology. In: $C^{*}$-algebras (Münster, 1999), Cuntz J., Echterhoff S. (eds), pp.161-181, Springer, Berlin, 2000.

[71] Tener, J.: Geometric realization of algebraic conformal field theories. arXiv:1611.01176

[72] Xu, F.: New braided endomorphisms from conformal inclusions. Commun. Math. Phys. 192, 349-403 (1998).

[73] Xu, F.: Algebraic orbifold conformal field theories. Proc. Natl. Acad. Sci. USA 97, no. 26 (2000), 14069-14073. 